\title{
Classificação de Singularidades: O Método da Transversal Completa
}

\author{
Lee Yun Sheng
}

Orientadora: Prof ${ }^{a}$. Roberta G. W. Atique

USP - São Carlos

Março de 2002 
Aos meus pais,

Kou Hsien

e

Hui Chu. 


\section{Agradecimentos}

Primeiramente um agradecimento em especial à Profa. Dra. Roberta G. W. Atique, minha orientadora, não só pela dedicação, cuidado e paciência em me orientar mas também pela sua amizade e incentivo nas horas mais adversas.

À minha família pelo apoio e compreensão, e de maneira mais especial aos meus pais que se sacrificaram várias vezes para que seu filho continuasse e chegasse até onde esta.

Aos professores do ICMC que contribuíram com a minha formação e/ou amizade: Ires (por ser uma verdadeira "tia"), Janete, Miriam, Edna, Renata, Dide, Sandra, Valdir, Arraut, Wagner, Hermano, Hermínio, Mirinha, Cidinha, Gaspar, Gutierrez, Eduardo, Alexandre, Marcelo e Farid.

A minha sala de pós-graduação: Luciana (pela amizade, conselhos e presteza), Sílvia (por ser uma irmãzinha), William (por ser uma pessoa mais que divertida...) e Márcio \& Eliane.

Aos funcionários da Biblioteca do ICMC, das seções de pós-graduação e graduação, da área financeira e da diretoria.

Aos meus amigos alunos da USP - São Carlos pela amizade e de maneira especial: Dani, José \& Andréa, Carioquinha, Carlos, Claudemir \& Lu, Liane \& Humberto, Marcelo Buosi, Rubinha, Edsandra, Karina, Érica Filletti, Homero, Aline, Érica Mendonça, Lizandra e Rodolfo. 
Aos meus amigos das Unesp's: Larissa, João Carlos, Gislaine, Karen de Lolo, Fernanda e Valéria.

Aos meus amigos do peito, mesmo estando longe me deram força: Romário \& Paulinha, Miguel \& Marília, Juliana Simões, Vanessa, Regina e em especial a Karen Yared, por ter acompanhado nestes últimos sete anos com a sua amizade e palavras.

Agradeço também ao $\mathrm{CNPq}$ pelo suporte financeiro que possibilitou a realização deste trabalho.

Peço desculpas as pessoas que contribuíram direta ou indiretamente no meu mestrado e não os citei, mas agradeço a todos.

Finalmente peço a Deus que abençoe a todos! Muito obrigado. 


\section{Resumo}

Através do Método da Transversal Completa apresentamos neste trabalho a classificação dos germes simples de $\mathbb{R}^{n} \mathrm{em} \mathbb{R}$, a classificação dos germes do plano no plano de corank 1 e $\mathcal{A}$-codimensão no máximo 4 e uma breve classificação de bigermes de $\mathbb{R}$ em $\mathbb{R}^{2}$. 


\section{Abstract}

Applying the Complete Transversal Method we obtain, in this work, a classification of simple germs of smooth function from $\mathbb{R}^{n}$ to $\mathbb{R}$, a classification of germs of maps from the plane to the plane with $\mathcal{A}$-codimension up to 4 of corank 1 and an introduction to the classification of bigerms of maps from $\mathbb{R}$ to $\mathbb{R}^{2}$. 


\section{Sumário}

$\begin{array}{ll}\text { Prefácio } & 1\end{array}$

1 Introdução 3

1.1 Aplicações de classe $C^{\infty}$ e Variedades . . . . . . . . . . . . 3

1.2 Ação de Grupos . . . . . . . . . . . . . . . . . . . . 7

1.3 A álgebra $\varepsilon_{n} \ldots \ldots \ldots \ldots \ldots \ldots \ldots \ldots$

1.4 Grupo $\mathcal{R} \ldots \ldots \ldots \ldots \ldots$

1.5 Germes Finitamente Determinados . . . . . . . . . . . 12

1.6 Desdobramentos e Deformações . . . . . . . . . . . . . . . 13

2 Classificação de Germes de Funções 15

2.1 Singularidades de corank $\leq 1 \ldots \ldots \ldots \ldots$

2.2 O Método da Transversal Completa . . . . . . . . . . . . . . 19

2.3 Singularidades de Corank $2 \ldots \ldots \ldots \ldots$

2.4 Singularidades Simples . . . . . . . . . . . . . . . . . . . . 29

3 Classificação dos germes $\left(\mathbb{R}^{2}, 0\right) \rightarrow\left(\mathbb{R}^{2}, 0\right)$

$3.1 \quad$ O grupo $\mathcal{A} \ldots \ldots \ldots \ldots \ldots \ldots$

3.2 A Classificação . . . . . . . . . . . . . . . . . . . . 43

4 Classificação de Bigermes de $(\mathbb{R}, S) \rightarrow\left(\mathbb{R}^{2}, 0\right)$

4.1 Resultados Gerais . . . . . . . . . . . . . . . . . 55

4.2 A Classificação . . . . . . . . . . . . . . . . . 57

$\begin{array}{ll}\text { Referências Bibliográficas } & 69\end{array}$ 


\section{Prefácio}

A busca de modelos, ou classificação de objetos, é uma das principais preocupações em matemática. A ferramenta que transforma os objetos em modelos é uma relação de equivalência. Cada área da matemática tem sua noção natural de relação de equivalência e é igualmente natural querer listar os objetos em questão a menos desta equivalência. Assim, por exemplo, temos a noção de isomorfismos para espaços vetoriais ou grupos, difeomorfismos para variedades. A motivação para a busca de uma forma simples para o representante de uma classe de equivalência se deve ao fato de que tal modelo possui todas as propriedades dos elementos de sua classe. Além disso, os cálculos com o modelo são mais simples.

$\mathrm{Na}$ teoria de singularidade de germes de aplicações diferenciáveis, uma noção de equivalência consiste em mudança de coordenadas na fonte e meta.

O germe de uma aplicação $f: \mathbb{R}^{n} \rightarrow \mathbb{R}^{p}$ é singular em 0 se a matriz jacobiana de $f$ em 0 , denotada por $d f_{0}$, não tem rank máximo. Caso contrário, $f$ é regular. Se $f$ é regular em 0: e $n \geq p$ segue da Forma Local das Submersões que existem sistemas de coordenadas em $\mathbb{R}^{n}$ e $\mathbb{R}^{p}$ em relação às quais $f$ é dada por $\left(x_{1}, \ldots, x_{n}\right) \mapsto\left(x_{1}, \ldots, x_{p}\right)$; e $n \leq p$ segue da Forma Local das Imersões que existem sistemas de coordenadas em $\mathbb{R}^{n}$ e $\mathbb{R}^{p}$ em relação aos quais $f$ é dada por $\left(x_{1}, \ldots, x_{n}\right) \mapsto\left(x_{1}, \ldots, x_{n}, 0, \ldots, 0\right)$. No caso de germes de funções (isto é, $p=1$ ), se o gradiente de $f$ for nulo na origem e se a matriz Hessiana de $f$ na origem for invertível (em outras palavras, a origem é um ponto crítico não degenerado), o Lema de Morse (1.5.8) afirma que $f$ é equivalente a $\left(x_{1}, \ldots, x_{n}\right) \mapsto \pm x_{1}^{2} \pm x_{2}^{2} \pm \ldots \pm x_{n}^{2}$.

Ainda no caso $p=1$, se a origem é um ponto crítico degenerado, apresentamos no Capítulo 2 a lista das singularidades simples. Tal lista é obtida através do Método da Transversal Completa que é descrito na Seção 2.2. 
O caso $n=p=2$ é estudado no Capítulo 3 e, através do Método da Transversal Completa, apresentamos a classificação dos germes do plano no plano de $\mathcal{A}$-codimensão $\leq 4$ e de corank 1 . Mais ainda, utilizamos o software "Transversal" [10] para o cálculo de algumas codimensões e transversais completas.

No Capítulo 4 usando novamente o Método da Transversal Completa apresentamos um breve início sobre a classificação de bigermes de $\mathbb{R}$ em $\mathbb{R}^{2}$.

Os resultados básicos da Teoria de Singularidades necessárias para o desenvolvimento destes estudos são apresentados no Capítulo 1. 


\section{Capítulo 1}

\section{Introdução}

Neste capítulo apresentamos resultados da Teoria de Singularidades que serão usados nos capítulos seguintes. A referência principal é [2].

\subsection{Aplicações de classe $C^{\infty}$ e Variedades}

Nesta seção, $U$ é um subconjunto aberto de $\mathbb{R}^{n}$.

Teorema 1.1.1 (da Função Inversa) Sejam $f: U \rightarrow V, V \subset \mathbb{R}^{n}$ aberto, e $x \in U$ tais que $d f_{x}: \mathbb{R}^{n} \rightarrow \mathbb{R}^{n}$ é um isomorfismo. Então existem vizinhanças abertas $U^{\prime}$ e $V^{\prime}$ de $x$ e $f(x)$ respectivamente tais que a restrição $\left.f\right|_{U^{\prime}}: U^{\prime} \rightarrow V^{\prime}$ é um difeomorfismo. Neste caso, dizemos que $f$ é um difeomorfismo local.

Definição 1.1.2 Seja $x \in U$. Dizemos que uma aplicação de classe $C^{\infty}$ $f: U \rightarrow \mathbb{R}^{p}$ é uma imersão em $x$ se $d f_{x}: \mathbb{R}^{n} \rightarrow \mathbb{R}^{p}$ for injetora (notemos que necessariamente $n \leq p)$. Dizemos que $f$ é submersão em $x$ se $d f_{x}: \mathbb{R}^{n} \rightarrow \mathbb{R}^{p}$ for sobrejetora $(n \geq p)$.

Dizemos que $f$ é submersão (respectivamente imersão) se $f$ for submersão (respectivamente imersão) em todo $x \in U$.

Proposição 1.1.3 (Forma Local das Submersões) Seja $f: U \rightarrow \mathbb{R}^{p}$ uma aplicação de classe $C^{\infty}$ tal que $f(0)=0$ e $f$ é uma submersão em 
0. Então existe um difeomorfismo $h: V \rightarrow W, V$ e $W$ vizinhanças de zero em $\mathbb{R}^{n}$, tal que $h(0)=0$ e $\left(f \circ h^{-1}\right)\left(x_{1}, \ldots, x_{n}\right)=\left(x_{1}, \ldots, x_{p}\right)$.

Proposição 1.1.4 (Forma Local das Imersões) Seja $f: U \rightarrow \mathbb{R}^{p}$ uma aplicação de classe $C^{\infty}$ tal que $f(0)=0$ e $f$ é uma imersão em 0. Então existe um difeomorfismo $k: V \rightarrow W, V$ e $W$ vizinhanças de zero em $\mathbb{R}^{p}$, $k(0)=0$ tal que

$$
(k \circ f)\left(x_{1}, \ldots, x_{n}\right)=\left(x_{1}, \ldots, x_{n}, 0, \ldots, 0\right)
$$

Seja $X \subset \mathbb{R}^{n}$ um subconjunto qualquer. Uma vizinhança aberta de $x \in X$ é um conjunto da forma $U \cap X$ onde $x \in U$.

Definição 1.1.5 Sejam $X$ e $Y$ subconjuntos de $\mathbb{R}^{n}$ e $\mathbb{R}^{p}$, respectivamente. Dizemos que $f: X \rightarrow Y$ é uma uma aplicação de classe $C^{\infty}$ se $\forall x \in X$ existir uma vizinhança aberta $U$ de $x$ em $\mathbb{R}^{n}$ e uma aplicação de classe $C^{\infty}$ $F: U \rightarrow \mathbb{R}^{p}$ tal que $F=f$ em $U \cap X$. Dizemos que $f$ é um difeomorfismo se $f$ é bijetora e $f$ e $f^{-1}$ são $C^{\infty}$. Neste caso dizemos que $X$ e $Y$ são difeomorfos.

Definição 1.1.6 Uma parametrização (de dimensão $n$ ) de um conjunto $X \subset$ $\mathbb{R}^{q}$ é uma aplicação de classe $C^{\infty} \phi: V \rightarrow \mathbb{R}^{q}$ onde $\phi: V \rightarrow \phi(V)$ é um difeomorfismo e $\phi(V)=X$.

Dizemos que $N \subset \mathbb{R}^{q}$ é uma variedade diferenciável de dimensão $n$ se para todo $x \in N$ admite uma vizinhança $U \cap N$ e uma parametrização $\phi: V \rightarrow \mathbb{R}^{k}$, $\phi(V)=U \cap N$.

Definição 1.1.7 Seja $N \subset \mathbb{R}^{q}$ uma variedade diferenciável de dimensão $n$. Uma subvariedade diferenciável de $N$ é uma variedade $M \subset \mathbb{R}^{q}$ tal que $M \subset N$. Dizemos que $n-m$ é a codimensão de $M$ em $N$.

Definição 1.1.8 Sejam $N \subset \mathbb{R}^{q}$ uma variedade de dimensão $n, x \in N$ e $\phi: V \rightarrow \mathbb{R}^{q}$ uma parametrização de $N$ tal que $\phi(0)=x$. Definimos o espaço tangente a $N$ em $x$ como sendo $d \phi_{0}\left(\mathbb{R}^{n}\right)$ e o denotamos por $T_{x} N$.

Observemos que $T_{x} N$ está bem definido, isto é, independe da escolha de $\phi$. De fato, seja $\psi: U \rightarrow \mathbb{R}^{k}$ outra parametrização de uma vizinhança de $x \in N, \psi(0)=x$. Podemos supor $\psi(V)=\phi(U)$ (escolhendo $U$ e $V$ se necessário). Seja $h=\psi^{-1} \circ \phi$, temos assim que $d \phi_{0}\left(\mathbb{R}^{n}\right)=d \psi_{0}\left(d h_{0}\left(\mathbb{R}^{n}\right)\right)$, daí 
$d \phi_{0}\left(\mathbb{R}^{n}\right) \subset d \psi_{0}\left(\mathbb{R}^{n}\right)$. Da mesma forma se $g=\phi^{-1} \circ \psi$, obtemos $d \psi_{0}\left(\mathbb{R}^{n}\right) \subset$ $d \phi_{0}\left(\mathbb{R}^{n}\right)$.

Sejam $M \subset \mathbb{R}^{p}$ e $N \subset \mathbb{R}^{q}$ variedades diferenciáveis de dimensões $m$ e $n$ respectivamente, $f: M \rightarrow N$ uma aplicação de classe $C^{\infty}$ e $x \in M$. Seja $y=f(x)$. Então existem $W \subset \mathbb{R}^{p}$ vizinhança aberta de $x$ e $F: W \rightarrow \mathbb{R}^{q}$ uma aplicação de classe $C^{\infty}$ tais que $F=f$ em $W \cap M$.

Proposição 1.1.9 Sob as hipóteses dadas acima temos $d F_{x}\left(T_{x} M\right) \subset T_{y} N$.

\section{Demonstração:}

Sejam $\phi: U \rightarrow \mathbb{R}^{p}$ e $\psi: V \rightarrow \mathbb{R}^{q}$ parametrizações de vizinhanças abertas de $x$ em $M$ e $y$ em $N$ respectivamente, tais que $\phi(u)=x, \psi(v)=y$ e $\phi(U) \subset W$.

Assim $(f \circ \phi)(U) \subset \psi(V)$ (encolhendo $U$ e $V$ se necessário).

Derivando, obtemos $d F_{x}\left(d \phi_{u}\left(\mathbb{R}^{m}\right)\right) \subset d \psi_{v}\left(\mathbb{R}^{n}\right)$. Logo $d F_{x}\left(T_{x} M\right) \subset T_{y} N$.

No que segue $N$ e $P$ são variedades diferenciáveis de dimensão $n$ e $p$, respectivamente.

Definição 1.1.10 Sejam $V$ um espaço vetorial e $U, W \subset V$ subespaços. Dizemos que $U$ e $W$ se intersectam transversalmente se $U+W=V$.

Definição 1.1.11 Sejam $N_{1}$ e $N_{2}$ duas subvariedades de $N$ e $x \in N_{1} \cap N_{2}$. Dizemos que $N_{1}$ e $N_{2}$ se intersectam transversalmente em $x$ quando $T_{x} N_{1}$ e $T_{x} N_{2}$ se intersectam transversalmente em $T_{x} N$. Dizemos que $N_{1}$ e $N_{2}$ se intersectam transversalmente em $N$ quando se intersectam transversalmente em todo $x \in N_{1} \cap N_{2}$.

Definição 1.1.12 Sejam $f: N \rightarrow P$ uma aplicação de classe $C^{\infty}, Q$ uma subvariedade diferenciável de $P$. Definimos o gráfico de $f$ pelo conjunto

$$
\text { graf } f=\{(x, f(x)) / x \in N\}
$$

Dizemos que $f$ é transversal a $Q$ se o gráfico de $f$ e $N \times Q$ se intersectam transversalmente em $N \times P$. 
Proposição 1.1.13 Sejam $f: N \rightarrow P$ uma aplicação de classe $C^{\infty}$ e $Q$ uma subvariedade de $P$. Então $f$ é transversal a $Q$ se, e somente se,

$$
d f_{x}\left(T_{x} N\right)+T_{y} Q=T_{y} P
$$

para todo $x \in N$ tal que $f(x)=y \in Q$.

Proposição 1.1.14 Sejam $f: N \rightarrow P$ uma aplicação de classe $C^{\infty}$ e $Q$ uma subvariedade de $P$ de dimensão $q$, tais que $f$ é transversal a $Q$. Então $M=f^{-1}(Q)$ é uma subvariedade diferenciável de $N$ de mesma codimensão que $Q$, ou vazio. Além disso, se $x \in N$ é tal que $y=f(x) \in Q$, então $T_{x} M=\left(d f_{x}\right)^{-1}\left(T_{y} Q\right)$.

Definição 1.1.15 Sejam $f: N \rightarrow P$ uma aplicação de classe $C^{\infty}$ e $y \in P$. Dizemos que $y$ é valor regular de $f$ se $f$ for transversal a $\{y\}$. Se $x \in N$ é tal que $f(x)$ não é valor regular de $f$ então dizemos que $x$ é ponto crítico de $f$ e $f(x)$ é chamado valor crítico.

Observação 1.1.16 Seja $f$ como na Definição 1.1 .15 e $\operatorname{Im} f$ o conjunto imagem de $f$. Se $y \notin \operatorname{Im} f$, então $y$ é valor regular.

Seja $x \in N$. Consideremos o conjunto das aplicações de classe $C^{\infty}$ definidas numa vizinhança de $x \in N$ com valores em $P$. Neste conjunto vamos introduzir uma relação de equivalência. Sejam $f: U \rightarrow P$ e $g: V \rightarrow P$ neste conjunto. Dizemos que $f$ é equivalente a $g$ se existir uma vizinhança $W$ de $x$ em $N$ tal que $\left.f\right|_{W}=\left.g\right|_{W}$.

Definição 1.1.17 As classes de equivalência são chamadas de germes de aplicações de classe $C^{\infty}$ em $x$.

Notação: $f:(N, x) \rightarrow(P, y)$, onde $y=f(x)$. Dizemos que $x$ é a fonte do germe e $y$ é meta do germe.

Definição 1.1.18 Se $f: N \rightarrow P$ for um difeomorfismo local em $x$, dizemos que a classe de equivalência de $f$ é um germe de difeomorfismo.

Definição 1.1.19 O rank de um germe $f:(N, x) \rightarrow(P, y)$ é o rank de $d f_{x}: T_{x} N \rightarrow T_{y} P$. Se o rank de $f$ for igual à dimensão de $N$ dizemos que $f$ é um germe de uma imersão. Se o rank de $f$ for igual à dimensão de $P$ 
dizemos que $f$ é um germe de uma submersão. Dizemos que $f$ é singular se $\operatorname{rank} f<\min \{\operatorname{dim} N, \operatorname{dim} P\}$.

Uma singularidade de uma aplicação $f: N \rightarrow P$ de classe $C^{\infty}$ é um ponto $x \in N$ tal que o germe de $f$ em $x$ é singular.

Observação 1.1.20 Uma singularidade é um ponto crítico. A recíproca não é verdadeira. Se o germe de $f$ em $x$ é imersivo e $\operatorname{dim} N<\operatorname{dim} P$, então $x$ é ponto crítico mas não é singularidade.

Teorema 1.1.21 (de Sard) Seja $f: N \rightarrow P$ uma aplicação de classe $C^{\infty}$. $O$ conjunto dos valores críticos de $f$ tem medida nula.

Denotamos por $J^{k}(n, p)$ o espaço vetorial real das aplicações $f: \mathbb{R}^{n} \rightarrow \mathbb{R}^{p}$, onde cada componente $f_{i}$ é um polinômio de grau $\leq k$ nas coordenadas $x_{1}, \ldots, x_{n}$ de $\mathbb{R}^{n}$, com termo constante não nulo. Os elementos de $J^{k}(n, p)$ são ditos $k$-jatos. Notemos que $J^{k}(n, p)$ é uma variedade. Seja $f: \mathbb{R}^{n} \rightarrow \mathbb{R}^{p}$ uma aplicação de classe $C^{\infty}$. A série de Taylor de $f(x+a)$ em 0 é dada por

$$
f(a)+d f_{a} x+\frac{1}{2 !} d^{2} f_{a} x^{2}+\frac{1}{3 !} d^{3} f_{a} x^{3}+\ldots
$$

Definição 1.1.22 O $k$-jato de $f$ em $a, k \geq 1, k \in \mathbb{Z}$ é o polinômio

$$
j^{k} f(a)=d f_{a} x+\frac{1}{2 !} d^{2} f_{a} x^{2}+\frac{1}{3 !} d^{3} f_{a} x^{3}+\ldots+\frac{1}{k !} d^{k} f_{a} x^{k}
$$

\subsection{Ação de Grupos}

Sejam $G$ um grupo e $M$ um conjunto. Uma ação de $G$ em $M$ é uma aplicação $\phi: G \times M \rightarrow M$, denotada por $\phi(g, x)=g \cdot x$, satisfazendo:

1. $1 \cdot x=x$, onde 1 é a identidade de $G$

2. $(g h) \cdot x=g \cdot(h \cdot x), \forall x \in M, \forall g, h \in G$

Dada uma ação podemos definir uma relação de equivalência em $M$. Se $x, y \in M, x$ é equivalente a $y$ se existe $g \in G$ tal que $y=g \cdot x$. As classes de equivalência são chamadas órbitas.

Se $x \in M$, a órbita de $x$ é o conjunto

$$
G \cdot x=\{g \cdot x / g \in G\}
$$


Exemplo 1.2.1 Seja $H^{d}(n, p)$ o subespaço de $J^{d}(n, p)$ constituído das aplicações $\mathbb{R}^{n} \rightarrow \mathbb{R}^{p}$ cujas componentes são polinômios homogêneos de grau $d$ nas coordenadas $x_{1}, \ldots, x_{n}$ de $\mathbb{R}^{n}$.

Seja $G L(s)$ o grupo das matrizes $s \times s$ invertíveis (ou o grupo das aplicações lineares $\mathbb{R}^{s} \rightarrow \mathbb{R}^{s}$ invertíveis com a operação de composição).

Uma ação $\phi: G L(n) \times G L(p) \times H^{d}(n, p) \rightarrow H^{d}(n, p)$ do grupo $G L(n) \times$ $G L(p)$ em $H^{d}(n, p)$ é definida da seguinte maneira:

$$
\phi((H, K), f)=(H, K) \cdot f=K \circ f \circ H^{-1}
$$

Definição 1.2.2 Um grupo de Lie G é um grupo que é uma variedade diferenciável e as aplicações de multiplicação $G \times G \rightarrow G$, dada por $\left(g_{1}, g_{2}\right) \mapsto$ $g_{1} g_{2}$, e inversão $G \rightarrow G$, dada por $g \mapsto g^{-1}$, são de classe $C^{\infty}$.

Proposição 1.2.3 Seja $\phi: G \times M \rightarrow M$ uma ação de um grupo de Lie $G$ numa variedade $M$. Suponhamos que as órbitas são subvariedades de $M$. Se $x \in M$, então $\phi_{x}: G \rightarrow G \cdot x$ dada por $\phi_{x}(g)=g \cdot x$ é uma submersão.

Corolário 1.2.4 Com as hipóteses da Proposição 1.2.3 temos que $T_{x} G \cdot x=$ $d \phi_{x_{1}}\left(T_{1} G\right)\left(=d \phi_{x}(1)\left(T_{1} G\right)\right)$.

Exemplo 1.2.5 Sejam $G=G L(n) \times G L(p), M=H^{d}(n, p)$ e $F \in H^{d}(n, p)$, denotamos $F=\left(f_{1}, \ldots, f_{p}\right)$. Definimos

$$
T_{F} G \cdot F=\left\langle\frac{\partial F}{\partial x_{i}} x_{j}\right\rangle_{i, j=1, \ldots, n}+Q
$$

onde $Q$ é o subespaço gerado pelos elementos $g=\left(g_{1}, \ldots, g_{p}\right)$, em que $g_{i}=f_{j}$ e os demais $g_{i}^{\prime} s$ são nulos, $i, j=1, \ldots, p$. Se $p=1$,

$$
T_{F} G \cdot F=\left\langle\frac{\partial F}{\partial x_{i}} x_{j}\right\rangle_{i, j=1, \ldots, n}
$$

Lema 1.2.6 (de Mather) Seja $G$ um grupo de Lie agindo numa variedade M. Seja $N \subset M$ uma subvariedade conexa e suponhamos que as órbitas são subvariedades de $M$. Então $N$ está contida numa única órbita se, e somente se,

1. $T_{y} G \cdot y \supset T_{y} N, \forall y \in N$

2. $\operatorname{dim} T_{y} G \cdot y=$ constante, $\forall y \in N$. 


\subsection{A álgebra $\varepsilon_{n}$}

Sejam $N$ uma variedade e $x \in N$. Estamos interessados em germes de aplicações $g:(N, x) \rightarrow(\mathbb{R}, y)$ de classe $C^{\infty}$. Tomando uma parametrização de $N$ em $x$ podemos supor $N=\mathbb{R}^{n}$ e $x=0$. Definimos

$$
\varepsilon_{n}=\left\{f:\left(\mathbb{R}^{n}, 0\right) \rightarrow(\mathbb{R}, y) / f \text { é germe de aplicação } C^{\infty}\right\}
$$

Temos que $\varepsilon_{n}$ é um anel local cujo ideal maximal é

$$
m_{n}=\left\{f \in \varepsilon_{n} / f(0)=0\right\}
$$

Lema 1.3.1 (de Hadamard) Sejam U uma vizinhança convexa de $0 \mathrm{em}$ $\mathbb{R}^{n}$ e $f: U \times \mathbb{R}^{q} \rightarrow \mathbb{R}$ uma aplicação de classe $C^{\infty}$, tal que $f(0, y)=0$, $\forall y \in \mathbb{R}^{q}$. Então existem funções $f_{1}, \ldots, f_{n}$ definidas em $U \times \mathbb{R}^{q}$ tais que $f=x_{1} f_{1}+\ldots+x_{n} f_{n}$, onde $x_{1}, \ldots, x_{n}$ são as funções coordenadas de $\mathbb{R}^{n}$.

\section{Demonstração:}

Observemos que

$$
\int_{0}^{1} \frac{d}{d t} f\left(t x_{1}, \ldots, t x_{n}, y_{1}, \ldots, y_{q}\right) d t=f\left(x_{1}, \ldots, x_{n}, y_{1}, \ldots, y_{q}\right)-f\left(0, \ldots, 0, y_{1}, \ldots, y_{q}\right)
$$

Então

$$
\begin{aligned}
f(x, y) & =\int_{0}^{1} \frac{d}{d t} f\left(t x_{1}, \ldots, t x_{n}, y_{1}, \ldots, y_{q}\right) d t \\
& =\sum_{i=1}^{n} x_{i} \int_{0}^{1} \frac{\partial}{\partial x_{i}} f\left(t x_{1}, \ldots, t x_{n}, y_{1}, \ldots, y_{q}\right) d t
\end{aligned}
$$

Tomando $f_{i}=\int_{0}^{1} \frac{\partial}{\partial x_{i}} f\left(t x_{1}, \ldots, t x_{n}, y_{1}, \ldots, y_{n}\right) d t, i=1, \ldots, n$, temos $f=$ $\sum_{i=1}^{n} x_{i} f_{i}$

Usando o Lema de Hadamard (1.3.1) para $q=0$, mostramos $m_{n}=$ $\left\langle x_{1}, \ldots, x_{n}\right\rangle$, ou seja, o ideal gerado pelas funções coordenadas. 
Lema 1.3.2 (de Nakayama) Seja $R$ um anel comutativo com identidade 1. Seja $m$ um ideal de $R$ tal que $1+x$ é invertível em $R, \forall x \in m$. Sejam $M$ um $R$-módulo e $A$ e $B R$-submódulos com $A$ finitamente gerado. Se $A \subset B+m A$ então $A \subset B$.

\section{Demonstração:}

Sejam $a_{1}, \ldots, a_{t}$ geradores de $A$. Por hipótese, temos

$$
a_{i}=b_{i}+\lambda_{i 1} a_{1}+\ldots+\lambda_{i t} a_{t}
$$

onde $\lambda_{i j} \in m, i, j=1, \ldots, t$.

Seja $\Lambda=\left(\lambda_{i j}\right)$. Então

$$
(I-\Lambda)\left(\begin{array}{c}
a_{1} \\
\vdots \\
a_{t}
\end{array}\right)=\left(\begin{array}{c}
b_{1} \\
\vdots \\
b_{t}
\end{array}\right)
$$

Mas det $(I-\Lambda)=1-\lambda$, onde $\lambda \in m$. Logo det $(I-\Lambda)$ é invertível em $R$. Assim, $a_{i} \in B$. Portanto $A \subset B$.

\subsection{Grupo $\mathcal{R}$}

Seja $D_{n}=\left\{h:\left(\mathbb{R}^{n}, 0\right) \rightarrow\left(\mathbb{R}^{n}, 0\right) / h\right.$ é germe de difeomorfismo $\}$. O conjunto $D_{n}$ é um grupo com a operação de composição e age em $\varepsilon_{n}$ da seguinte maneira: $\phi: D_{n} \times \varepsilon_{n} \rightarrow \varepsilon_{n}$ e dada por $\phi(h, f)=f \circ h^{-1}$. Denotamos o grupo $D_{n}$ com tal ação por $\mathcal{R}$.

Definição 1.4.1 Sejam $f, g \in \varepsilon_{n}$. Dizemos que $f$ é $\mathcal{R}$-equivalente a $g$ se existe $h \in D_{n}$ tal que $g=f \circ h^{-1}$.

Definimos o ideal jacobiano de $f$ pelo ideal $J f=\left\langle\frac{\partial f}{\partial x_{1}}, \ldots, \frac{\partial f}{\partial x_{n}}\right\rangle$ de $\varepsilon_{n}$, gerado pelas derivadas parciais de $f$. O espaço tangente a $f$ segundo o grupo $\mathcal{R}$ é definido como o ideal $m_{n} J f$, e é denotado por $T \mathcal{R} f$.

Definições 1.4.2 Seja $f \in \varepsilon_{n}$. 
1. O espaço tangente estendido a $f$ segundo o grupo $\mathcal{R}$ é definido como o ideal $J f$, e é denotado por $T \mathcal{R}_{e} f$.

2. A $\mathcal{R}$-codimensão de $f$ é a dimensão de $\frac{m_{n}}{m_{n} J f}$ como espaço vetorial real e é denotada por $\mathcal{R}-\operatorname{cod} f$.

3. A $\mathcal{R}_{e}$-codimensão de $f$ é a dimensão de $\frac{\varepsilon_{n}}{J f}$ como espaço vetorial real e é denotada por $\mathcal{R}_{e}-\operatorname{cod} f$.

Definição 1.4.3 Definimos $D_{n}^{k}$ como o conjunto dos $k$-jatos de elementos de $D_{n} \subset J^{k}(n, n)$. Temos que $D_{n}^{k}$ é um grupo de Lie com a operação $*$ dada por

$$
j^{k} h_{1}(0) * j^{k} h_{2}(0)=j^{k}\left(h_{1} \circ h_{2}\right)(0)
$$

e age em $J^{k}(n, p)$ com a ação $\phi: D_{n}^{k} \times J^{k}(n, p) \rightarrow J^{k}(n, p)$ dada por $\phi(h, f)=$ $j^{k}\left(f \circ h^{-1}\right)(0)$. Denotamos o grupo $D_{n}^{k}$ com tal ação por $\mathcal{R}^{k}$.

Neste trabalho estamos interessados no caso em que $p=1$.

Definição 1.4.4 Sejam $f, g \in J^{k}(n, 1)$. Dizemos que $f$ e $g$ são $\mathcal{R}^{k}$-equivalentes, se existe $h \in D_{n}^{k}$ tal que $j^{k} f(0)=j^{k}\left(g \circ h^{-1}\right)(0)$. O espaço tangente à órbita de $f$ em $J^{k}(n, 1)$ é definido por

$$
T \mathcal{R}^{k} \cdot f=\frac{m_{n} J f}{m_{n}^{k+1}}
$$

Temos assim que $T \mathcal{R}^{k} f$ é gerado por $k$-jatos de elementos de $m_{n} J f$.

Proposição 1.4.5 Seja $f \in \varepsilon_{n}$. Então $\mathcal{R}-\operatorname{cod} f<\infty$ se, e somente se, existe um inteiro $k>0$ tal que $J f \supset m_{n}^{k}$, ou equivalentemente, $m_{n}^{k} \subset J f+$ $m_{n}^{k+1}$.

Proposição 1.4.6 Seja $f \in \varepsilon_{n}$ tal que $0<\mathcal{R}-\operatorname{cod} f<\infty$. Então

$$
\mathcal{R}-\operatorname{cod} f=\mathcal{R}_{e}-\operatorname{cod} f+n-1
$$

Definiremos $\varepsilon_{n, p}$ como o conjunto dos germes $f:\left(\mathbb{R}^{n}, 0\right) \rightarrow\left(\mathbb{R}^{p}, y\right)$. Observemos que $\varepsilon_{n, p}$ é um $\varepsilon_{n}$-módulo. 
Definição 1.4.7 Seja $f \in \varepsilon_{n, p}$ tal que $f(0)=0$. Definimos $f^{*}: \varepsilon_{p} \rightarrow \varepsilon_{n}$ por $f^{*}(g)=g \circ f$, e dizemos que $f^{*}$ é o homomorfismo induzido por $f$.

Observação 1.4.8 Seja $\varphi: \varepsilon_{n} \rightarrow \widehat{\varepsilon}_{n}$ dada por $f \mapsto \widehat{f}$, onde $\widehat{\varepsilon}_{n}$ é o conjunto das séries formais cujo termo constante é nulo que pode ser denotado por $\mathbb{R}\left[\left[x_{1}, \ldots, x_{n}\right]\right]$ e $\widehat{f}$ é a série de Taylor de $f$ sem o termo constante. Observemos que $\varphi$ é sobrejetora, mas não é injetora. Seja $I \subset \varepsilon_{n}$

1. A $\operatorname{dim} \frac{\varepsilon_{n}}{I}<\infty$ se, e somente se, $\operatorname{dim} \frac{\mathbb{R}\left[\left[x_{1}, \ldots, x_{n}\right]\right]}{\widehat{I}}<\infty$, onde $\widehat{I}=\varphi(I)$.

Além disso

2. $\operatorname{dim} \frac{\varepsilon_{n}}{I}=\operatorname{dim} \frac{\mathbb{R}\left[\left[x_{1}, \ldots, x_{n}\right]\right]}{\widehat{I}}$.

Proposição 1.4.9 Seja $f:\left(\mathbb{R}^{n}, 0\right) \rightarrow\left(\mathbb{R}^{n}, 0\right)$ um germe. Então $f^{*}: \varepsilon_{n} \rightarrow \varepsilon_{n}$ é um isomorfismo se, e somente se, $f$ é germe de difeomorfismo.

\subsection{Germes Finitamente Determinados}

Definição 1.5.1 Dizemos que $f \in \varepsilon_{n}$ é $k$-determinado se todo $g \in \varepsilon_{n}$ tal que $j^{k} g(0)=j^{k} f(0)$ é $\mathcal{R}$-equivalente a $f$. E que $f$ é finitamente determinado se $f$ é $k$-determinado para algum $k$.

Proposição 1.5.2 Se $f \in \varepsilon_{n}$ é tal que $m_{n}^{k} \subset m_{n} J f$, então $f$ é $k$-determinado.

Observação 1.5.3 Seja $f \in \varepsilon_{n}$.

1. Segue analogamente à Proposição 1.5.2 que se $m_{n}^{k+1} \subset m_{n}^{2} J f$, então $f$ é $k$-determinado

2. A hipótese $m_{n}^{k} \subset m_{n} J f$ da Proposição 1.5.2 pode ser substituída por $m_{n}^{k} \subset m_{n} J f+m_{n}^{k+1}$

3. A $\mathcal{R}$-codimensão de $f$ é finita se, e somente se, $f$ é finitamente determinado. 
Proposição 1.5.4 Seja $f \in m_{n}$. Se $f$ é $k$-determinado então $m_{n} J f=$ $T \mathcal{R} f \supset m_{n}^{k+1}$.

Proposição 1.5.5 Sejam $f, g \in \varepsilon_{n}$. Se $f$ e $g$ são $\mathcal{R}$-equivalentes e $f$ é $k$-determinado então $g$ é $k$-determinado.

Definição 1.5.6 Dizemos que um germe $f \in m_{n}$ tem uma singularidade não degenerada em 0 se

1. $d f_{0}=0$, isto é, $\frac{\partial f}{\partial x_{i}}(0)=0, i=1, \ldots, n$.

2. $\operatorname{det}\left(\frac{\partial^{2} f}{\partial x_{i} x_{j}}(0)\right) \neq 0$, ou seja, o rank da matriz $\left(\frac{\partial^{2}}{\partial x_{i} x_{j}}(0)\right)$ é $n$.

Neste caso dizemos que $f$ é Morse ou tem uma singularidade de Morse.

Proposição 1.5.7 Seja $f \in m_{n}$. Então $f$ tem uma singularidade não degenerada em 0 se, e somente se, $J f=m_{n}$.

Segue então o seguinte resultado:

Lema 1.5.8 (de Morse) Se $f \in m_{n}$ tem uma singularidade não degenerada em 0 então existe $h \in D_{n}$ tal que

$$
\left(f \circ h^{-1}\right)\left(x_{1}, \ldots, x_{n}\right)=-x_{1}^{2}-\ldots-x_{r}^{2}+x_{r+1}^{2}+\ldots+x_{n}^{2}
$$

\subsection{Desdobramentos e Deformações}

Definição 1.6.1 Seja $f_{0} \in m_{n} \varepsilon_{n, p}$. Um desdobramento a s-parâmetros de $f_{0}$ é um germe $F:\left(\mathbb{R}^{n} \times \mathbb{R}^{s},(0,0)\right) \rightarrow\left(\mathbb{R}^{p} \times \mathbb{R}^{s},(0,0)\right)$ dado por $(x, u) \mapsto$ $(f(x, u), u)$ tal que $f(x, 0)=f_{0}(x)$. O germe $f:\left(\mathbb{R}^{n} \times \mathbb{R}^{s},(0,0)\right) \rightarrow\left(\mathbb{R}^{p}, 0\right)$ é chamado deformação de $f_{0}$.

Vejamos o caso $p=1$ :

Definição 1.6.2 Duas deformações $f$ e $g$ a $s$-parâmetros de $f_{0}$ são isomorfas se existir um germe de difeomorfismo $\phi:\left(\mathbb{R}^{n} \times \mathbb{R}^{s}, 0\right) \rightarrow\left(\mathbb{R}^{n} \times \mathbb{R}^{s}, 0\right)$ tal que

1. $g=f \circ \phi$ 
2. $\phi(x, u)=(\psi(x, u), u)$ onde $\psi(x, 0)=x$

Definição 1.6.3 Uma deformação é dita trivial se é isomorfa à deformação constante dada por

$$
(x, u) \mapsto f_{0}(x)
$$

Seja $f$ uma deformação a $s$-parâmetros de $f_{0} \in m_{n}$. Seja $h:\left(\mathbb{R}^{q}, 0\right) \rightarrow\left(\mathbb{R}^{s}, 0\right)$ um germe de uma aplicação de classe $C^{\infty}$. Definimos $g:\left(\mathbb{R}^{n} \times \mathbb{R}^{q}, 0\right) \rightarrow(\mathbb{R}, 0)$ por $g(x, u)=f(x, h(u))$. Assim $g$ é uma deformação a $q$-parâmetros de $f_{0}$, que denotamos por $g=h^{*} f$. A deformação $h^{*} f$ é dita pull-back de $f$ por $h$.

Definição 1.6.4 Duas deformações $f$ e $g$ a $s$-parâmetros são ditas equivalentes se existir um germe de difeomorfismo $h:\left(\mathbb{R}^{s}, 0\right) \rightarrow\left(\mathbb{R}^{s}, 0\right)$ tal que $g$ é isomorfo a $h^{*} f$.

Definição 1.6.5 Se $\bar{g}$ é uma deformação a $q$-parâmetros, dizemos que $\bar{g}$ é induzida de $f$ se existir um germe $k:\left(\mathbb{R}^{q}, 0\right) \rightarrow\left(\mathbb{R}^{s}, 0\right)$ tal que $\bar{g}$ é isomorfa a $k^{*} f$.

Definição 1.6.6 Uma deformação $f$ de $f_{0} \in m_{n}$ é dita versal se qualquer outra deformação de $f_{0}$ é induzida de $f$.

Dizemos que $f$ é miniversal se for versal com o número mínimo de parâmetros.

Definição 1.6.7 Se $f:\left(\mathbb{R}^{n} \times \mathbb{R}^{s},(0,0)\right) \rightarrow(\mathbb{R}, 0)$ é uma deformação de $f_{0} \in m_{n}$ então as velocidades iniciais $\dot{f}_{i} \in \varepsilon_{n}, i=1, \ldots, s$, da deformação são definidas por $\dot{f}_{i}(x)=\frac{\partial f}{\partial u_{i}}(x, 0)$.

Proposição 1.6.8 $f:\left(\mathbb{R}^{n} \times \mathbb{R}^{s},(0,0)\right) \rightarrow(\mathbb{R}, 0)$ é uma deformação versal de $f_{0} \in m_{n}$ se, e somente se, a $\mathcal{R}$-codimensão de $f$ é finita $e$

$$
\varepsilon_{n}=J f_{0}+\mathbb{R}\left\{\dot{f}_{1}, \ldots, \dot{f}_{n}\right\}
$$




\section{Capítulo 2}

\section{Classificação de Germes de Funções}

O objetivo deste capítulo é classificar os germes simples de $\mathbb{R}^{n}$ em $\mathbb{R}$ através do Método da Transversal Completa.

Apresentamos o Método da Transversal Completa (seção 2.2) que também será usado nos capítulos seguintes.

As referências básicas são [1] e [9].

\subsection{Singularidades de corank $\leq 1$}

Temos do capítulo anterior que se $f \in m_{n}$, então $f$ tem codimensão zero se, e somente se, $f$ é não singular, isto é, submersão. Neste caso $f$ é $\mathcal{R}$-equivalente a

$$
\left(x_{1}, \ldots, x_{n}\right) \mapsto x_{1}
$$

Proposição 2.1.1 Seja $f \in m_{n}^{2}$, isto é, O é uma singularidade. Então $f$ tem $\mathcal{R}_{e}$-codimensão 1 se, e somente se, f tem uma singularidade não degenerada em 0 , ou seja, fé Morse. Neste caso $f$ é $\mathcal{R}$-equivalente a um germe da forma

$$
\left(x_{1}, \ldots, x_{n}\right) \mapsto-x_{1}^{2}-\ldots-x_{p}^{2}+x_{p+1}^{2}+\ldots+x_{n}^{2}
$$

\section{Demonstração:}




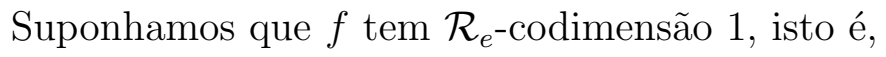

$$
\operatorname{dim} \frac{\varepsilon_{n}}{J f}=1=\operatorname{dim} \frac{\varepsilon_{n}}{m_{n}}
$$

Além disso, $J f \subset m_{n}$. Se $J f \subsetneq m_{n}$, temos

$$
\frac{\varepsilon_{n}}{J f} \supsetneq \frac{\varepsilon_{n}}{m_{n}}
$$

e então

$$
\operatorname{dim} \frac{\varepsilon_{n}}{J f}>\operatorname{dim} \frac{\varepsilon_{n}}{m_{n}}
$$

o que é uma contradição. Portanto $J f=m_{n}$. Segue da Proposição 1.5.7 que 0 é uma singularidade não degenerada. A recíproca é imediata. Como $f$ é 2-determinado, temos que $f$ é $\mathcal{R}$-equivalente a $j^{2} f(0)$, que é uma forma quadrática não degenerada. Segue de [7] que $f$ é $\mathcal{R}$-equivalente a

$$
\left(x_{1}, \ldots, x_{n}\right) \mapsto-x_{1}^{2}-\ldots-x_{p}^{2}+x_{p+1}^{2}+\ldots+x_{n}^{2}
$$

Definição 2.1.2 Seja $f \in m_{n}^{2}$ tal que $\mathcal{R}_{e}-\operatorname{cod} f \geqslant 2$. Então a matriz hessiana de $f$ é singular, logo tem rank $r<n$. O inteiro $c=n-r>0$ é chamado de corank de $f$ e denotado por corank $f$.

Proposição 2.1.3 (Lema da Decomposição ou Splitting Lemma) Seja $f \in m_{n}^{2}$ um germe finitamente determinado de corank c. Então $f$ é $\mathcal{R}$ equivalente a

$$
\left(x_{1}, \ldots, x_{n}\right) \mapsto h\left(x_{1}, \ldots, x_{c}\right)+\left( \pm x_{c+1}^{2} \pm \ldots \pm x_{n}^{2}\right)
$$

onde $h \in m_{c}^{3}$.

\section{Demonstração:}

Podemos encontrar coordenadas locais nas quais a submatriz

$$
\left(\frac{\partial^{2} f}{\partial x_{i} \partial x_{j}}(0)\right)_{c+1 \leq i, j \leq n}
$$

da matriz hessiana de $f$, é não singular. 
Assim a restrição de $f$ ao subespaço $\{0\} \times \mathbb{R}^{n-c}$ tem uma singularidade não degenerada na origem.

Seja $f_{0}=\left.f\right|_{\{0\} \times \mathbb{R}^{n-c}}$. Logo $f_{0} \in \varepsilon_{n-c}$ é Morse. Portanto $f_{0}$ é $\mathcal{R}$-equivalente a

$$
q\left(x_{c+1}, \ldots, x_{n}\right)=\sum_{i=c+1}^{n} \pm x_{i}^{2}
$$

Mas

$$
f\left(x_{1}, \ldots, x_{c}, x_{c+1}, \ldots, x_{n}\right)=f_{0}\left(x_{c+1}, \ldots, x_{n}\right)+f^{\prime}\left(x_{1}, \ldots, x_{n}\right)
$$

onde $f^{\prime}\left(0, \ldots, 0, x_{c+1}, \ldots, x_{n}\right)=0$.

Logo $f$ é $\mathcal{R}$-equivalente a

$$
g\left(x_{1}, \ldots, x_{n}\right)=q\left(x_{c+1}, \ldots, x_{n}\right)+\widetilde{f}\left(x_{1}, \ldots, x_{n}\right)
$$

em que $\widetilde{f}\left(0, \ldots, 0, x_{c+1}, \ldots, x_{n}\right)=0$. De fato, temos $q=f_{0} \circ \varphi$ onde $\varphi$ é germe de difeomorfismo nas variáveis $\left(x_{c+1}, \ldots, x_{n}\right)$. Seja $\psi\left(x_{1}, \ldots, x_{n}\right)=$ $\left(x_{1}, \ldots, x_{c}, \varphi\left(x_{c+1}, \ldots, x_{n}\right)\right)$. Assim

$$
\begin{aligned}
f \circ \psi\left(x_{1}, \ldots, x_{n}\right) & =f\left(x_{1}, \ldots, x_{c}, \varphi\left(x_{c+1}, \ldots, x_{n}\right)\right) \\
& =f_{0} \circ \varphi\left(x_{c+1}, \ldots, x_{n}\right)+f^{\prime} \circ \psi\left(x_{1}, \ldots, x_{n}\right) \\
& =q+\widetilde{f}\left(x_{1}, \ldots, x_{n}\right)
\end{aligned}
$$

onde $\tilde{f}=f^{\prime} \circ \psi$, e mais

$$
\begin{aligned}
\widetilde{f}\left(0, \ldots, 0, x_{c+1}, \ldots, x_{n}\right) & =f^{\prime} \circ \psi\left(0, \ldots, 0, x_{c+1}, \ldots, x_{n}\right) \\
& =f^{\prime}\left(0, \ldots, 0, \varphi\left(x_{c+1}, \ldots, x_{n}\right)\right)=0
\end{aligned}
$$

Temos que $g$ é uma deformação de $q$. Mas

$$
Q\left(u, x_{c+1}, \ldots, x_{n}\right)=q\left(x_{c+1}, \ldots, x_{n}\right)+u
$$

é uma deformação versal de $q$. Logo $g$ é induzida de $Q$, isto é, $g$ é equivalente (como deformação) a $h^{*} Q$ para algum $h:\left(\mathbb{R}^{c}, 0\right) \rightarrow(\mathbb{R}, 0)$. Em particular $g$ é equivalente (como germe) a

$$
\begin{gathered}
\left(h^{*} Q\right)\left(x_{1}, \ldots, x_{c}, x_{c+1}, \ldots, x_{n}\right)=Q\left(h\left(x_{1}, \ldots, x_{c}\right), x_{c+1}, \ldots, x_{n}\right)= \\
=q\left(x_{c+1}, \ldots, x_{n}\right)+h\left(x_{1}, \ldots, x_{c}\right)
\end{gathered}
$$

Como o corank de $f$ é $c$ segue que $h \in m_{c}^{3}$. 
Proposição 2.1.4 Os germes $f$ e $h$ do Splitting Lemma (2.1.3) têm a mesma $\mathcal{R}_{e}$-codimens ão.

\section{Demonstração:}

Temos que $\mathcal{R}_{e}-\operatorname{cod} f=\operatorname{dim} \frac{\varepsilon_{n}}{J f}=\operatorname{dim} \frac{\varepsilon_{n}}{\left\langle\frac{\partial h}{\partial x_{1}}, \ldots, \frac{\partial h}{\partial x_{c}}, x_{c+1}, \ldots, x_{n}\right\rangle}=\operatorname{dim} \frac{\varepsilon_{c}}{J h}=\mathcal{R}_{e}-\operatorname{cod} h$

Proposição 2.1.5 Seja $f \in m_{n}^{2}$ de corank 1 e $\mathcal{R}_{e}$-codimensão $k$-1. Então $f$ é $\mathcal{R}$-equivalente a

$$
\left(x_{1}, \ldots, x_{n}\right) \mapsto \pm x_{1}^{k} \pm x_{2}^{2} \pm \ldots \pm x_{n}^{2}
$$

Dizemos que $f$ é uma singularidade $A_{k-1}$.

\section{Demonstração:}

Segue do Splitting Lemma (2.1.3) que $f$ é $\mathcal{R}$-equivalente a

$$
\left(x_{1}, \ldots, x_{n}\right) \mapsto h\left(x_{1}\right) \pm \ldots \pm x_{n}^{2}
$$

onde $h \in m_{1}^{3}$ e $\mathcal{R}_{e}-\operatorname{cod} h=k-1$.

Suponhamos que $h \in m_{1}^{j}, \forall j$. Logo $\frac{\varepsilon_{1}}{J h} \supset \frac{\varepsilon_{1}}{m_{1}^{j}}, \forall j$. Portanto $\operatorname{dim} \frac{\varepsilon_{1}}{J h} \geq j$,

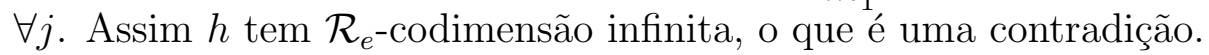

Então existe um inteiro $j$ tal que $h \in m_{1}^{j}$ e $h \notin m_{1}^{j+1}$. Logo segue do Lema de Hadamard que

$$
h\left(x_{1}\right)=x_{1}^{j} g\left(x_{1}\right)
$$

onde $g(0) \neq 0$. Temos

$$
k-1=\mathcal{R}_{e}-\operatorname{cod} h=\operatorname{dim} \frac{\varepsilon_{1}}{\left\langle j x^{j-1} g+x_{1}^{j} \frac{d g}{d x_{1}}\right\rangle}=\operatorname{dim} \frac{\varepsilon_{1}}{\left\langle x^{j-1}\left(j g+x_{1} \frac{d g}{d x_{1}}\right)\right\rangle}
$$

Como $\left(j g+x_{1} \frac{d g}{d x_{1}}\right)(0) \neq 0$, o germe $j g+x_{1} \frac{d g}{d x_{1}}$ é invertível, logo

$$
k-1=\operatorname{dim} \frac{\varepsilon_{1}}{\left\langle x^{j-1}\left(j g+x_{1} \frac{d g}{d x_{1}}\right)\right\rangle}=\operatorname{dim} \frac{\varepsilon_{1}}{\left\langle x^{j-1}\right\rangle}=j-1
$$


Obtemos assim que $j=k$ e $h\left(x_{1}\right)=x_{1}^{k} g\left(x_{1}\right) \operatorname{com} g(0) \neq 0$. Então

$$
\frac{\partial h}{\partial x_{1}}=x_{1}^{k-1}\left[k g+x_{1} \frac{\partial g}{\partial x_{1}}\right]
$$

Seja $\bar{h}=k g+x_{1} \frac{\partial g}{\partial x_{1}}$, temos que $\bar{h}$ não se anula em $x_{1}=0, \log$ o $\bar{h}$ é invertível. Obtemos assim $J h=\left\langle x_{1}^{k-1}\right\rangle=m_{1}^{k-1}$, conseqüentemente $m_{1} J h=$ $m_{1}^{k}$. Pela Proposição 1.5.2 temos que $h$ é $k$-determinado, além disso $j^{k} h(0)=$ $c x_{1}^{k}$ onde $c=g(0)$. Portanto $h$ é $\mathcal{R}$-equivalente a $x_{1}^{k}$.

\subsection{O Método da Transversal Completa}

Proposição 2.2.1 (Transversal Completa) Sejam G um grupo de Lie agindo num espaço vetorial $V$ e $H \subset V$ um subespaço tal que

$$
g \cdot(\alpha+\beta)=g \cdot \alpha+\beta
$$

$\forall \alpha \in V, \forall \beta \in H \quad e \forall g \in G$. Então

i) $\forall \alpha \in V$ temos

$$
G \cdot \alpha \cap(\alpha+H) \supset \alpha+\left(T_{\alpha} G \cdot \alpha \cap H\right)
$$

ii) se $T \subset H$ é um subespaço tal que

$$
H \subset T+T_{\alpha} G \cdot \alpha
$$

então $\forall \beta \in H, \alpha+\beta$ está na mesma órbita que $\alpha+\beta^{\prime}$ para algum $\beta^{\prime} \in T$.

\section{Demonstração:}

i) Seja $N=\alpha+\left(T_{\alpha} G \cdot \alpha \cap H\right)$. Temos que $N \subset \alpha+H$. Resta mostrar que $N$ está contido numa única órbita. Para isto usaremos o Lema de Mather (1.2.6).

Afirmamos que $T_{\alpha+\beta} G \cdot(\alpha+\beta)=T_{\alpha} G \cdot \alpha, \forall \beta \in H$ e $\forall \alpha \in V$. De fato, temos que $T_{\alpha+\beta} G \cdot(\alpha+\beta)=d \phi_{\alpha+\beta}(1)\left(T_{1} G\right)$ e $T_{\alpha} G \cdot \alpha=d \phi_{\alpha}(1)\left(T_{1} G\right)$ onde $\phi: G \times V \rightarrow V$ é a ação, $\phi(g, \alpha)=g \cdot \alpha, \phi_{\alpha}: G \rightarrow V$ é dada por $\phi_{\alpha}(g)=g \cdot \alpha$, $\forall \alpha \in V$. 
Seja $\lambda:(-\varepsilon, \varepsilon) \rightarrow G$ tal que $\lambda(0)=1$. Então $\lambda^{\prime}(0) \in T_{1} G$. Temos

$$
\begin{aligned}
\left(\phi_{\alpha+\beta} \circ \lambda\right)^{\prime}(0) & =\lim _{t \rightarrow 0} \frac{\left(\phi_{\alpha+\beta} \circ \lambda\right)(t)-\left(\phi_{\alpha+\beta} \circ \lambda\right)(0)}{t} \\
& =\lim _{t \rightarrow 0} \frac{\lambda(t) \cdot(\alpha+\beta)-(\alpha+\beta)}{t} \\
& =\lim _{t \rightarrow 0} \frac{\lambda(t) \cdot \alpha+\beta-\alpha-\beta}{t} \\
& =\lim _{t \rightarrow 0} \frac{\left(\phi_{\alpha} \circ \lambda\right)(t)-\left(\phi_{\alpha} \circ \lambda\right)(0)}{t} \\
& =\left(\phi_{\alpha} \circ \lambda\right)^{\prime}(0)
\end{aligned}
$$

Portanto $T_{\alpha+\beta} G \cdot(\alpha+\beta)=T_{\alpha} G \cdot \alpha, \forall \beta \in H, \forall \alpha \in V$.

Seja $y \in N$. Então $y=\alpha+\beta$, onde $\beta \in T_{\alpha} G \cdot \alpha \cap H$. Temos

$$
T_{y} G \cdot y=T_{\alpha+\beta} G \cdot(\alpha+\beta)=T_{\alpha} G \cdot \alpha \supset T_{\alpha} G \cdot \alpha \cap H=T_{y} N
$$

Ainda, $\operatorname{dim} T_{y} G \cdot y=\operatorname{dim} T_{\alpha} G \cdot \alpha, \log$ o é constante $\forall y \in N$. Segue do Lema de Mather (1.2.6) que $\mathrm{N}$ está contido numa única órbita, isto é, $N \subset G \cdot \alpha$.

ii) Temos pelo item i) que

$$
G \cdot(\alpha+\beta) \cap(\alpha+\beta+H) \supset \alpha+\beta+\left(T_{\alpha+\beta} G \cdot(\alpha+\beta) \cap H\right)=\alpha+\beta+\left(T_{\alpha} G \cdot \alpha \cap H\right)
$$

para todo $\alpha \in V, \beta \in H$.

Assim,

$$
\begin{aligned}
\cup_{\beta \in T}(G \cdot(\alpha+\beta)) & \supset \cup_{\beta \in T}\left(\alpha+\beta+\left(T_{\alpha} G \cdot \alpha \cap H\right)\right) \\
& =\alpha+T+\left(T_{\alpha} G \cdot \alpha \cap H\right) \\
& \supset \alpha+H
\end{aligned}
$$

Seja $\bar{\beta} \in H . \quad \operatorname{Logo} \alpha+\bar{\beta} \in \alpha+H \subset \cup_{\beta \in T} G \cdot(\alpha+\beta)$. Então $\alpha+\bar{\beta} \in$ $G \cdot\left(\alpha+\beta^{\prime}\right)$ para algum $\beta^{\prime} \in T$.

Definição 2.2.2 O subespaço $T$ da Proposição 2.2.1 é chamado transversal completa. 
Exemplo 2.2.3 Sejam $G=\mathcal{R}^{k}$, o conjunto dos $k$-jatos de elementos de $\mathcal{R}$, e $V=J^{k}(n, 1)$. Consideremos a ação de $\mathcal{R}^{k}$ em $V$. Seja $H^{k}(n, 1)$ o subespaço dos polinômios homogêneos de grau $k$ em $n$ variáveis. Tomando $h \in H^{k}(n, 1)$, $f \in J^{k}(n, 1)$ e $\varphi \in \mathcal{R}^{k}$, temos

$\varphi \cdot(f+h)=j^{k}((f+h) \circ \varphi)(0)=j^{k}(f \circ \varphi+h \circ \varphi)(0)=\varphi \cdot f+h \circ j^{1} \varphi(0)=\varphi \cdot f+h$

se $j^{1} \varphi(0)$ for a identidade.

Denotemos por $\mathcal{R}_{1}$ o subgrupo de $\mathcal{R}$ constituído dos germes de difeomorfismos cujo 1-jato é a identidade e $\mathcal{R}_{1}^{k}$ é o conjunto dos $k$-jatos de elementos de $\mathcal{R}_{1}$. Logo a ação de $\mathcal{R}_{1}^{k}$ em $J^{k}(n, 1)$ satisfaz as hipóteses da Proposição 2.2.1.

Observação 2.2.4 Se $f \in m_{n}$, temos que $T \mathcal{R}_{1} f=m_{n}^{2} J f$. Logo

$$
T \mathcal{R}_{1}^{k} j^{k} f(0)=\frac{m_{n}^{2} J f+m_{n}^{k+1}}{m_{n}^{k+1}}
$$

Proposição 2.2.5 (Transversal Completa para jatos) Sejam $f \in J^{k}(n, 1)$, $H^{k+1}(n, 1) \subset J^{k+1}(n, 1)$, o subespaço dos polinômios homogêneos de grau $k+1$ em $n$ variáveis, e $T \subset H^{k+1}(n, 1)$ subespaço tais que

$$
m_{n}^{2} J f+T+m_{n}^{k+2} \supset m_{n}^{k+1}
$$

Então qualquer $g \in J^{k+1}(n, 1)$ com $j^{k} g(0)=f$ é $\mathcal{R}_{1}^{k}$-equivalente a $f+\beta$ com $\beta \in T$. Se $T=\mathbb{R}\left\{G_{1}, \ldots, G_{r}\right\}, \operatorname{com} G_{i} \in H^{k+1}(n, 1), i=1, \ldots, r$, isto é, o subespaço vetorial gerado por $G_{1}, \ldots, G_{r}$, então $g$ é $\mathcal{R}_{1}^{k}$-equivalente a $f+\sum_{i=1}^{r} a_{i} G_{i}$. Se $r=0$ e $N \geq k+1$ então qualquer $N$-jato g com $j^{k} g(0)=f$ é $\mathcal{R}_{1}^{N}$-equivalente a $f$.

\section{Demonstração:}

Mostremos que $H^{k+1}(n, 1) \subset T+T \mathcal{R}_{1}^{k+1} f$. Pela hipótese temos que

$$
H^{k+1}(n, 1)=\frac{m_{n}^{k+1}}{m_{n}^{k+2}} \subset \frac{m_{n}^{2} J f+m_{n}^{k+2}+T}{m_{n}^{k+2}}=T \mathcal{R}_{1}^{k+1} f+T
$$

Conseqüentemente, se $g \in J^{k+1}(n, 1)$ com $j^{k} g(0)=f$, temos que $g=$ $f+\beta$, onde $\beta \in H^{k+1}(n, 1)$. 
Usando a Proposição 2.2.1 temos que $g=f+\beta$ é $\mathcal{R}_{1}^{k}$-equivalente a $f+\beta^{\prime}$ para algum $\beta^{\prime} \in T$.

Agora no caso $r=0$ e $N \geq k+1$, mostremos que $m_{n}^{2} J f+m_{n}^{N+1} \supset m_{n}^{N}$.

Seja $x_{1}^{i_{1}} \ldots x_{n}^{i_{n}} \in m_{n}^{N}, i_{1}+\cdots+i_{n}=N$. Temos que $x_{1}^{i_{1}} \ldots x_{n}^{i_{n}}=\alpha \beta$ com $\alpha \in m_{n}^{N-k-1}$ e $\beta \in m_{n}^{k+1}$.

Por hipótese, temos $\alpha \beta=\alpha(h+\gamma)$, com $h \in m_{n}^{2} J f$ e $\gamma \in m_{n}^{k+2}$. Assim $\alpha(h+\gamma)=\alpha h+\alpha \gamma \in m_{n}^{2} J f+m_{n}^{N+1}$. Portanto $H^{N}(n, 1) \subset T \mathcal{R}_{1}^{N} f$.

Se $g \in J^{N}(n, 1)$ e $j^{k} g(0)=f$, temos que $g=f+\beta$, com $\beta \in H^{N}(n, 1)$. Concluímos assim, pelos resultados acima, que $g$ é $\mathcal{R}_{1}^{N}$-equivalente a $f$.

\subsection{Singularidades de Corank 2}

Começaremos esta seção apresentando a seguinte:

Proposição 2.3.1 Seja $p \in H^{3}(2,1)$. Então existe um isomorfismo linear $\tau: \mathbb{R}^{2} \rightarrow \mathbb{R}^{2}$ tal que $p \circ \tau$ tem uma das seguintes formas:

$$
0, x^{3}, x y^{2}, x^{3} \pm x y^{2}
$$

e estas formas são as únicas, isto é, duas formas em (2.1) não são linearmente equivalentes.

\section{Demonstração:}

Mostremos primeiramente a unicidade: duas formas em (2.1) não são linearmente equivalentes, isto é, não podem ser transformadas uma na outra através de um isomorfismo linear $\tau: \mathbb{R}^{2} \rightarrow \mathbb{R}^{2}$.

Temos que a forma nula é invariante sob $\tau$, assim só as outras formas têm que ser consideradas.

Consideremos o conjunto dos zeros e o conjunto dos pontos críticos destas formas:

\begin{tabular}{|c|c|c|}
\hline & Conjunto de zeros & Conjunto de pontos críticos \\
\hline$x^{3}$ & $\{x=0\}$ & $\{x=0\}$ \\
$x y^{2}$ & $\{x=0\} \cup\{y=0\}$ & $\{x=0\}$ \\
$x^{3}-x y^{2}$ & $\{x=0\} \cup\{x=y\} \cup\{x=-y\}$ & $\{0\}$ \\
$x^{3}+x y^{2}$ & $\{x=0\}$ & $\{0\}$ \\
\hline
\end{tabular}


Afirmamos que se $p, q \in H^{3}(2,1)$ são tais que $p \circ \tau=q$ então $\tau$ leva o conjunto de zeros de $q$ no conjunto de zeros de $p$. De fato, seja $(x, y) \in \mathbb{R}^{2}$ tal que $q(x, y)=0$. Então $p \circ \tau(x, y)=0$, assim $\tau(x, y) \in p^{-1}(0)$, isto é $\tau\left(q^{-1}(0)\right) \subset p^{-1}(0)$. Como $q \circ \tau^{-1}=p$, podemos raciocinar analogamente para mostrar que $p^{-1}(0) \subset \tau\left(q^{-1}(0)\right)$.

Além disso, $\tau$ leva o conjunto dos pontos críticos de $q$ no conjunto dos pontos críticos de $p$. De fato, temos que $\tau(x, y)=(\alpha x+\beta y, \gamma x+\delta y)$ com $\alpha \delta-\beta \gamma \neq 0$. Assim

$$
\begin{aligned}
& \frac{\partial q}{\partial x}\left(x_{0}, y_{0}\right)=\frac{\partial p \circ \tau}{\partial x}\left(x_{0}, y_{0}\right)=\frac{\partial p}{\partial x}\left(\tau\left(x_{0}, y_{0}\right)\right) \alpha+\frac{\partial p}{\partial y}\left(\tau\left(x_{0}, y_{0}\right)\right) \gamma \\
& \frac{\partial q}{\partial y}\left(x_{0}, y_{0}\right)=\frac{\partial p \circ \tau}{\partial y}\left(x_{0}, y_{0}\right)=\frac{\partial p}{\partial x}\left(\tau\left(x_{0}, y_{0}\right)\right) \beta+\frac{\partial p}{\partial y}\left(\tau\left(x_{0}, y_{0}\right)\right) \delta
\end{aligned}
$$

Colocando na forma matricial temos

$$
\left(\begin{array}{l}
\frac{\partial q}{\partial x}\left(x_{0}, y_{0}\right) \\
\frac{\partial q}{\partial y}\left(x_{0}, y_{0}\right)
\end{array}\right)=\left(\begin{array}{ll}
\alpha & \gamma \\
\beta & \delta
\end{array}\right)\left(\begin{array}{l}
\frac{\partial p}{\partial x}\left(\tau\left(x_{0}, y_{0}\right)\right) \\
\frac{\partial p}{\partial y}\left(\tau\left(x_{0}, y_{0}\right)\right)
\end{array}\right)
$$

Portanto, se $\left(x_{0}, y_{0}\right)$ é ponto crítico de $q$ temos que $\tau\left(x_{0}, y_{0}\right)$ é ponto crítico de $p$, e reciprocamente, se $\left(x_{1}, y_{1}\right)$ é ponto crítico de $p$, temos que existe $\left(x_{0}, y_{0}\right) \in \mathbb{R}^{2}$ tal que $\left(x_{1}, y_{1}\right)=\tau\left(x_{0}, y_{0}\right)$. Assim $\left(x_{0}, y_{0}\right)$ é ponto crítico de $q$.

Observando a tabela acima temos que duas formas em (2.1) ou seus conjuntos de zeros ou seus conjuntos de pontos críticos não podem ser transformados um no outro através de um isomorfismo linear. Com isso concluímos que quaisquer duas formas em (2.1) são não linearmente equivalentes.

Mostremos agora que todo $p \in H^{3}(2,1)$ é linearmente equivalente a uma das formas em (2.1). Sejam $p(x, y)=a x^{3}+b x^{2} y+c x y^{2}+d y^{3}, \tau(x, y)=$ $(\alpha x+\beta y, \delta x+\gamma y)$, com $\alpha \delta-\beta \gamma \neq 0$ e $p^{\prime}(x, y)=p(\tau(x, y))=a^{\prime} x^{3}+b^{\prime} x^{2} y+$ $c^{\prime} x y^{2}+d^{\prime} y^{3}, \mathrm{com}$

$$
\begin{aligned}
& a^{\prime}=a \alpha^{3}+b \alpha^{2} \gamma+c \alpha \gamma^{2}+d \gamma^{3} \\
& b^{\prime}=3 a \alpha^{2} \beta+b\left(2 \alpha \beta \gamma+\alpha^{2} \delta\right)+c\left(2 \alpha \gamma \delta+\beta \gamma^{2}\right)+3 d \gamma^{2} \delta
\end{aligned}
$$




$$
\begin{aligned}
& c^{\prime}=3 a \alpha \beta^{2}+b\left(2 \alpha \beta \delta+\beta^{2} \gamma\right)+c\left(2 \beta \gamma \delta+\alpha \delta^{2}\right)+3 d \gamma \delta^{2}, \\
& d^{\prime}=a \beta^{3}+b \beta^{2} \delta+c \beta \delta^{2}+d \delta^{3} .
\end{aligned}
$$

Podemos escolher $\tau$ tal que $d^{\prime}=0$, pois se $a=0$, tomamos $\alpha=\delta=$ 0 e $\beta=\gamma=1$; se $a \neq 0$, tomamos $\alpha=\delta=1, \gamma=0$ e $\beta$ é tal que $a \beta^{3}+b \beta^{2}+c \beta+d=0$. Conseqüentemente, podemos tomar $p$ da seguinte forma

$$
p(x, y)=a x^{3}+b x^{2} y+c x y^{2}
$$

Agora $\tau$ pode ser escolhido de forma que $b^{\prime}=d^{\prime}=0$, pois se $c \neq 0$, tomamos $\alpha=\delta=1, \beta=0$ e $\gamma=-\frac{b}{2 c}$; e quando $c=0$ temos dois casos: se $b \neq 0$, podemos tomar $\alpha=0, \beta=\gamma=1, \delta=-\frac{a}{b}$ e se $b=0$ podemos tomar $\alpha=1, \beta=0, \delta=1$ e $\gamma=1$.

Assim não há nenhuma restrição em assumir $p$ seja da forma

$$
p(x, y)=a x^{3}+c x y^{2}
$$

Observemos agora os seguintes casos:

- se $a=0$ e $c=0$, então $p(x, y)=0$;

- se $a=0$ e $c \neq 0$, então $p(x, y)=c x y^{2}$, se $\tau(x, y)=\left(\frac{x}{c}, y\right)$, temos $p \circ \tau(x, y)=x y^{2}$

- se $a \neq 0$ e $c=0$, então $p(x, y)=a x^{3}$, se $\tau(x, y)=\left(\left(\frac{1}{a}\right)^{1 / 3} x, y\right)$, temos $p \circ \tau(x, y)=x^{3}$

- se $a \neq 0$ e $c \neq 0$, temos $p(x, y)=a x^{3}+c x y^{2}$, assim se $\frac{c}{a}>0$, tomando $\tau(x, y)=\left(\left(\frac{1}{a}\right)^{1 / 3} x,\left(\frac{a^{1 / 3}}{c}\right)^{1 / 2} y\right)$, obtemos $p \circ \tau(x, y)=x^{3}+x y^{2}$, e se $\frac{c}{a}<0$, tomando $\tau(x, y)=\left(\left(\frac{1}{a}\right)^{1 / 3} x,\left(-\frac{a^{1 / 3}}{c}\right)^{1 / 2} y\right)$, obtemos $p \circ \tau(x, y)=$ $x^{3}-x y^{2}$.

Seja $f \in m_{n}^{2}$ um germe finitamente determinado de corank 2. Segue do Splitting Lemma (2.1.3) que $f$ é $\mathcal{R}$-equivalente a

$$
\left(x, y, x_{3}, \ldots, x_{n}\right) \mapsto g(x, y) \pm x_{3}^{2} \pm \ldots \pm x_{n}^{2}
$$


onde $g \in m_{2}^{3}$, isto é, $j^{2} g(0)=0$. Assim classificar $f$ é equivalente a classificar g. Suponhamos $j^{3} g(0)=a x^{3}+b x^{2} y+c x y^{2}+d y^{3}$. Consideremos $J^{3}(2,1)$ com a ação de $\mathcal{R}^{3}$. Segue da Proposição 2.3.1 que em $H^{3}(2,1)$ temos as seguintes $\mathcal{R}^{3}$-órbitas: $x^{3} \pm x y^{2}, x y^{2}, x^{3}, 0$. Consideremos os seguintes casos:

- $j^{3} g(0)=x^{3} \pm x y^{2}$. Seja $h(x, y)=x^{3} \pm x y^{2}$. Temos que

$$
m_{2} J h=m_{2}\left\langle 3 x^{2} \pm y^{2}, x y\right\rangle=\left\langle x^{3}, x^{2} y, x y^{2}, y^{3}\right\rangle=m_{2}^{3}
$$

Segue da Proposição 1.5.2 que $h$ é 3-determinado. Portanto $g$ é $\mathcal{R}$ equivalente a $h$ e $f$ é $\mathcal{R}$-equivalente a $\left(x, y, x_{3}, \ldots, x_{n}\right) \mapsto x^{3} \pm x y^{2} \pm$ $x_{3}^{2} \pm \ldots \pm x_{n}^{2}$. Esta singularidade é chamada $D_{4}$. Ainda

$$
\begin{aligned}
\mathcal{R}_{e}-\operatorname{cod} f & =\operatorname{dim} \frac{\varepsilon_{2}}{\left\langle 3 x^{2} \pm y^{2}, x y\right\rangle} \\
& =\operatorname{dim} \frac{\varepsilon_{2}}{\left\langle 3 x^{2} \pm y^{2}, x\right\rangle}+\operatorname{dim} \frac{\varepsilon_{2}}{\left\langle 3 x^{2} \pm y^{2}, y\right\rangle} \\
& =4
\end{aligned}
$$

- $j^{3} g(0)=x y^{2}$. Seja $h(x, y)=x y^{2}$. Observemos que, como

$$
\operatorname{dim} \frac{\mathbb{R}[[x, y]]}{\left\langle y^{2}, x y\right\rangle}=\operatorname{dim} \mathbb{R}\left\{1, x, y, x^{2}, x^{3}, \ldots\right\}=\infty
$$

então $\mathcal{R}_{e}-\operatorname{cod} h=\operatorname{dim} \frac{\varepsilon_{2}}{\left\langle y^{2}, x y\right\rangle}=\infty$.

Queremos determinar as órbitas em $J^{4}(2,1)$, segundo $\mathcal{R}^{4}$, de elementos cujo 3 -jato é $x y^{2}$. Para isto, vamos calcular a transversal completa $T$ :

$$
m_{2}^{2} J h+T+m_{2}^{5} \supset m_{2}^{4}
$$

Temos:

$$
m_{2}^{2} J h=m_{2}^{2}\left\langle y^{2}, x y\right\rangle=\left\langle x^{2} y^{2}, x^{3} y, x y^{3}, y^{4}\right\rangle \subset m_{2}^{4}
$$

Então $T=\mathbb{R}\left\{x^{4}\right\}$. Pela Proposição 2.2.5, temos que $j^{4} g(0)$ é $\mathcal{R}^{4}$ equivalente a $x y^{2}+a x^{4}$. Seja $\varphi(x, y)=x y^{2}+a x^{4}$. 
Se $a>0, \varphi\left(\frac{x}{a^{\frac{1}{4}}}, a^{\frac{1}{8}} y\right)=x y^{2}+x^{4}$. E se $a<0, \varphi\left(\frac{x}{(-a)^{\frac{1}{4}}},(-a)^{\frac{1}{8}} y\right)=$ $x y^{2}-x^{4}$. Logo $j^{4} g(0)$ é $\mathcal{R}^{4}$-equivalente a $x y^{2}+x^{4}, x y^{2}-x^{4}$ ou $x y^{2}$.

Temos que $\bar{\varphi}(x, y)=x y^{2} \pm x^{4}$ é 4-determinado, pois

$$
m_{2} J \bar{\varphi}=m_{2}\left\langle y^{2} \pm 4 x^{3}, x y\right\rangle=\left\langle x^{4}, x y^{2}, x^{2} y, y^{3}\right\rangle \supset m_{2}^{4}
$$

Logo $g$ é $\mathcal{R}$-equivalente a $x y^{2} \pm x^{4}$. Esta singularidade é chamada $D_{5}$. Além disso

$$
\mathcal{R}_{e}-\operatorname{cod} \bar{\varphi}=\operatorname{dim} \frac{\varepsilon_{2}}{J \bar{\varphi}}=\operatorname{dim} \frac{\varepsilon_{2}}{\left\langle y^{2} \pm 4 x^{3}, x y\right\rangle}=5
$$

Prosseguindo desta maneira, suponhamos que $j^{k} g(0)=x y^{2}, k \geq 5$. Queremos determinar as órbitas em $J^{k+1}(2,1)$ de elementos cujo $k$-jato é $x y^{2}$. Para isto calculamos primeiro a transversal completa $T$ :

$$
m_{2}^{2}\left\langle y^{2}, x y\right\rangle+T+m_{2}^{k+2} \supset m_{2}^{k+1}
$$

que é $T=\mathbb{R}\left\{x^{k+1}\right\}$. Segue da Proposição 2.2 .5 que $j^{k+1} g(0)$ é $\mathcal{R}^{k+1}$ _ equivalente a $x y^{2}+\alpha x^{k+1}$. Após uma mudança linear de coordenadas obtemos as seguintes $\mathcal{R}^{k+1}$-órbitas: $x y^{2} \pm x^{k+1}$ e $x y^{2}$.

Temos que $x y^{2} \pm x^{k+1}$ é $k+1$-determinado, $\log g$ é $\mathcal{R}$-equivalente a $x y^{2} \pm x^{k+1}$, e esta singularidade é chamada $D_{k+2}$. Além disso, tem $\mathcal{R}_{e}$-codimensão $k+2$.

- $j^{3} g(0)=x^{3}$. Temos que $\mathcal{R}_{e}-\operatorname{cod} j^{3} g(0)=\infty$. Calculemos então a transversal completa $T$

$$
m_{2}^{2}\left\langle x^{2}\right\rangle+T+m_{2}^{5} \supset m_{2}^{4}
$$

$\operatorname{assim} T=\mathbb{R}\left\{x y^{3}, y^{4}\right\}$. Segue da Proposição 2.2 .5 que todo polinômio em $J^{4}(2,1)$ cujo 3 -jato é $x^{3}$ é $\mathcal{R}^{4}$-equivalente a $x^{3}+a x y^{3}+b y^{4}$.

Aqui temos três casos:

1. $b \neq 0$. Então $x^{3}+a x y^{3}+b y^{4}$ é $\mathcal{R}^{4}$-equivalente a $x^{3}+\bar{a} x y^{3} \pm y^{4}$. Vamos aplicar o Lema de Mather (1.2.6) para $M=J^{4}(2,1), G=$ $\mathcal{R}^{4}$ e $N=\left\{p \in J^{4}(2,1) / p=x^{3}+a x y^{3}+y^{4}, a \in \mathbb{R}\right\}$. 
Temos que $N$ é uma variedade conexa e se $p \in N$, então $T_{p} N=$ $\mathbb{R}\left\{x y^{3}\right\}$. Observemos que

$$
T_{p} G \cdot p=\frac{m_{2}\left\langle 3 x^{2}+a y^{3}, 3 a x y^{2}+4 y^{3}\right\rangle+m_{2}^{5}}{m_{2}^{5}}
$$

Como $x\left(4 y^{3}+3 a x y^{2}\right)-a y^{2}\left(3 x^{2}+a y^{3}\right)=4 x y^{3}-a^{2} y^{5} \in m_{2} J p$ então $x y^{3} \in T_{p} G \cdot p$.

Além disso $\operatorname{dim} T_{p} G \cdot p=\operatorname{dim} \mathbb{R}\left\{x^{4}, x^{3} y, x^{2} y^{2}, x y^{3}, y^{4}, x^{3}, x^{2} y\right\}=7$.

Segue do Lema de Mather que $N$ está contida numa única órbita. Portanto $x^{3}+a x y^{3} \pm y^{4}$ é $\mathcal{R}^{4}$-equivalente a $x^{3} \pm y^{4}$.

Temos que $x^{3} \pm y^{4}$ é 4 -determinado, assim $g$ é $\mathcal{R}$-equivalente a $x^{3} \pm$ $y^{4}$. A singularidade $x^{3} \pm y^{4}$ é chamada $E_{6}$ e tem $\mathcal{R}_{e}$-codimensão 6 .

2. $b=0$ e $a \neq 0$. Temos $j^{4} g(0)$ é $\mathcal{R}^{4}$-equivalente a $x^{3}+x y^{3}$. Seja $h(x, y)=x^{3}+x y^{3}$. Observemos que

$$
m_{2} J h=\left\langle x^{3}, x^{2} y^{2}, x y^{3}, 3 x^{2} y+y^{4}\right\rangle \supset m_{2}^{5}
$$

Portanto, $h$ é 5 -determinado.

Consideremos em $J^{5}(2,1)$ os polinômios cujo 4 -jato é $x^{3}+x y^{3}$. Calculemos então a transversal completa $T$ :

$$
m_{2}^{2}\left\langle 3 x^{2}+y^{3}, 3 x y^{2}\right\rangle+T+m_{2}^{6} \supset m_{2}^{5}
$$

Obtemos assim que $T=\mathbb{R}\left\{y^{5}\right\}$. Se $p \in J^{5}(2,1)$ é tal que $j^{4} p(0)=$ $x^{3}+x y^{3}$, segue da Proposição 2.2.5 que $p$ é $\mathcal{R}^{5}$-equivalente a $x^{3}+$ $x y^{3}+a y^{5}$.

Vamos aplicar o Lema de Mather para $M=J^{5}(2,1), G=\mathcal{R}^{5}$ e $N=\left\{p \in J^{5}(2,1) / p=x^{3}+x y^{3}+a y^{5}, a \in \mathbb{R}\right\}$. Temos que $N$ é subvariedade conexa e $T_{p} N=\mathbb{R}\left\{y^{5}\right\}, \forall p \in N$. Ainda,

$$
T_{p} G \cdot p=\frac{m_{2}\left\langle 3 x^{2}+y^{3}, 3 x y^{2}+5 a y^{4}\right\rangle+m_{2}^{6}}{m_{2}^{6}}
$$

Além disso,

$$
\begin{gathered}
\operatorname{dim} T_{p} G \cdot p=\operatorname{dim} \mathbb{R}\left\{x^{3}, x^{4}, x^{3} y, x^{2} y^{2}, x y^{3}, x^{5}, x^{4} y, x^{3} y^{2},\right. \\
\left.x^{2} y^{3}, x y^{4}, y^{5}, 3 x^{2} y+y^{4}\right\}=12
\end{gathered}
$$


para todo $p \in N$. Segue do Lema de Mather que N está contida numa única órbita. Daí $x^{3}+x y^{3}+a y^{5}$ é $\mathcal{R}^{5}$-equivalente a $x^{3}+x y^{3}$, que é 5 -determinado. Assim $g$ é $\mathcal{R}$-equivalente a $x^{3}+x y^{3}$. A

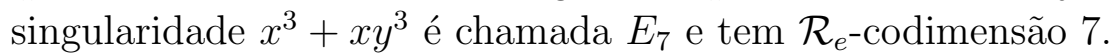

3. $a=b=0$. Em $J^{5}(2,1)$ consideramos os polinômios cujo 4-jato é $x^{3}$. Calculemos a transversal completa $T$ :

$$
m_{2}^{2}\left\langle x^{2}\right\rangle+T+m_{2}^{6} \supset m_{2}^{5}
$$

obtemos então que $T=\mathbb{R}\left\{x y^{4}, y^{5}\right\}$. Tal polinômio é $\mathcal{R}^{5}$-equivalente a $x^{3}+a x y^{4}+b y^{5}$. Temos então que se

(a) $b \neq 0$. Seja $N=\left\{p=x^{3}+a x y^{4}+y^{5} / a \in \mathbb{R}\right\} \subset J^{5}(2,1)$.

Temos que $\mathrm{N}$ é uma variedade conexa e $T_{p} N=\mathbb{R}\left\{x y^{4}\right\}, \forall p \in$ $N$. Além disso

$$
T_{p} G \cdot p=\frac{m_{2}\left\langle 3 x^{2}+a y^{4}, 4 a x y^{3}+5 y^{4}\right\rangle+m_{2}^{6}}{m_{2}^{6}}
$$

e $x y^{4} \in T_{p} G \cdot p$. E mais

$$
\begin{gathered}
\operatorname{dim} T_{p} G \cdot p=\operatorname{dim} \mathbb{R}\left\{x^{3}, x^{2} y, x^{4}, x^{3} y, x^{2} y^{2}, x^{5}, x^{4} y\right. \\
\left.x^{3} y^{2}, x^{2} y^{3}, x y^{4}, y^{5}\right\}=11
\end{gathered}
$$

Assim concluímos que $x^{3}+a x y^{4}+y^{5}$ é $\mathcal{R}^{5}$-equivalente a $x^{3}+y^{5}$, que é 5-determinado.

Portanto $g$ é $\mathcal{R}$-equivalente a $x^{3}+y^{5}$. A singularidade $x^{3}+y^{5}$ é chamada $E_{8}$ e tem $\mathcal{R}_{e^{-c o d i m e n s a ̃ o ~} 8 .}$

(b) $b=0$ e $a \neq 0$. Temos que $x^{3}+a x y^{4}$ é $\mathcal{R}^{5}$-equivalente a $x^{3} \pm x y^{4}$.

Em $J^{6}(2,1)$, consideramos os polinômios cujo 5-jato é $x^{3} \pm x y^{4}$. Calculamos novamente a transversal completa $T$ :

$$
m_{2}^{2}\left\langle 3 x^{2} \pm y^{4}, x y^{3}\right\rangle+T+m_{2}^{7} \supset m_{2}^{6}
$$

e obtemos que $T=\mathbb{R}\left\{y^{6}\right\}$. Assim os polinômios cujo 5-jato são $x^{3} \pm x y^{4}$, são $\mathcal{R}^{6}$-equivalente a $x^{3} \pm x y^{4}+a y^{6}$.

Na próxima seção concluiremos esta classificação introduzindo o conceito de germes simples. 


\subsection{Singularidades Simples}

Definição 2.4.1 Sejam $G$ um grupo de Lie agindo numa variedade $M$ e $x \in M$. Dizemos que uma órbita $G \cdot x$ é simples se existe uma vizinhança suficientemente pequena de $g_{0} \cdot x$ em $M$, para algum $g_{0} \in G$, que encontra somente um número finito de órbitas.

Proposição 2.4.2 Se tal vizinhança existe para $g_{0} \cdot x$, então existe para $g \cdot x$, $\forall g \in G$.

\section{Demonstração:}

Sejam $\phi: G \times M \rightarrow M, \phi(g, x)=g \cdot x$ a ação e $\phi_{g}: M \rightarrow M$ dada por $\phi_{g}(x)=g \cdot x$, além disso $\phi_{g}$ é difeomorfismo.

Temos que existe $V$ vizinhança de $g_{0} \cdot x$ tal que $V$ encontra um número finito de órbitas.

Para cada $g \in G$ seja $V^{\prime}=\phi_{g \cdot g_{0}^{-1}}(V)$. Temos que

$$
\phi_{g g_{0}^{-1}}\left(g_{0} \cdot x\right)=\left(g g_{0}^{-1}\right) \cdot\left(g_{0} \cdot x\right)=g \cdot x
$$

assim $V^{\prime}$ é uma vizinhança de $g \cdot x$. Além disso $\phi_{g g_{0}^{-1}}$ preserva órbitas. Portanto $V^{\prime}$ intercepta um número finito de órbitas.

Observação 2.4.3 Se a ação de $G$ em $M$ é algébrica então uma vizinhança de $g \cdot x$ encontra um número finito de órbitas ou uma família contínua.

Definição 2.4.4 Um germe $f \in m_{n}$ é simples se é $k$-determinado para algum $k$ e seu $k$-jato como um elemento de $J^{N}(n, 1), N>k$, tem uma vizinhança que encontra somente um número finito de $\mathcal{R}^{N}$ - órbitas.

\section{Observações 2.4.5}

1. Podemos substituir nas definições 2.4 .1 e 2.4 .4 vizinhança por transversal local.

2. Se $f \in m_{n}$ é simples, então $j^{k} f(0)$ é simples $\forall k \geq 1$. A recíproca é verdadeira para germes finitamente determinados.

3. Se a codimensão de $f$ é infinita então $f$ não é simples, pois $f$ não é finitamente determinado. 
Lema 2.4.6 Sejam $f, g \in m_{n}$. Se $j^{k} f(0)$ e $j^{k} g(0)$ não são $\mathcal{R}^{k}$-equivalentes em $J^{k}(n, 1)$, então $j^{N} f(0)$ e $j^{N} g(0)$ não são $\mathcal{R}^{N}$-equivalentes em $J^{N}(n, 1)$, para todo $N \geq k$.

\section{Demonstração:}

Suponhamos que $f$ e $g$ sejam $\mathcal{R}^{N}$-equivalentes em $J^{N}(n, 1), N \geq k$, isto é, existe $h:\left(\mathbb{R}^{n}, 0\right) \rightarrow\left(\mathbb{R}^{n}, 0\right)$ germe de difeomorfismo tal que

$$
j^{N}\left(j^{N} f(0) \circ j^{N} h(0)\right)=j^{N} g(0)
$$

assim

$$
j^{k} g(0)=j^{k}\left(j^{N} f(0) \circ j^{N} h(0)\right)=j^{k}\left(j^{k} f(0) \circ j^{k} h(0)\right)
$$

Logo $j^{k} f(0)$ e $j^{k} g(0)$ são $\mathcal{R}^{k}$-equivalentes em $J^{k}(n, 1)$, o que é uma contradição.

Proposição 2.4.7 Se $f \in m_{n}$ é simples, então corank $f \leq 2$.

\section{Demonstração:}

Suponha que $r=$ corank $f \geq 3$. Então pelo Splitting Lemma (2.1.3) temos que $f$ é $\mathcal{R}$-equivalente a $g\left(x_{1}, \ldots, x_{r}\right)+Q$, onde $g \in m_{r}^{3}$ e $Q$ é uma forma quadrática não degenerada nas variáveis $x_{r+1}, \ldots, x_{n}$.

Temos que $j^{2} g(0)=0$, assim $j^{3} g(0) \in H^{3}(r, 1)$. Notemos que a ação de $\mathcal{R}^{3}$ em $H^{3}(r, 1)$ é linear, isto é, coincide com a ação de $G l(r)$ em $H^{3}(r, 1)$. Além disso, como $r \geq 3$,

$$
\operatorname{dim} H^{3}(r, 1)=\left(\begin{array}{c}
r+2 \\
3
\end{array}\right)>\operatorname{dim} G l(r)=r^{2}
$$

A ação $\phi: G l(r) \times H^{3}(r, 1) \rightarrow H^{3}(r, 1)$ induz

$$
\phi_{j^{3} g(0)}: G l(r) \rightarrow G l(r) \cdot j^{3} g(0)
$$

Ainda,

$$
\operatorname{Im} d \phi_{j^{3} g(0)_{1}}=T_{j^{3} g(0)} G l(r) \cdot j^{3} g(0)
$$

daí

$$
\operatorname{dim} T_{j^{3} g(0)} G l(r) \cdot j^{3} g(0) \leq \operatorname{dim} G l(r)<\operatorname{dim} H^{3}(r, 1)
$$


Assim qualquer vizinhança de $j^{3} g(0)$ em $H^{3}(r, 1)$ intercepta infinitas órbitas.

Segue do Lema 2.4.6 que qualquer vizinhança de $j^{N} g(0)$ em $J^{N}(r, 1)$, $N \geq 3$, intercepta infinitas órbitas. Logo $g$ não é simples e portanto $f$ não é simples.

Seja $f \in m_{n}$ um germe de corank $\leq 2$ e codimensão finita. Se corank $f=1$, segue da Proposição 2.1.5 que $f$ é uma singularidade $A_{k-1}$, para algum $k$. Se corank $f=2$, vimos na seção 2.3 que $f$ é $\mathcal{R}$-equivalente a uma das seguintes singularidades:

1. $D_{k}: x^{2} y \pm y^{k-1} \pm x_{3}^{2} \pm \ldots \pm x_{n}^{2}, k \geq 4$

2. $E_{6}: x^{3} \pm y^{4} \pm x_{3}^{2} \pm \ldots \pm x_{n}^{2}$

3. $E_{7}: x^{3}+x y^{3} \pm x_{3}^{2} \pm \ldots \pm x_{n}^{2}$

4. $E_{8}: x^{3}+y^{5} \pm x_{3}^{2} \pm \ldots \pm x_{n}^{2}$

5. $g(x, y) \pm x_{3}^{2} \pm \ldots \pm x_{n}^{2}$ onde $j^{5} g(0)$ é $\mathcal{R}^{5}$-equivalente a $x^{3} \pm x y^{4}$. Temos que os polinômios em $J^{6}(2,1)$ cujo 5 -jato é $x^{3} \pm x y^{4}$ são $\mathcal{R}^{6}$-equivalentes a $h_{a}(x, y)=x^{3} \pm x y^{4}+a y^{6}$. Afirmamos que se $a \neq b$ então $h_{a}$ não é $\mathcal{R}^{6}$ equivalente a $h_{b}$. De fato, como a ação é algébrica temos um número finito ou uma família contínua de órbitas. Suponhamos que existe um número finito de órbitas, assim existe uma vizinhança $V$ de $a$ tal que se $b \in V \backslash\left\{a_{1}, \ldots, a_{r}\right\}$, então $h_{a}$ é $\mathcal{R}^{6}$-equivalente a $h_{b}$ em $J^{6}(2,1)$. Consideremos a curva $\gamma(t)=$ $x^{3}+x y^{4}+(a+t) y^{6}$ em $J^{6}(2,1)$. Então para $t$ próximo de zero, exceto para um número finito de $t^{\prime}$ 's, $\gamma(t) \in \mathcal{R}^{6} h_{a}$. Conseqüentemente $\gamma^{\prime}(0)=y^{6} \in T \mathcal{R}^{6} h_{a}$, o que é uma contradição (ver o final da Seção 2.3). Segue da Observação 2.4.5 (2) e do Lema 2.4.6 que $g$ não é simples. Portanto $f$ não é simples.

6. $g(x, y) \pm x_{3}^{2} \pm \ldots \pm x_{n}^{2}$ onde $j^{3} g(0)=0$. No que segue mostraremos que $g$ não é simples.

Lema 2.4.8 Existe um isomorfismo linear que leva três retas distintas de $\mathbb{R}^{2}$ que passam pela origem em três retas distintas de $\mathbb{R}^{2}$ que passam pela origem. 


\section{Demonstração:}

Sejam $r_{1}, r_{2}$ e $r_{3}\left(s_{1}, s_{2}\right.$ e $\left.s_{3}\right)$ três retas distintas em $\mathbb{R}^{2}$ passando pela origem. Sejam $v_{1}, v_{2}$ e $v_{3}\left(w_{1}, w_{2}\right.$ e $\left.w_{3}\right)$ os vetores diretores de $r_{1}, r_{2}$ e $r_{3}\left(s_{1}\right.$, $s_{2}$ e $\left.s_{3}\right)$ respectivamente.

Como $\left\{v_{1}, v_{2}\right\}$ é uma base de $\mathbb{R}^{2}$, existem $\alpha, \beta \in \mathbb{R}$ não nulos tais que $v_{3}=\alpha v_{1}+\beta v_{2}$. Analogamente existem $\gamma, \delta \in \mathbb{R}$ não nulos tais que $w_{3}=$ $\gamma w_{1}+\delta w_{2}$.

Definimos $T: \mathbb{R}^{2} \rightarrow \mathbb{R}^{2}$ por $T\left(v_{1}\right)=\frac{\gamma}{\alpha} w_{1}$ e $T\left(v_{2}\right)=\frac{\delta}{\beta} w_{2}$. Notemos que $T$ é um isomorfismo linear e

$$
T\left(v_{3}\right)=T\left(\alpha v_{1}+\beta v_{2}\right)=\alpha T\left(v_{1}\right)+\beta T\left(v_{2}\right)=\gamma w_{1}+\delta w_{2}=w_{3}
$$

Portanto, $T\left(r_{i}\right)=s_{i}, i=1,2,3$.

Corolário 2.4.9 Seja $p(x, y)=\left(a_{1} x+b_{1} y\right)\left(a_{2} x+b_{2} y\right)\left(a_{3} x+b_{3} y\right)\left(a_{4} x+b_{4} y\right)$ um polinômio tal que $p(x, y)=0$ define 4 retas distintas em $\mathbb{R}^{2}$. Então existe um isomorfismo linear $T: \mathbb{R}^{2} \rightarrow \mathbb{R}^{2}$ que leva estas 4 retas nas 4 retas dadas por $q(x, y)=x y(x+y)(x-\lambda y)=0$, para algum $\lambda \in \mathbb{R}$.

\section{Demonstração:}

Consideremos a reta $r_{i}$ dada pela equação $r_{i}: a_{i} x+b_{i} y=0, i=1,2,3,4$. Sejam $s_{1}$ a reta $x=0, s_{2}$ a reta $y=0$ e $s_{3}$ a reta $x+y=0$.

Pelo Lema 2.4.8 existe um isomorfismo linear $T: \mathbb{R}^{2} \rightarrow \mathbb{R}^{2}$ tal que $T\left(r_{i}\right)=$ $s_{i}, i=1,2,3$. Assim, $T\left(r_{4}\right)$ é uma reta da equação $x-\lambda y=0$ para algum $\lambda \in \mathbb{R}$.

Sejam $g \in m_{2}$ tal que $j^{3} g(0)=0$ e $U$ uma vizinhança de $j^{4} g(0)$ em $J^{4}(2,1)$. Considerando polinômios complexos, existe um elemento em $U$ da forma $p(x, y)=\left(a_{1} x+b_{1} y\right)\left(a_{2} x+b_{2} y\right)\left(a_{3} x+b_{3} y\right)\left(a_{4} x+b_{4} y\right)$ tal que $p(x, y)=0$ define quatro retas distintas em $\mathbb{C}^{2}$. Seja $q(x, y)=x y(x+y)(x-\lambda y)$ dado pelo Corolário 2.4.9.

Definimos $V(p)=\left\{(x, y) \in \mathbb{C}^{2} / p(x, y)=0\right\}$. Então $V(p) \subset V(q \circ T)$. Consideremos um conjunto $X \subset \mathbb{C}^{2}$ e o ideal $I(X)=\{p \in \mathbb{C}[x, y] / p(\bar{x}, \bar{y})=$ $0, \forall(\bar{x}, \bar{y}) \in X\}$. Então $I(V(p)) \supset I(V(q \circ T))$. Mas $I(V(p))=(p)$ e $I(V(q \circ$ $T))=(q \circ T)(\operatorname{ver}[11]$, p. 54), onde $(p)$ denota o ideal gerado por $p$ em $\mathbb{C}[x, y]$. Logo existe $h \in \mathbb{C}[x, y]$ tal que $q \circ T=h p$. 
Usando o argumento acima para $p \circ T^{-1}$ e $q$ temos que existe $k \in \mathbb{C}[x, y]$ tal que $p \circ T^{-1}=k q$.

Assim,

$$
q \circ T=h p=h(k \circ T)(q \circ T)
$$

daí,

$$
q \circ T(1-h(k \circ T))=0
$$

Como $q \circ T \neq 0$ temos que $h(k \circ T)=1$, isto é, $h k$ é constante. Logo $h$ e $k$ são constantes não nulas.

Conseqüentemente $p$ e $q$ são linearmente equivalentes como polinômios complexos.

Mas $q$ não é simples. De fato, seja

$$
f_{t}(x, y)=x y(x+y)(x-t y)
$$

Para $t \in \mathbb{C}$, o conjunto de zeros de $f_{t}$ é constituído de quatro retas em $\mathbb{C}^{2}$. Tomemos $z_{i}(t)=\frac{\alpha_{i}}{\beta_{i}}$ em $\mathbb{C}_{\infty}$ (ver[3], p. 8), onde $\alpha_{i}$ e $\beta_{i}, i=1,2,3,4$, são os coeficientes de $x$ e $y$ respectivamente em $x=0, y=0, x+y=0, x-t y=0$. Temos que o cross ratio destas quatro retas é dado por

$$
\frac{\left(z_{3}(t)-z_{1}(t)\right)\left(z_{4}(t)-z_{2}(t)\right)}{\left(z_{4}(t)-z_{1}(t)\right)\left(z_{3}(t)-z_{2}(t)\right)}
$$

e é denotado por $\left(z_{1}(t), z_{2}(t), z_{3}(t), z_{4}(t)\right)$. Ao permutarmos estas quatro retas obtemos os seguintes valores para o cross ratio:

$$
-t,-\frac{1}{t}, 1+t, \frac{1}{1+t}, \frac{t}{1+t}, \frac{1+t}{t}
$$

Suponhamos que para $t_{1} \neq t_{2}$ existe um isomorfismo linear $T: \mathbb{C}^{2} \rightarrow \mathbb{C}^{2}$ tal que $f_{t_{1}} \circ T=f_{t_{2}}, t_{1}, t_{2} \in \mathbb{C}$.

Como o cross ratio é invariante por automorfismo (ver [3], p. 48), temos então que

$$
\left(z_{1}\left(t_{1}\right), z_{2}\left(t_{1}\right), z_{3}\left(t_{1}\right), z_{4}\left(t_{1}\right)\right)=\left(z_{1}\left(t_{2}\right), z_{2}\left(t_{2}\right), z_{3}\left(t_{2}\right), z_{4}\left(t_{2}\right)\right)
$$

mas isso só ocorre se $t_{1}=t_{2}$.

Portanto $f_{t_{1}}$ e $f_{t_{2}}$ não são $\mathcal{R}^{4}$-equivalentes em $\mathbb{C}$ se $t_{1} \neq t_{2}$. Com isso $U$ intercepta infinitas órbitas em $J^{4}(2,1)$ em $\mathbb{C}$. Observemos que dois elemetos 
$f_{t_{1}}$ e $f_{t_{2}}$ podem não ser equivalentes em $\mathbb{R}$ mas serem em $\mathbb{C}$, o contrário não ocorre pois $\mathbb{R}$ é um caso particular de $\mathbb{C}$. Com isso concluímos que $U$ intercepta infinitas órbitas em $J^{4}(2,1)$ em $\mathbb{R}$. Portanto $g$ não é simples.

No que segue vamos mostrar que as singularidades $A_{k}, D_{k}, E_{6}, E_{7}$ e $E_{8}$ são simples. Como já sabemos que tais singularidades são finitamente determinadas, resta mostrar que seu $k$-jato, para $k$ grande, como um elemento de $J^{r}(n, 1), r \geq k$, tem uma transversal que intercepta um número finito de $\mathcal{R}^{n}$-órbitas (ver Definição 2.4.4). Para isso precisamos do seguinte resultado:

Proposição 2.4.10 Como transversais às órbitas das singularidades $A_{k}$, $D_{k}, E_{6}, E_{7}$ e $E_{8}$ no espaço $J^{r}(n, 1)$, com $r \geq k+1, r \geq k-1, r \geq 4$, $r \geq 4$ e $r \geq 5$ respectivamente, podemos tomar os seguintes subconjuntos de $J^{r}(n, 1)$ :

$A_{k}:\left\{ \pm x^{k+1} \pm y^{2}+Q+\varepsilon_{2} x^{2}+\varepsilon_{3} x^{3}+\ldots+\varepsilon_{k} x^{k}, \varepsilon_{1}, \ldots, \varepsilon_{k} \in \mathbb{R}\right\} ;$

$D_{k}:\left\{x^{2} y \pm y^{k-1}+Q+\varepsilon_{2} y^{2}+\ldots+\varepsilon_{k-2} y^{k-2}+\varepsilon_{k-1} x y+\varepsilon_{k} x^{2}, \varepsilon_{1}, \ldots, \varepsilon_{k} \in \mathbb{R}\right\} ;$

$E_{6}:\left\{x^{3} \pm y^{4}+Q+\varepsilon_{2} y^{2}+\varepsilon_{3} y^{3}+\varepsilon_{4} x^{2}+\varepsilon_{5} x y+\varepsilon_{6} x y^{2}, \varepsilon_{1}, \ldots, \varepsilon_{6} \in \mathbb{R}\right\} ;$

$E_{7}:\left\{x^{3}+x y^{3}+Q+\varepsilon_{2} y^{2}+\varepsilon_{3} y^{3}+\varepsilon_{4} y^{4}+\varepsilon_{5} x^{2}+\varepsilon_{6} x y+\varepsilon_{7} x y^{2}, \varepsilon_{1}, \ldots, \varepsilon_{7} \in \mathbb{R}\right\} ;$

$E_{8}:\left\{x^{3}+y^{5}+Q+\varepsilon_{2} y^{2}+\varepsilon_{3} y^{3}+\varepsilon_{4} y^{4}+\varepsilon_{5} x^{2}+\varepsilon_{6} x y+\varepsilon_{7} x y^{2}+\varepsilon_{8} x y^{3}, \varepsilon_{1}, \ldots, \varepsilon_{8} \in \mathbb{R}\right\}$;

onde $Q$ denota a forma quadrática definido por

$$
Q\left(x_{3}, \ldots, x_{n}\right)= \pm x_{3}^{2} \pm \ldots \pm x_{n}^{2}
$$

\section{Demonstração:}

Pela Seção 2.3, basta considerarmos os casos em que as séries são

$$
\begin{array}{ll}
A_{k}: & \pm x^{k+1} \pm y^{2} \\
D_{k}: & x^{2} y \pm y^{k-1} \\
E_{6}: & x^{3} \pm y^{4} \\
E_{7}: & x^{3}+x y^{3} \\
E_{8}: & x^{3}+y^{5}
\end{array}
$$

Denotemos por $g(x, y)$ uma destas singularidades. Queremos encontrar um subespaço $\mathbb{T}$ de $J^{r}(2,1)$ transversal à órbita de $j^{r} g(0)$. Assim, $\mathbb{T}$ deve satisfazer:

$$
T \mathcal{R}^{r} j^{r} g(0)+T_{j^{r} g(0)} \mathbb{T}=J^{r}(2,1)
$$


ou seja

$$
\frac{m_{2} J g+m_{2}^{r+1}}{m_{2}^{r+1}}+\mathbb{T}=J^{r}(2,1)
$$

Como todo elemento em $J^{r}(2,1)$ não nulo é jato de submersão, basta encontrarmos $\mathbb{T}$ satisfazendo

$$
m_{2} J g+m_{2}^{r+1}+\mathbb{T} \supset m_{2}^{r}
$$

Dessa forma obtemos os seguintes $\mathbb{T}^{\prime}$ s para cada caso:

$$
\begin{array}{ll}
A_{k}: & \mathbb{T}=\left\{x^{2}, x^{3}, \ldots, x^{k}\right\} \\
D_{k}: & \mathbb{T}=\left\{x^{2}, y^{2}, y^{3}, \ldots, y^{k-2}, x y\right\} \\
E_{6}: & \mathbb{T}=\left\{x^{2}, y^{2}, y^{3}, x y, x y^{2}\right\} \\
E_{7}: & \mathbb{T}=\left\{x^{2}, y^{2}, y^{3}, y^{4}, x y, x y^{2}\right\} \\
E_{8}: & \mathbb{T}=\left\{x^{2}, x y, y^{2}, y^{3}, y^{4}, x y, x y^{2}, x y^{3}\right\}
\end{array}
$$

Corolário 2.4.11 As singularidades $A_{k}, D_{k}, E_{6}, E_{7}$ e $E_{8}$ são simples.

\section{Demonstração:}

Mostremos que as transversais descritas na Proposição 2.4.10 interceptam finitas órbitas.

- No caso $A_{k}$ temos que a transversal é da seguinte forma

$$
g\left(x, y, x_{3}, \ldots, x_{n}\right)= \pm x^{k+1} \pm y^{2}+Q+\varepsilon_{2} x^{2}+\varepsilon_{3} x^{3}+\ldots+\varepsilon_{k} x^{k}
$$

Observemos que se $\varepsilon_{i} \neq 0$ para algum $i=2, \ldots, n$ e $\varepsilon_{j}=0, \forall j<i$, temos que $g$ é uma singularidade $A_{i-1}$. Obtemos então que a transversal intercepta unicamente as órbitas $A_{j}, j=1, \ldots, k-1$.

- No caso $D_{k}$ temos que a transversal é dada por

$$
\left(x, y, x_{3}, \ldots, x_{n}\right) \mapsto x^{2} y \pm y^{k-1}+Q+\varepsilon_{2} y^{2}+\ldots+\varepsilon_{k-2} y^{k-2}+\varepsilon_{k-1} x y+\varepsilon_{k} x^{2}
$$

É suficiente considerarmos

$$
g(x, y)=x^{2} y \pm y^{k-1}+\varepsilon_{2} y^{2}+\ldots+\varepsilon_{k-2} y^{k-2}+\varepsilon_{k-1} x y+\varepsilon_{k} x^{2}
$$


1. se $\varepsilon_{k-1}^{2} \neq 4 \varepsilon_{2} \varepsilon_{k}$ temos que $g$ é Morse

2. Suponhamos $\varepsilon_{2}, \varepsilon_{k-1}, \varepsilon_{k}$ não todos nulos tais que $\varepsilon_{k-1}^{2}=4 \varepsilon_{2} \varepsilon_{k}$. Afirmamos que $g$ é $k$-1-determinado. Primeiro mostremos nos itens (a) e (b) seguintes que $m_{2}^{k-1} \subset m_{2} J g+m_{2}^{k}$. Logo $g$ é $k-1$-determinado, pela Observação 1.5.3(2).

(a) Se $\varepsilon_{2} \neq 0$ e $\varepsilon_{k-1}=\varepsilon_{k}=0$ temos que

$$
m_{2} J g=m_{2}\left\langle x y, x^{2} \pm(k-1) y^{k-2}+2 \varepsilon_{2} y+\ldots+(k-2) \varepsilon_{k-2} y^{k-3}\right\rangle
$$

Logo $x^{i} y^{j} \in m_{2} J g+m_{2}^{k}$ onde $i, j \neq 0$ e $2 \leq i+j \leq k-1$ e portanto $x^{k-1}, y^{k-1} \in m_{2} J g+m_{2}^{k}$.

(b) Se $\varepsilon_{2}=\varepsilon_{k-1}=0$ e $\varepsilon_{k} \neq 0$ temos que

$$
\begin{gathered}
m_{2} J g=m_{2}\left\langle 2 x y+2 \varepsilon_{k} x, x^{2} \pm(k-1) y^{k-2}+3 \varepsilon_{3} y^{2}+\ldots\right. \\
\left.+(k-2) \varepsilon_{k-2} y^{k-3}\right\rangle
\end{gathered}
$$

Assim $x^{i} y^{j} \in m_{2} J g+m_{2}^{k}$ onde $i \neq 0$ e $i+j=k-1$, e dessa forma $y^{k-1} \in m_{2} J g+m_{2}^{k}$.

(c) Se $\varepsilon_{2}, \varepsilon_{k}, \varepsilon_{k-1}$ são todos não nulos. Neste caso seja $h(x, y)=$ $g \circ T(x, y)$ onde

$$
T(x, y)=\left(\frac{\varepsilon_{k-1}}{2 \varepsilon_{k}} y, x-y\right)
$$

Como $m_{2} J h=m_{2}\left\langle\varepsilon_{k-1} x+\frac{\varepsilon_{k-1}}{\varepsilon_{k}}\left(x y-y^{2}\right), \frac{\partial}{\partial y} h(x, y)\right\rangle$ assim temos $x^{i} y^{j} \in m_{2} J h+m_{2}^{k}, i \neq 0$ e $2 \leq i+j \leq k-1$, logo $y^{k-1} \in m_{2} J h+m_{2}^{k}$. Como $g$ e $h$ são $\mathcal{R}$-equivalentes e $h$ é $(k-1)$-determinado, segue da Proposição 1.5.5 que $g$ é $(k-1)$-determinado.

Segue do Splitting Lemma (2.1.3) que $g$ é $\mathcal{R}$-equivalente a $\phi(x)+y^{2}$, onde $\phi \in m_{1}^{3}$. Segue da Proposição 1.5.5 que $\phi(x)+y^{2}$ é $(k-1)$ determinado, isto é, $\phi(x)+y^{2}$ é uma singularidade $A_{j}, j \leq k-2$. Portanto $g$ é uma singularidade $A_{j}$ com $j \leq k-2$. 
3. Se $\varepsilon_{2}=\varepsilon_{k-1}=\varepsilon_{k}=0$. Seja $j$ tal que $\varepsilon_{j} \neq 0$ e $\varepsilon_{i}=0$ para todo $i<j$. Assim,

$$
g(x, y)=x^{2} y \pm y^{k-1}+\varepsilon_{j} y^{j}+\ldots+\varepsilon_{k-2} y^{k-2}
$$

Temos pela Seção 2.3 que $g$ é $\mathcal{R}$-equivalente a $y^{j} \pm x^{2} y, 3 \leq j \leq k-2$, que é uma singularidade $D_{j+1}$.

- No caso $E_{6}$. Seja

$$
g(x, y)=x^{3} \pm y^{4}+\varepsilon_{2} y^{2}+\varepsilon_{3} y^{3}+\varepsilon_{4} x^{2}+\varepsilon_{5} x y+\varepsilon_{6} x y^{2}
$$

Suponhamos $\varepsilon_{2}, \varepsilon_{4}, \varepsilon_{5}$ todos não nulos: se $\varepsilon_{5}^{2} \neq 4 \varepsilon_{2} \varepsilon_{4}, g$ é Morse e se $\varepsilon_{5}^{2}=4 \varepsilon_{2} \varepsilon_{4}$, analogamente ao caso $D_{k}$, obtemos que $g$ é 3 -determinado e portanto $g$ é $\mathcal{R}$-equivalente a $\phi(x)+y^{2}$, onde $\phi(x) \in m_{1}^{3}$. Como $\phi(x)+y^{2}$ é 3-determinado, segue que $\phi(x)$ é uma singularidade $A_{j}, j \leq 2$. Portanto $g$ é uma singularidade $A_{j}$ com $j \leq 2$.

Se $\varepsilon_{2}=\varepsilon_{4}=\varepsilon_{5}=0$ temos

$$
g(x, y)=x^{3} \pm y^{4}+\varepsilon_{3} y^{3}+\varepsilon_{6} x y^{2}
$$

onde $\varepsilon_{3} \neq 0$ ou $\varepsilon_{6} \neq 0$. Notemos que $j^{3} g(0)=x^{3}+\varepsilon_{3} y^{3}+\varepsilon_{6} x y^{2}$. Segue da Proposição 2.3.1 que $j^{3} g(0)$ é $\mathcal{R}^{3}$-equivalente a $x^{2} y+y^{3}$. Como este último é 3-determinado temos que $g$ é $\mathcal{R}$-equivalente a $x^{2} y+y^{3}$, que é uma singularidade $D_{4}$.

- Caso $E_{7}$. Seja

$$
g(x, y)=x^{3}+x y^{3}+\varepsilon_{2} y^{2}+\varepsilon_{3} y^{3}+\varepsilon_{4} y^{4}+\varepsilon_{5} x^{2}+\varepsilon_{6} x y+\varepsilon_{7} x y^{2}
$$

Suponhamos que $\varepsilon_{2}, \varepsilon_{5}, \varepsilon_{6}$ sejam não nulos: se $\varepsilon_{6}^{2} \neq 4 \varepsilon_{2} \varepsilon_{5}$ temos que $g$ é Morse e se $\varepsilon_{6}^{2}=4 \varepsilon_{2} \varepsilon_{5}$. Segue analogamente ao caso $E_{6}$ que $g$ é uma singularidade $A_{j} \operatorname{com} j \leq 3$.

Se $\varepsilon_{2}=\varepsilon_{5}=\varepsilon_{6}=0$ e $\varepsilon_{3} \neq 0$ ou $\varepsilon_{7} \neq 0$, segue analogamente ao caso $E_{6}$ que $g$ é uma singularidade $D_{4}$.

Se $\varepsilon_{2}=\varepsilon_{3}=\varepsilon_{5}=\varepsilon_{6}=\varepsilon_{7}=0$ e $\varepsilon_{4} \neq 0$, temos

$$
g(x, y)=x^{3}+x y^{3}+\varepsilon_{4} y^{4}
$$

Logo $g$ é $\mathcal{R}$-equivalente a $x^{3}+a x y^{3} \pm y^{4}$. 
Como $j^{3} g(0)=x^{3}$, segue da Seção 2.3, que $x^{3}+a x y^{3} \pm y^{4}$ é $\mathcal{R}$-equivalente a $x^{3} \pm y^{4}$, que é uma singularidade $E_{6}$.

- Caso $E_{8}$. Temos

$$
g(x, y)=x^{3}+y^{5}+\varepsilon_{2} y^{2}+\varepsilon_{3} y^{3}+\varepsilon_{4} y^{4}+\varepsilon_{5} x^{2}+\varepsilon_{6} x y+\varepsilon_{7} x y^{2}+\varepsilon_{8} x y^{3}
$$

Suponhamos que $\varepsilon_{2}, \varepsilon_{5}, \varepsilon_{6}$ sejam não nulos: se $\varepsilon_{6}^{2} \neq 4 \varepsilon_{2} \varepsilon_{5}$ temos que $g$ é Morse e se $\varepsilon_{6}^{2}=4 \varepsilon_{2} \varepsilon_{5}$, segue analogamente ao caso $E_{6}$ que $g$ é uma singularidade $A_{j}$ com $j \leq 4$.

Se $\varepsilon_{2}=\varepsilon_{5}=\varepsilon_{6}=0$ e $\varepsilon_{3} \neq 0$ ou $\varepsilon_{7} \neq 0$, segue analogamente ao caso $E_{6}$ que $g$ é uma singularidade $D_{4}$.

Se $\varepsilon_{2}=\varepsilon_{3}=\varepsilon_{5}=\varepsilon_{6}=\varepsilon_{7}=0$ e $\varepsilon_{4} \neq 0$ ou $\varepsilon_{8} \neq 0$, temos

$$
g(x, y)=x^{3}+y^{5}+\varepsilon_{4} y^{4}+\varepsilon_{8} x y^{3}
$$

e

$$
j^{4} g(0)=x^{3}+\varepsilon_{4} y^{4}+\varepsilon_{8} x y^{3}
$$

Observemos que

1. se $\varepsilon_{4} \neq 0$ e $\varepsilon_{8}=0$. Notemos que tomando $T(x, y)=\left(x, \frac{1}{\left(\varepsilon_{4}\right)^{1 / 4}} y\right)$, se $\varepsilon_{4}>0$, e $T(x, y)=\left(x, \frac{1}{\left(-\varepsilon_{4}\right)^{1 / 4}} y\right)$, se $\varepsilon_{4}<0$ obtemos que $j^{4} g(0) \circ$ $T(x, y)=x^{3} \pm y^{4}$, como este é 4-determinado, concluímos que $g$ é $\mathcal{R}$-equivalente a $x^{3} \pm y^{4}$, que é uma singularidade $E_{6}$.

2. se $\varepsilon_{4}=0$ e $\varepsilon_{8} \neq 0$. Notemos que tomando $T(x, y)=\left(x, \frac{1}{\left(\varepsilon_{8}\right)^{1 / 3}} y\right)$ temos que $j^{4} g(0) \circ T(x, y)=x^{3}+x y^{3}$, pela Seção 2.3 temos que este é $\mathcal{R}^{5}$-equivalente a $x^{3}+x y^{3}$, sendo que este último é 5 -determinado. Conseqüentemente $g$ é $\mathcal{R}$-equivalente a $x^{3}+x y^{3}$, que é uma singularidade $E_{7}$.

3. se $\varepsilon_{4} \neq 0$ e $\varepsilon_{8} \neq 0$. Pela seção 2.3 , temos que $j^{4} g(0)$ é $\mathcal{R}^{4}$-equivalente a $x^{3} \pm y^{4}$, como este último é 4-determinado, concluímos que $g$ é $\mathcal{R}$ equivalente a $x^{3} \pm y^{4}$, que é uma singularidade $E_{6}$.

Esta seção consiste na demonstração do seguinte resultado: 
Teorema 2.4.12 (Arnol'd) Seja $f \in m_{n}$. Então $f$ é simples se, e somente se, é $\mathcal{R}$-equivalente a um dos seguintes:

$$
\begin{array}{llll}
A_{k}: & f\left(x, y, x_{3}, \ldots, x_{n}\right)= \pm x^{k+1} \pm y^{2}+Q & , \mathcal{R}_{e}-\operatorname{cod} f=k-1 & , k \geq 1 \\
D_{k}: & f\left(x, y, x_{3}, \ldots, x_{n}\right)=x^{2} y \pm y^{k-1}+Q & , \mathcal{R}_{e}-\operatorname{cod} f=k-1 & , k \geq 4 \\
E_{6}: & f\left(x, y, x_{3}, \ldots, x_{n}\right)=x^{3} \pm y^{4}+Q & , \mathcal{R}_{e}-\operatorname{cod} f=5 & \\
E_{7}: & f\left(x, y, x_{3}, \ldots, x_{n}\right)=x^{3}+x y^{3}+Q & , \mathcal{R}_{e}-\operatorname{cod} f=6 \\
E_{8}: & f\left(x, y, x_{3}, \ldots, x_{n}\right)=x^{3}+y^{5}+Q & , \mathcal{R}_{e}-\operatorname{cod} f=7
\end{array}
$$

onde $Q$ é a forma quadrática definida por

$$
Q\left(x_{3}, \ldots, x_{n}\right)= \pm x_{3}^{2} \pm \ldots \pm x_{n}^{2} .
$$




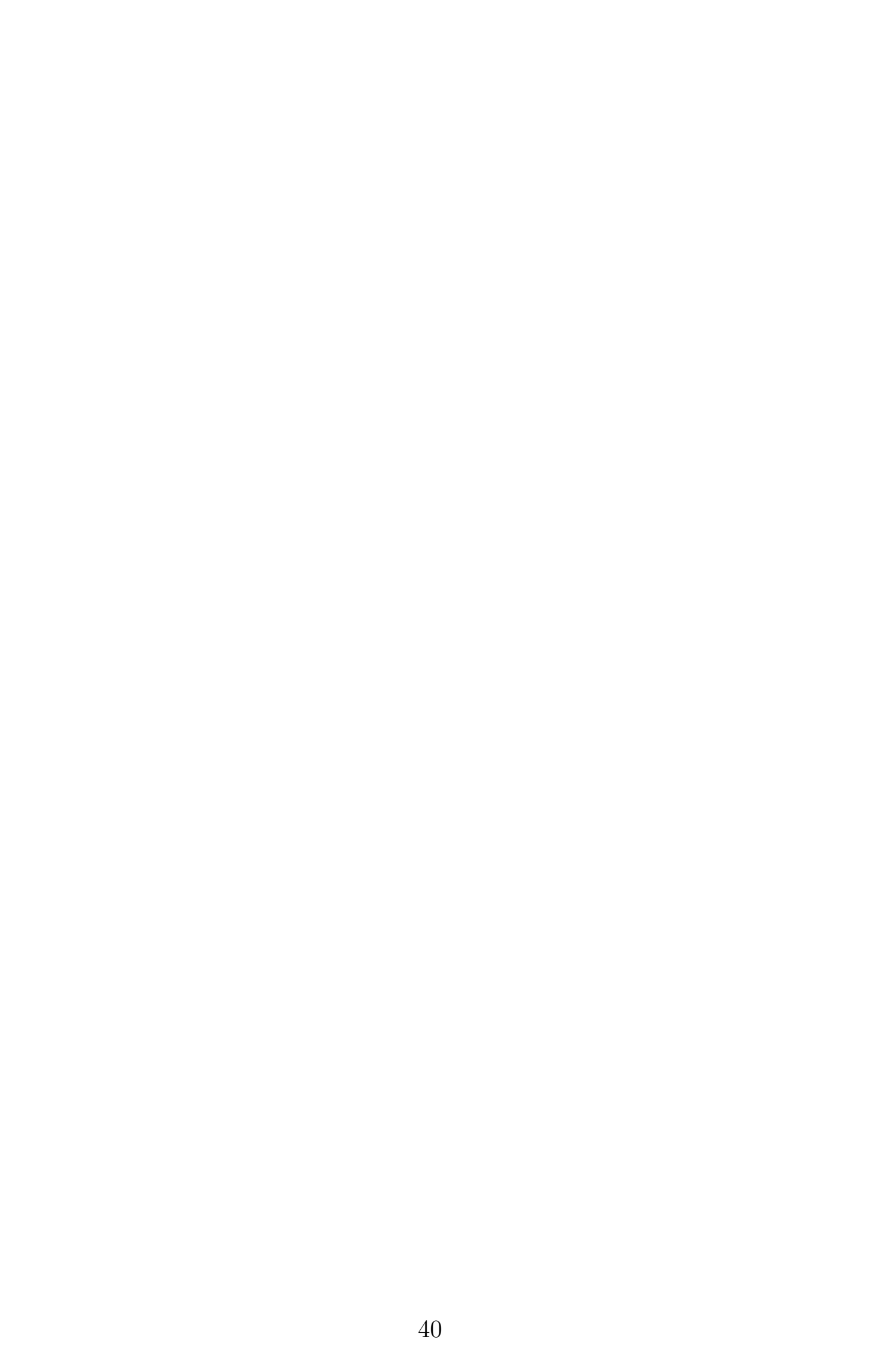




\section{Capítulo 3}

\section{Classificação dos germes $\left(\mathbb{R}^{2}, 0\right) \rightarrow\left(\mathbb{R}^{2}, 0\right)$}

Neste capítulo apresentamos a classificação dos germes $\left(\mathbb{R}^{2}, 0\right) \rightarrow\left(\mathbb{R}^{2}, 0\right)$ de corank 1 e $\mathcal{A}$-codimensão $\leq 4$ através do Método da Transversal Completa.

Utilizamos o software "Transversal" [10] para o cálculo de algumas codimensões e transversais completas.

\section{$3.1 \quad$ O grupo $\mathcal{A}$}

Denotemos o conjunto dos germes $\left(\mathbb{R}^{n}, 0\right) \rightarrow \mathbb{R}^{p}$ por $\varepsilon_{n, p}$. Quando $p=1$ denotamos simplesmente por $\varepsilon_{n}$. Temos que $\varepsilon_{n, p}$ é um $\varepsilon_{n}$-módulo.

Seja $D_{n}$, o grupo constituído dos germes de difeomorfismos de $\left(\mathbb{R}^{n}, 0\right) \rightarrow$ $\left(\mathbb{R}^{n}, 0\right)$ com a operação de composição. O grupo $D_{n} \times D_{p}$ age em $m_{n} \cdot \varepsilon_{n, p}$ da seguinte maneira

$$
(H, K) \cdot f=K \circ f \circ H^{-1}
$$

onde $(H, K) \in D_{n} \times D_{p}$ e $f \in m_{n} \cdot \varepsilon_{n, p}$. O grupo $D_{n} \times D_{p}$ com esta ação é denotado por $\mathcal{A}$.

O espaço tangente à órbita de $f \in m_{n} \cdot \varepsilon_{n, p}$ segundo o grupo $\mathcal{A}$ é dado por

$$
T \mathcal{A} \cdot f=m_{n}\left\{\frac{\partial f}{\partial x_{1}}, \ldots, \frac{\partial f}{\partial x_{n}}\right\}+f^{*} m_{p} \cdot\left\{e_{1}, \ldots, e_{p}\right\}
$$


onde $e_{1}, \ldots, e_{p}$ são elementos da base canônica de $\mathbb{R}^{p}$, considerados como elementos de $\varepsilon_{n, p}$. O conjunto $m_{n}\left\{\frac{\partial f}{\partial x_{1}}, \ldots, \frac{\partial f}{\partial x_{n}}\right\}$ é um $\varepsilon_{n}$-submódulo gerado por $\frac{\partial f}{\partial x_{1}}, \ldots, \frac{\partial f}{\partial x_{n}}$ com coeficientes em $m_{n}$ e $f^{*} m_{p} \cdot\left\{e_{1}, \ldots, e_{p}\right\}$ é gerado por $e_{1}, \ldots, e_{p}$ com coeficientes em $f^{*} m_{p}=\left\{g \circ f / g \in m_{p}\right\}$.

$\mathrm{O}$ espaço tangente estendido à órbita de $f \in m_{n} \cdot \varepsilon_{n, p}$ segundo o grupo $\mathcal{A}$ é dado por

$$
T \mathcal{A}_{e} \cdot f=\varepsilon_{n}\left\{\frac{\partial f}{\partial x_{1}}, \ldots, \frac{\partial f}{\partial x_{n}}\right\}+f^{*} \varepsilon_{p} \cdot\left\{e_{1}, \ldots, e_{p}\right\}
$$

Definição 3.1.1 A $\mathcal{A}$-codimensão de $f$ é definida por

$$
\mathcal{A}-\operatorname{cod} f=\operatorname{dim} \frac{m_{n} \cdot \varepsilon_{n, p}}{T \mathcal{A} \cdot f}
$$

e a $\mathcal{A}_{e}$-codimensão de $f$ por

$$
\mathcal{A}_{e}-\operatorname{cod} f=\operatorname{dim} \frac{\varepsilon_{n, p}}{T \mathcal{A}_{e} \cdot f}
$$

Teorema 3.1.2 Seja $f \in m_{2} \cdot \varepsilon_{2,2}$. Se $\mathcal{A}_{e}-\operatorname{cod} f>0$ então temos a seguinte relação

$$
\mathcal{A}-\operatorname{cod} f=\mathcal{A}_{e} \operatorname{cod} f-2
$$

\section{Demonstração:}

Ver [8].

Seja $H^{k+1}(n, p)$ o subespaço de $J^{k+1}(n, p)$ constituído dos jatos cujos componentes são polinômios homogêneos de grau $k+1$. Seja $\mathcal{A}_{1}$ o subgrupo de $\mathcal{A}$ cujo os elementos têm 1 -jato igual à identidade. Seja $\mathcal{A}_{1}^{k}$ o espaço dos $k$-jatos dos elementos de $\mathcal{A}_{1}$. O espaço tangente a $f \in m_{n} \varepsilon_{n, p}$ segundo o grupo $\mathcal{A}_{1}$ é dado por

$$
T \mathcal{A}_{1} \cdot f=m_{n}^{2}\left\{\frac{\partial f}{\partial x_{1}}, \ldots, \frac{\partial f}{\partial x_{n}}\right\}+f^{*} m_{p}^{2}\left\{e_{1}, \ldots, e_{p}\right\}
$$

O resultado seguinte é conseqüência da Proposição 2.2.1. 
Proposição 3.1.3 (Transversal Completa para jatos no grupo $\mathcal{A}$ ) Sejam $f \in J^{k}(n, p)$ e $T \subset H^{k+1}(n, p)$ um subespaço tais que

$$
T \mathcal{A}_{1} \cdot f+T+m_{n}^{k+2} \cdot \varepsilon_{n, p} \supset m_{n}^{k+1} \cdot \varepsilon_{n, p}
$$

Então qualquer $g \in J^{k+1}(n, p)$ com $j^{k} g(0)=f$ é $\mathcal{A}_{1}^{k}$-equivalente a $f+\beta$, onde $\beta \in T$.

Definição 3.1.4 Um germe $f \in m_{n} \cdot \varepsilon_{n, p}$ é dito $k$-A-determinado se qualquer $g \in m_{n} \cdot \varepsilon_{n, p}$ tal que $j^{k} g(0)=j^{k} f(0)$ é $\mathcal{A}$-equivalente a $f$. E mais, $f$ é dito finitamente $\mathcal{A}$-determinado se é $k$-A-determinado para algum $k$.

Teorema 3.1.5 Se

$$
\begin{aligned}
& \text { 1. } m_{n}^{l} \cdot \varepsilon_{n, p} \subset \varepsilon_{n}\left\{\frac{\partial f}{\partial x_{1}}, \ldots, \frac{\partial f}{\partial x_{n}}\right\}+f^{*} m_{p} \cdot \varepsilon_{p}\left\{e_{1}, \ldots, e_{p}\right\} \\
& \text { 2. } m_{n}^{k} \cdot \varepsilon_{n, p} \subset \varepsilon_{n}\left\{\frac{\partial f}{\partial x_{1}}, \ldots, \frac{\partial f}{\partial x_{n}}\right\}+f^{*} \varepsilon_{p}\left\{e_{1}, \ldots, e_{p}\right\}
\end{aligned}
$$

então $f$ é $(l+k)$-A-determinado.

\section{Demonstração:}

Ver [9].

\subsection{A Classificação}

Seja $F:\left(\mathbb{R}^{2}, 0\right) \rightarrow\left(\mathbb{R}^{2}, 0\right)$ um germe de uma aplicação de classe $C^{\infty}$, dado por $F(x, y)=\left(f_{1}(x, y), f_{2}(x, y)\right)$.

Aqui definimos o rank de $F$ como sendo o rank da matriz

$$
\left(\begin{array}{ll}
\frac{\partial f_{1}}{\partial x}(0,0) & \frac{\partial f_{1}}{\partial y}(0,0) \\
\frac{\partial f_{2}}{\partial x}(0,0) & \frac{\partial f_{2}}{\partial y}(0,0)
\end{array}\right)
$$


e o corank de $F=2$ - rank de $F$.

Se $F$ tem corank zero, temos que $F$ é uma submersão e pela Forma Local das Submersões (1.1.3), temos que existe um difeomorfismo $H$ tal que $F \circ H^{-1}(x, y)=(x, y)$.

Nosso objetivo é classificar os germes de corank 1 e $\mathcal{A}$-codimensão $\leq 4$ usando o método da Transversal Completa (3.1.3).

Primeiramente vejamos a seguinte

Proposição 3.2.1 Seja $F \in m_{2} \cdot \varepsilon_{2,2}$ de corank 1. Então $F$ é $\mathcal{A}$-equivalente $a(x, y) \mapsto(x, \bar{F}(x, y))$.

\section{Demonstração:}

Suponhamos $F=\left(f_{1}, f_{2}\right)$. Temos que

$$
\operatorname{det}\left(\begin{array}{ll}
\frac{\partial f_{1}}{\partial x}(0,0) & \frac{\partial f_{1}}{\partial y}(0,0) \\
\frac{\partial f_{2}}{\partial x}(0,0) & \frac{\partial f_{2}}{\partial y}(0,0)
\end{array}\right)=0
$$

e $\frac{\partial f_{1}}{\partial x}(0,0), \frac{\partial f_{1}}{\partial y}(0,0), \frac{\partial f_{2}}{\partial x}(0,0), \frac{\partial f_{2}}{\partial y}(0,0)$ não todos nulos. A menos de mudança de coordenadas, podemos supor que $\frac{\partial f_{1}}{\partial x}(0,0) \neq 0$.

Seja $H(x, y)=\left(f_{1}(x, y), y\right)$. Temos que

$$
\operatorname{det}\left(\begin{array}{cc}
\frac{\partial f_{1}}{\partial x}(0,0) & \frac{\partial f_{1}}{\partial y}(0,0) \\
0 & 1
\end{array}\right)=\frac{\partial f_{1}}{\partial x}(0,0) \neq 0
$$

Assim $H$ é um germe de difeomorfismo. Ainda

$$
F \circ H^{-1}(x, y)=(x, \bar{F}(x, y))
$$

onde $\bar{F}=f_{2} \circ H^{-1}$.

Seja $F \in m_{2} \cdot \varepsilon_{2,2}$ um germe de corank 1 . Temos que $F(x, y)=(x, \bar{F}(x, y))$ onde $\frac{\partial \bar{F}}{\partial y}(0,0)=0 . \operatorname{Logo} j^{1} F(0,0)=(x, a x)$. Seja $K(x, y)=(x, y-a x)$, obtemos

$$
K \circ j^{1} F(0,0)(x, y)=K(x, a x)=(x, a x-a x)=(x, 0)
$$


Portanto $j^{1} F(0,0)$ é $\mathcal{A}^{1}$-equivalente a $(x, y) \mapsto(x, 0)$.

Basta então analisarmos o caso em que o 1-jato de $F$ é $(x, 0)$. Denotaremos este caso por I.1). Esta notação será utilizada em todos os casos, onde o número romano indicará a ordem do jato a ser trabalhado.

Temos que $(x, 0)$ não é finitamente $\mathcal{A}$-determinado. Encontremos então os elementos em $J^{2}(2,2)$ cujo 1-jato é $(x, 0)$. Temos que

$$
m_{2}^{2} \cdot \varepsilon_{2,2} \subset m_{2}^{2}\{(1,0)\}+f^{*} m_{2}^{2}\left\{e_{1}, e_{2}\right\}+m_{2}^{3} \cdot \varepsilon_{2,2}+T
$$

onde $T=\mathbb{R}\left\{\left(0, y^{2}\right),(0, x y)\right\}$ e $f(x, y)=(x, 0)$. Logo segue da Transversal Completa para jatos (3.1.3) que os elementos em $J^{2}(2,2)$ cujo 1-jato é $(x, 0)$ são $\mathcal{A}^{2}$-equivalentes a $\left(x, a x y+b y^{2}\right)$. Observemos que:

II.1.) se $b \neq 0$. Seja $h(x, y)=\left(x, a x y+b y^{2}\right)$. Tomemos $H(x, y)=$ $\left(x, y-\frac{a}{2 b} x\right)$, e $K(x, y)=\left(x, \frac{y}{b}+\frac{a^{2}}{4 b^{2}} x^{2}\right)$, mudanças de coordenadas . Então $K \circ h \circ H(x, y)=\left(x, y^{2}\right)$. Seja $f(x, y)=\left(x, y^{2}\right)$, observemos que

1. $m_{2}^{1} \cdot \varepsilon_{2,2} \subset \varepsilon_{2}\{(1,0),(0,2 y)\}+f^{*} m_{2} \cdot \varepsilon_{2}\left\{e_{1}, e_{2}\right\}$

2. $m_{2}^{1} \cdot \varepsilon_{2,2} \subset \varepsilon_{2}\{(1,0),(0,2 y)\}+f^{*} \varepsilon_{2}\left\{e_{1}, e_{2}\right\}$

assim, pelo Teorema 3.1.5, $f$ é 2 - $\mathcal{A}$-determinado. Portanto $F$ é $\mathcal{A}$-equivalente a $\left(x, y^{2}\right)$.

II.2.) se $b=0$ e $a \neq 0$. Sejam $h(x, y)=(x, a x y)$ e $K(x, y)=\left(x, \frac{1}{a} y\right)$. Temos que $K \circ h(x, y)=(x, x y)$. Se $f(x, y)=(x, x y)$, temos que

$$
T \mathcal{A} \cdot f=m_{2}\{(1, y),(0, x)\}+f^{*} m_{2}\left\{e_{1}, e_{2}\right\}
$$

$\operatorname{assim}\left(y^{k}, 0\right) \notin T \mathcal{A} \cdot f, \forall k \geq 1$. Logo $f$ tem $\mathcal{A}$-codimensão infinita.

II.3.) se $b=0$ e $a=0$. Neste caso, $j^{2} F(0,0)$ é $\mathcal{A}^{2}$-equivalente a $(x, 0)$ e tem $\mathcal{A}$-codimensão infinita. Aplicaremos a Transversal Completa (3.1.3) para os casos II.2) e II.3.).

- caso II.2.). Os elementos em $J^{3}(2,2)$, cujo 2-jato é $(x, x y)$, são $\mathcal{A}^{3}$-equivalentes a $\left(x, x y+a y^{3}\right)$ para algum $a \in \mathbb{R}$. 
III.1.) se $a \neq 0$. Seja $h(x, y)=\left(x, x y+a y^{3}\right)$. Tomando $H(x, y)=$ $\left(a^{\frac{1}{3}} x, \frac{1}{a^{\frac{1}{3}}} y\right)$ e $K(x, y)=\left(\frac{1}{a^{\frac{1}{3}}} x, y\right)$. Temos $K \circ h \circ H(x, y)=\left(x, x y+y^{3}\right)$. Seja $f(x, y)=\left(x, x y+y^{3}\right)$ e observemos que

1. $m_{2}^{2} \cdot \varepsilon_{2,2} \subset \varepsilon_{2}\left\{(1, y),\left(0, x+3 y^{2}\right)\right\}+f^{*} m_{2} \varepsilon_{2}\left\{e_{1}, e_{2}\right\}$

2. $m_{2}^{1} \cdot \varepsilon_{2,2} \subset \varepsilon_{2}\left\{(1, y),\left(0, x+3 y^{2}\right)\right\}+f^{*} \varepsilon_{2}\left\{e_{1}, e_{2}\right\}$

Pelo Teorema 3.1.5, temos que $f$ é 3-A-determinado, além disso tem $\mathcal{A}$ codimensão 2. Assim $F$ é $\mathcal{A}$-equivalente a $\left(x, x y+y^{3}\right)$.

III.2.) se $a=0$. Temos que $F$ é $\mathcal{A}^{3}$-equivalente a $(x, x y)$, mas $(x, x y)$ tem $\mathcal{A}$-codimensão infinita.

- caso II.3.). Os elementos em $J^{3}(2,2)$, cujo 2-jato é $(x, 0)$, são $\mathcal{A}^{3}$-equivalentes a $f(x, y)=\left(x, a x^{2} y+b x y^{2}+c y^{3}\right\}$, para alguns $a, b, c \in \mathbb{R}$.

Supondo que $c \neq 0$ e considerando

$$
\begin{gathered}
H(x, y)=\left(x, \bar{\alpha} x+\frac{y}{c^{\frac{1}{3}}}\right) \operatorname{com} \bar{\alpha}=\frac{-b}{3 c} \mathrm{e} \\
K(x, y)=\left(x, y-\alpha x^{3}\right) \operatorname{com} \alpha=\bar{\alpha} a+\bar{\alpha}^{2} b+\bar{\alpha}^{3} c
\end{gathered}
$$

obtemos $K \circ f \circ H(x, y)=\left(x, \beta x^{2} y+y^{3}\right)$ onde $\beta=\frac{a+2 \bar{\alpha} b+3 \bar{\alpha}^{2} c}{c^{\frac{1}{3}}}$. Assim

III.3.) se $\beta \neq 0$. Seja $f(x, y)=\left(x, \beta x y^{2}+y^{3}\right)$. Após mudanças de coordenadas obtemos que $f$ é $\mathcal{A}$-equivalente a

$$
g(x, y)=\left(x, x^{2} y \pm y^{3}\right)
$$

Temos que os elementos em $J^{4}(2,2)$, cujo 3 -jato é $\left(x, x^{2} y \pm y^{3}\right)$, são $\mathcal{A}^{4}$ equivalentes a $\left(x, x^{2} y \pm y^{3}\right)$. Observemos que

1. $m_{2}^{2} \cdot \varepsilon_{2,2} \subset \varepsilon_{2}\left\{(1,2 x y),\left(0, x^{2} \pm 3 y^{2}\right)\right\}+g^{*} m_{2} \varepsilon_{2}\left\{e_{1}, e_{2}\right\}$

2. $m_{2}^{2} \cdot \varepsilon_{2,2} \subset \varepsilon_{2}\left\{(1,2 x y),\left(0, x^{2} \pm 3 y^{2}\right)\right\}+g^{*} \varepsilon_{2}\left\{e_{1}, e_{2}\right\}$ 
Usando o Teorema 3.1.5, obtemos que $g$ é 4- $\mathcal{A}$-determinado, além disso $g$ tem $\mathcal{A}$-codimensão 3 . Portanto $F$ é $\mathcal{A}$-equivalente a $\left(x, x^{2} y \pm y^{3}\right)$.

III.4.) $\beta=0$. Seja $f(x, y)=\left(x, y^{3}\right)$, observemos que $\left(0, x^{k} y\right) \notin T \mathcal{A} \cdot f$, $\forall k \geq 1$, conseqüentemente $f$ tem $\mathcal{A}$-codimensão infinita.

III.5.) Vejamos o caso em que $c=0$ e $b \neq 0$, ou seja, consideramos $f(x, y)=\left(x, a x^{2} y+b x y^{2}\right)$.

Tomemos $H(x, y)=\left(\frac{x}{b}, y-\frac{a}{2 b^{2}} x\right)$ e $K(x, y)=\left(b x, y+\frac{a^{2}}{4 b} x^{3}\right)$, assim

$$
K \circ f \circ H(x, y)=\left(x, x y^{2}\right) .
$$

Seja $g(x, y)=\left(x, x y^{2}\right)$. Observemos que $\left(0, y^{k}\right) \notin T \mathcal{A} \cdot g, \forall k \geq 1$, assim $g$ tem $\mathcal{A}$-codimensão infinita.

III.6.) E no caso em que $b=c=0$ e $a \neq 0$, temos que $f(x, y)=\left(x, a x^{2} y\right)$. Tomemos $K(x, y)=\left(x, \frac{1}{a} y\right)$, assim

$$
K \circ f(x, y)=\left(x, x^{2} y\right) .
$$

Seja $g(x, y)=\left(x, x^{2} y\right)$. Temos que $g$ tem $\mathcal{A}$-codimensão infinita pois $\left(0, y^{k}\right) \notin T \mathcal{A} \cdot g, \forall k \geq 1$.

Aplicando a Transversal Completa (3.1.3) a $g(x, y)=\left(x, x^{2} y\right)$ em $J^{4}(2,2)$, obtemos que $j^{4} F(0,0)$ é $\mathcal{A}^{4}$-equivalente a

$$
h(x, y)=\left(x, x^{2} y+\alpha x^{3} y+\beta x^{2} y^{2}+\gamma x y^{3}+\delta y^{4}\right)
$$

Usando o software "Transversal" temos que $(0, y),\left(0, y^{2}\right),(0, x y),\left(0, y^{3}\right)$, $\left(0, x y^{2}\right) \notin T \mathcal{A} \cdot h$, independentemente de $\alpha, \beta, \gamma$ e $\delta$. Como eles são linearmente independentes, temos que os germes em $m_{2} \cdot \varepsilon_{2,2}$ cujo 3 -jato é $\left(x, x y^{2}\right)$ terão sempre $\mathcal{A}$-codimensão maior ou igual a 5 .

III.7.) Agora vejamos o caso em que $a=b=c=0$. Assim $f(x, y)=(x, 0)$, que tem $\mathcal{A}$-codimensão infinita. Ao aplicarmos a Transversal Completa (3.1.3) a $f$ em $J^{4}(2,2)$, obtemos que $j^{4} F(0,0)$ é $\mathcal{A}^{4}$-equivalente a

$$
h(x, y)=\left(x, a x^{4}+b x^{3} y+c x^{2} y^{2}+d x y^{3}+e y^{4}\right)
$$


Usando o software "Transversal" temos que $(0, y),\left(0, y^{2}\right),(0, x y),\left(0, y^{3}\right)$, $\left(0, x y^{2}\right) \notin T \mathcal{A} \cdot h$, independentemente dos valores de $a, b, c, d$ e $e$. Como são linearmente independentes, temos que os germes em $m_{2} \cdot \varepsilon_{2,2}$, cujo 3 -jato é $(x, 0)$, terão sempre $\mathcal{A}$-codimensão maior ou igual a 5 .

No que segue aplicaremos o método da Transversal Completa (3.1.3) aos casos III.2.), III.4) e III.5.). Consideremos em $J^{4}(2,2)$.

- Caso III.2.). Os elementos em $J^{4}(2,2)$ cujo 3-jato é $(x, x y)$ são $\mathcal{A}^{4}$-equivalentes a $\left(x, x y+a y^{4}\right)$. Assim

IV.1.) se $a \neq 0$. Seja $f(x, y)=\left(x, x y+a y^{4}\right)$. Consideremos

$$
H(x, y)=(a x, y) \text { e } K(x, y)=\left(\frac{x}{a}, \frac{y}{a}\right)
$$

Assim

$$
K \circ f \circ H(x, y)=\left(x, x y+y^{4}\right) .
$$

Seja $h(x, y)=\left(x, x y+y^{4}\right)$. Aplicando a Transversal Completa (3.1.3) a $h$ em $J^{5}(2,2)$, obtemos que os elementos, cujo 4 -jato é $h$, são $\mathcal{A}^{5}$-equivalente a $\left(x, x y+y^{4}+a y^{5}\right)$.

Seja $N=\left\{\left(x, x y+y^{4}+a y^{5}\right): a \in \mathbb{R}\right\} \subset J^{5}(2,2)$, temos que $N$ é uma variedade conexa e $T_{p} N=\mathbb{R}\left\{\left(0, y^{5}\right)\right\}, \forall p \in N$. Seja $G=\mathcal{A}^{5}$, temos que $T_{p} G \cdot p=\frac{T \mathcal{A} \cdot p+m_{2}^{6} \cdot \varepsilon_{2,2}}{m_{2}^{6} \cdot \varepsilon_{2,2}}$. E mais:

1. $T_{p} N \subset T_{p} G \cdot p, \forall p \in N$

2. $\operatorname{dim} T_{p} G \cdot p=37$, pois a $\mathcal{A}$-codimensão de $\left(x, x y+y^{4}+a y^{5}\right)$ é 3 , independente do valor de $a$.

Usando o Lema de Mather obtemos que $\left(x, x y+y^{4}+a y^{5}\right)$ é $\mathcal{A}^{5}$-equivalente a $\left(x, x y+y^{4}\right)$.

Aplicando novamente a Transversal Completa (3.1.3) a $h$ em $J^{6}(2,2)$, obtemos que os elementos, cujo 5 -jato é $h$, são $\mathcal{A}^{6}$-equivalentes a $(x, x y+$ $\left.y^{4}+b y^{6}\right)$. E seguindo o mesmo processo que em $J^{5}(2,2)$, obteremos pelo Lema de Mather que $\left(x, x y+y^{4}+b y^{6}\right)$ é $\mathcal{A}^{6}$-equivalente a $\left(x, x y+y^{4}\right)$.

Observemos que 
1. $m_{2}^{3} \cdot \varepsilon_{2,2} \subset \varepsilon_{2}\left\{(1, y),\left(0, x+4 y^{3}\right)\right\}+f^{*} m_{2} \cdot \varepsilon_{2}\left\{e_{1}, e_{2}\right\}$

2. $m_{2}^{3} \cdot \varepsilon_{2,2} \subset \varepsilon_{2}\left\{(1, y),\left(0, x+4 y^{3}\right)\right\}+f^{*} \varepsilon_{2}\left\{e_{1}, e_{2}\right\}$

Assim $\left(x, x y+y^{4}\right)$ é 6 - $\mathcal{A}$-determinado, pelo Teorema 3.1.5. Portanto $F$ é $\mathcal{A}$-equivalente a $\left(x, x y+y^{4}\right)$.

IV.2.) se $a=0$, temos que $j^{4} F(0,0)$ é $(x, x y)$, e como já vimos, tem $\mathcal{A}$ codimensão infinita.

- Consideremos o caso III.4.). Aplicando a Transversal Completa (3.1.3) obtemos que os elementos em $J^{4}(2,2)$, cujo 3-jato é $\left(x, y^{3}\right)$, são $\mathcal{A}^{4}$-equivalentes a $\left(x, y^{3}+a x^{3} y\right)$.

IV.3.) se $a \neq 0$. Seja $f(x, y)=\left(x, y^{3}+a x^{3} y\right)$. Tomando $H(x, y)=\left(a^{-\frac{1}{3}} x, y\right)$ e $K(x, y)=\left(a^{\frac{1}{3}} x, y\right)$, temos

$$
K \circ f \circ H(x, y)=\left(x, y^{3}+x^{3} y\right)
$$

Aplicando a Transversal Completa (3.1.3) a $K \circ f \circ H$ em $J^{5}(2,2)$, obtemos que os elementos, cujo 4 -jato é $\left(x, y^{3}+x^{3} y\right)$, são $\mathcal{A}^{5}$-equivalentes a $\left(x, y^{3}+x^{3} y\right)$.

Seja $g(x, y)=\left(x, y^{3}+x^{3} y\right)$. Observemos que

1. $m_{2}^{2} \cdot \varepsilon_{2,2} \subset \varepsilon_{2}\left\{\left(1,3 x^{2} y\right),\left(0,3 y^{2}+x^{3}\right)\right\}+g^{*} m_{2} \cdot \varepsilon_{2}\left\{e_{1}, e_{2}\right\}$

2. $m_{2}^{3} \cdot \varepsilon_{2,2} \subset \varepsilon_{2}\left\{\left(1,3 x^{2} y\right),\left(0,3 y^{2}+x^{3}\right)\right\}+g^{*} \varepsilon_{2}\left\{e_{1}, e_{2}\right\}$

Assim $g$ é 5 - $\mathcal{A}$-determinado, pelo Teorema 3.1.5. Portanto $F$ é $\mathcal{A}$-equivalente a $g$.

IV.4.) se $a=0$. Seja $f(x, y)=\left(x, y^{3}\right)$. Quando aplicamos a Transversal Completa (3.1.3) a $f$ em $J^{5}(2,2)$, temos que os elementos, cujo 4-jato é $f$, são $\mathcal{A}^{5}$-equivalentes a

$$
h(x, y)=\left(x, y^{3}+a x^{4} y\right)
$$

Usando o software "Transversal" temos que $(0, y),\left(0, y^{2}\right),(0, x y),\left(0, x^{2} y\right)$, $\left(0, x^{3} y\right) \notin T \mathcal{A} \cdot h$, independentemente de $a$. Como são linearmente independentes, temos que os germes em $m_{2} \cdot \varepsilon_{2,2}$, cujo 4-jato é $\left(x, y^{3}\right)$, terão sempre 
codimensão maior ou igual à 5 .

- Consideremos agora o caso III.5.). Aplicando a Transversal Completa (3.1.3) temos que os elementos, cujo 3 -jato é $\left(x, x y^{2}\right)$, são $\mathcal{A}^{4}$-equivalentes a $\left(x, x y^{2}+a y^{4}\right)$.

IV.5.) se $a \neq 0$. Seja $f(x, y)=\left(x, x y^{2}+a y^{4}\right)$, tomemos

$$
H(x, y)=(a x, y) \text { e } K(x, y)=\left(\frac{x}{a}, \frac{y}{a}\right)
$$

$\log \mathrm{O}$

$$
K \circ f \circ H(x, y)=\left(x, x y^{2}+y^{4}\right)
$$

Daremos continuidade a este caso mais adiante.

IV.6.) se $a=0$. Seja $f(x, y)=\left(x, x y^{2}\right)$. Aplicando a Transversal Completa (3.1.3) a $f$ em $J^{5}(2,2)$, obtemos que os elementos cujo 4-jato é $\left(x, x y^{2}\right)$ são $\mathcal{A}^{5}$-equivalentes a

$$
h(x, y)=\left(x, x y^{2}+a y^{5}\right)
$$

Mas usando o software "Transversal" temos que $(0, y),\left(0, y^{2}\right),(0, x y)$, $\left(0, y^{3}\right),\left(0, y^{4}\right) \notin T \mathcal{A} \cdot h$, independentemente de $a$. Como são linearmente independentes, temos que os germes em $m_{2} \cdot \varepsilon_{2,2}$ cujo 4-jato é $\left(x, x y^{2}\right)$ terão sempre $\mathcal{A}$-codimensão maior ou igual à 5 .

Aplicaremos a Transversal Completa (3.1.3) aos casos IV.2.) e IV.5.).

- No caso IV.2.). Os elementos em $J^{5}(2,2)$, cujo 4-jato é $(x, x y)$, são $\mathcal{A}^{5}$ equivalentes a $\left(x, x y+a y^{5}\right)$.

V.1.) se $a \neq 0$. Seja $f(x, y)=\left(x, x y+a y^{5}\right)$. Tomando $H(x, y)=(a x, y)$ e $K(x, y)=\left(\frac{x}{a}, \frac{y}{a}\right)$ obtemos

$$
K \circ f \circ H(x, y)=\left(x, x y+y^{5}\right)
$$

Daremos continuidade a este caso mais adiante.

V.2.) se $a=0$. Seja $f(x, y)=(x, x y)$. Observemos que ao aplicarmos 
a Transversal Completa (3.1.3) obtemos que os elementos em $J^{6}(2,2)$, cujo 5 -jato é $(x, x y)$, são $\mathcal{A}^{6}$-equivalentes a

$$
h(x, y)=\left(x, x y+a y^{6}\right)
$$

mas $(0, y),\left(0, y^{2}\right),\left(0, y^{3}\right),\left(0, y^{4}\right),\left(0, y^{5}\right) \notin T \mathcal{A} \cdot h$, independentemente de $a$. Como são linearmente independentes, temos que os germes em $m_{2} \cdot \varepsilon_{2,2}$, cujo 5 -jato é $(x, x y)$, terão sempre $\mathcal{A}$-codimensão maior ou igual a 5 .

- Consideremos agora o caso IV.5.). Os elementos em $J^{5}(2,2)$, cujo 4-jato é $\left(x, x y^{2}+y^{4}\right)$, são $\mathcal{A}^{5}$-equivalentes a $\left(x, x y^{2}+y^{4}+a y^{5}\right)$.

V.3.) se $a \neq 0$. Após uma mudança de coordenadas podemos considerar $f(x, y)=\left(x, x y^{2}+y^{4}+y^{5}\right)$.

A transversal completa de $f$ em $J^{k}(2,2), k \geq 6$, é vazia. Além disso

$$
T \mathcal{A} \cdot g \subset \varepsilon_{2}\left\{\left(1, y^{2}\right),\left(0,2 x y+4 y^{3}+5 y^{4}\right)\right\}+g^{*} m_{2} \cdot \varepsilon_{2}\left\{e_{1}, e_{2}\right\}
$$

e

$$
T \mathcal{A} \cdot g \subset \varepsilon_{2}\left\{\left(1, y^{2}\right),\left(0,2 x y+4 y^{3}+5 y^{4}\right)\right\}+g^{*} \varepsilon_{2}\left\{e_{1}, e_{2}\right\}
$$

Assim

$$
\frac{m_{2} \cdot \varepsilon_{2,2}}{T \mathcal{A} \cdot g} \supset \frac{m_{2} \cdot \varepsilon_{2,2}}{\varepsilon_{2}\left\{\left(1, y^{2}\right),\left(0,2 x y+4 y^{3}+5 y^{4}\right)\right\}+g^{*} m_{2} \cdot \varepsilon_{2}\left\{e_{1}, e_{2}\right\}}
$$

e

$$
\frac{m_{2} \cdot \varepsilon_{2,2}}{T \mathcal{A} \cdot g} \supset \frac{m_{2} \cdot \varepsilon_{2,2}}{\varepsilon_{2}\left\{\left(1, y^{2}\right),\left(0,2 x y+4 y^{3}+5 y^{4}\right)\right\}+g^{*} \varepsilon_{2}\left\{e_{1}, e_{2}\right\}}
$$

Usando o software "Transversal" temos

$$
\frac{m_{2} \cdot \varepsilon_{2,2}}{T \mathcal{A} \cdot g}=\mathbb{R}\left\{(0, y),\left(0, y^{2}\right),(0, x y),\left(0, y^{3}\right)\right\}
$$

Assim

$$
m_{2}^{4} \cdot \varepsilon_{2,2} \subset \varepsilon_{2}\left\{\left(1, y^{2}\right),\left(0,2 x y+4 y^{3}+5 y^{4}\right)\right\}+g^{*} m_{2} \cdot \varepsilon_{2}\left\{e_{1}, e_{2}\right\}
$$

e

$$
m_{2}^{4} \cdot \varepsilon_{2,2} \subset \varepsilon_{2}\left\{\left(1, y^{2}\right),\left(0,2 x y+4 y^{3}+5 y^{4}\right)\right\}+g^{*} \varepsilon_{2}\left\{e_{1}, e_{2}\right\}
$$

Logo pelo Teorema 3.1.5 temos que $g$ é 8- $\mathcal{A}$-determinado. Assim, se $j^{5} F(0,0)$ $=\left(x, x y^{2}+y^{4}+y^{5}\right)$ temos que $F$ é $\mathcal{A}$-equivalente a $g$. 
V.4.) se $a=0$. Seja $f(x, y)=\left(x, x y^{2}+y^{4}\right)$. A transversal completa de $f$ em $J^{6}(2,2)$ é vazia.

Mas, aplicando a Transversal Completa (3.1.3) a $f$ em $J^{7}(2,2)$ para encontrar os elementos, cujo 6-jato é $\left(x, x y^{2}+y^{4}\right)$, são $\mathcal{A}^{7}$-equivalentes a $h(x, y)=\left(x, x y^{2}+y^{4}+a y^{7}\right), \operatorname{mas}(0, y),\left(0, y^{2}\right),(0, x y),\left(0, y^{3}\right),\left(0, y^{5}\right) \notin T \mathcal{A} \cdot h$, independentemente de $a$. Como são linearmente independentes, temos que os germes em $m_{2} \cdot \varepsilon_{2,2}$, cujo 6 -jato é $\left(x, x y^{2}+y^{4}\right)$, terão sempre $\mathcal{A}$-codimensão maior ou igual a 5 .

Consideremos o caso V.1.). Os elementos, cujo 5-jato é $\left(x, x y+y^{5}\right)$, são $\mathcal{A}$-equivalentes a $\left(x, x y+y^{5}+a y^{6}\right)$.

Seja $N=\left\{\left(x, x y+y^{5}+a y^{6}\right): a \in \mathbb{R}\right\} \subset J^{5}(2,2)$, temos que $T_{p} N=$ $\mathbb{R}\left\{\left(0, y^{6}\right)\right\}, \forall p \in N$. Seja $G=\mathcal{A}^{6}$, temos

$$
T_{p} G \cdot p=\frac{m_{2}\left\{\frac{\partial p}{\partial x}, \frac{\partial p}{\partial y}\right\}+p^{*} m_{2}\left\{e_{1}, e_{2}\right\}+m_{2}^{7} \cdot \varepsilon_{2,2}}{m_{2}^{7} \cdot \varepsilon_{2,2}}
$$

e $\operatorname{dim} T_{p} G \cdot p=36$. Pelo Lema de Mather (1.2.6) obtemos que $\left(x, x y+y^{5}+a y^{6}\right)$ é $\mathcal{A}^{6}$-equivalente a $\left(x, x y+y^{5}\right)$. Denotemos este caso por VI.1.).

Ao aplicarmos a Transversal Completa (3.1.3) temos que os elementos em $J^{7}(2,2)$, cujo 6 -jato é $\left(x, x y+y^{5}\right)$, são $\mathcal{A}^{7}$-equivalentes a $\left(x, x y+y^{5}+a y^{7}\right)$.

VII.1.) se $a \neq 0$. Após uma mudança de coordenadas podemos supor que $f(x, y)=\left(x, x y+y^{5} \pm y^{7}\right)$.

Seja $g(x, y)=\left(x, x y+y^{5}+y^{7}\right)$. A transversal completa a $g$ em $J^{k}(2,2), k \geq$ 8 , é vazia. Ainda

$$
T \mathcal{A} \cdot g \subset \varepsilon_{2}\left\{(1, y),\left(0, x+5 y^{4}+7 y^{6}\right)\right\}+g^{*} m_{2} \cdot \varepsilon_{2}\left\{e_{1}, e_{2}\right\}
$$

e

$$
T \mathcal{A} \cdot g \subset \varepsilon_{2}\left\{(1, y),\left(0, x+5 y^{4}+7 y^{6}\right)\right\}+g^{*} \varepsilon_{2}\left\{e_{1}, e_{2}\right\}
$$

Assim

$$
\frac{m_{2} \cdot \varepsilon_{2,2}}{T \mathcal{A} \cdot g} \supset \frac{m_{2} \cdot \varepsilon_{2,2}}{\varepsilon_{2}\left\{(1, y),\left(0, x+5 y^{4}+7 y^{6}\right)\right\}+g^{*} m_{2} \cdot \varepsilon_{2}\left\{e_{1}, e_{2}\right\}}
$$


e

$$
\frac{m_{2} \cdot \varepsilon_{2,2}}{T \mathcal{A} \cdot g} \supset \frac{m_{2} \cdot \varepsilon_{2,2}}{\varepsilon_{2}\left\{(1, y),\left(0, x+5 y^{4}+7 y^{6}\right)\right\}+g^{*} \varepsilon_{2}\left\{e_{1}, e_{2}\right\}}
$$

Usando o software "Transversal" obtemos

$$
\frac{m_{2} \cdot \varepsilon_{2,2}}{T \mathcal{A} \cdot g}=\mathbb{R}\left\{(0, y),\left(0, y^{2}\right),\left(0, y^{3}\right),\left(0, y^{4}\right)\right\}
$$

Assim

$$
m_{2}^{5} \cdot \varepsilon_{2,2} \subset \varepsilon_{2}\left\{(1, y),\left(0, x+5 y^{4}+7 y^{6}\right)\right\}+g^{*} m_{2} \cdot \varepsilon_{2}\left\{e_{1}, e_{2}\right\}
$$

$\mathrm{e}$

$$
m_{2}^{5} \cdot \varepsilon_{2,2} \subset \varepsilon_{2}\left\{(1, y),\left(0, x+5 y^{4}+7 y^{6}\right)\right\}+g^{*} \varepsilon_{2}\left\{e_{1}, e_{2}\right\}
$$

Logo pelo Teorema 3.1.5 temos que $g$ é $10-\mathcal{A}$-determinado. Portanto, se $j^{7} F(0,0)=\left(x, x y+y^{5}+y^{7}\right)$ então $F$ é $\mathcal{A}$-equivalente a $g$. Da mesma forma obtemos que se $j^{7} F(0,0)=\left(x, x y+y^{5}-y^{7}\right)$ então $F$ é $\mathcal{A}$-equivalente a $\left(x, x y+y^{5}-y^{7}\right)$.

VII.2.) se $a=0$. Seja $f(x, y)=\left(x, x y+y^{5}\right)$. Os elementos em $J^{8}(2,2)$, cujo 7 -jato é $f$, são $\mathcal{A}^{8}$-equivalentes a $h(x, y)=\left(x, x y+y^{5}+a y^{8}\right)$.

Mas $(0, y),\left(0, y^{2}\right),\left(0, y^{3}\right),\left(0, y^{4}\right),\left(0, y^{7}\right) \notin T \mathcal{A} \cdot h$, independentemente de $a$, e além disso são linearmente independentes. Com isto temos que qualquer elemento em $J^{k}(2,2), k \geq 8$, cujo 7 -jato é $\left(x, x y+y^{5}\right)$ terá $\mathcal{A}$-codimensão maior ou igual a 5 .

O seguinte resultado é a conclusão desta seção:

Teorema 3.2.2 Seja $f \in m_{2} \cdot \varepsilon_{2,2}$ um germe de corank 1 e $\mathcal{A}$-codimensão $\leq 4$. Então f é $\mathcal{A}$-equivalente a um dos seguintes germes:

$$
\begin{array}{llll}
(x, y) & \mapsto\left(x, y^{2}\right) & , \mathcal{A}-\operatorname{cod}=1 ; \\
(x, y) & \mapsto\left(x, x y+y^{3}\right) & , \mathcal{A}-\operatorname{cod}=2 ; \\
(x, y) \mapsto\left(x, x^{2} y \pm y^{3}\right) & , \mathcal{A}-\operatorname{cod}=3 ; \\
(x, y) \mapsto\left(x, x y+y^{4}\right) & , \mathcal{A}-\operatorname{cod}=3 ; \\
(x, y) \mapsto\left(x, x^{3} y+y^{3}\right) & , \mathcal{A}-\operatorname{cod}=4 ; \\
(x, y) \mapsto\left(x, x y^{2}+y^{4}+y^{5}\right) & , \mathcal{A}-\operatorname{cod}=4 ; \\
(x, y) \mapsto\left(x, x y+y^{5} \pm y^{7}\right) & , \mathcal{A}-\operatorname{cod}=4
\end{array}
$$




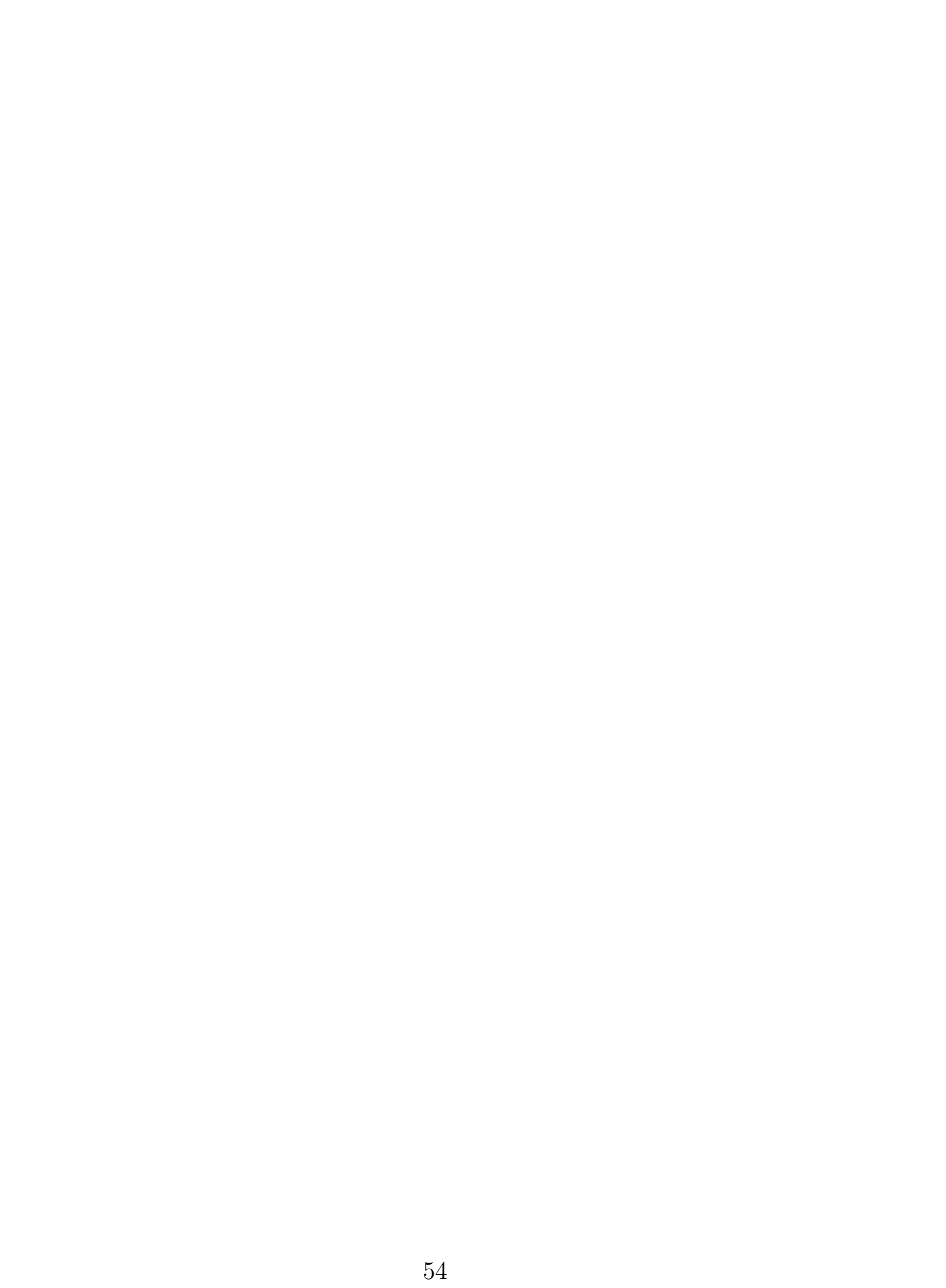




\section{Capítulo 4}

\section{Classificação de Bigermes de $(\mathbb{R}, S) \rightarrow\left(\mathbb{R}^{2}, 0\right)$}

O objetivo deste capítulo é apresentar a classificação dos bigermes cujos ramos têm corank 0 e uma classificação parcial dos bigermes cujo um ramo tem corank 0 e o outro corank 1, para isto usaremos o Método da Transversal Completa apresentada na seção 2.2 .

\subsection{Resultados Gerais}

Seja $S=\left\{p_{1}, p_{2}\right\}$ um subconjunto de $\mathbb{R}$. No conjunto das aplicações de classe $C^{\infty}$ definidas numa vizinhança de $S \subset \mathbb{R}$ com valores em $\mathbb{R}^{2}$, introduzimos a seguinte relação de equivalência: duas aplicações $f: U \rightarrow \mathbb{R}^{2}$ e $g: V \rightarrow \mathbb{R}^{2}$ são equivalentes se existir uma vizinhança $W$ de $S$ em $\mathbb{R}$ tal que $\left.f\right|_{W}=\left.g\right|_{W}$. A classe de equivalência é chamada bigerme de $f$ em $S$ e denotados por $f:(\mathbb{R}, S) \rightarrow\left(\mathbb{R}^{2}, f(S)\right)$.

Suponhamos $f(S)=0$, o germe de $f$ em $p_{i}$ é chamado de ramo e denotado por $f_{i}:\left(\mathbb{R}, p_{i}\right) \rightarrow\left(\mathbb{R}^{2}, 0\right), i=1,2$. Assim denotamos $f$ por

$$
f:\left\{\begin{array}{l}
x \mapsto f_{1}(x) \\
\bar{x} \mapsto f_{2}(\bar{x})
\end{array}\right.
$$

onde $f_{1}(x)=\left(f_{11}(x), f_{12}(x)\right)$ e $f_{2}(\bar{x})=\left(f_{21}(\bar{x}), f_{22}(\bar{x})\right)$ 
Dois bigermes $f, g:(\mathbb{R}, S) \rightarrow\left(\mathbb{R}^{2}, 0\right)$ são ditos $\mathcal{A}$-equivalentes se existirem germes de difeomorfismos $\varphi:(\mathbb{R}, S) \rightarrow \mathbb{R}, \varphi(S)=S$ e $\psi:\left(\mathbb{R}^{2}, 0\right) \rightarrow\left(\mathbb{R}^{2}, 0\right)$ tais que $f \circ \varphi=\psi \circ g$.

O conjunto dos bigermes $f:(\mathbb{R}, S) \rightarrow\left(\mathbb{R}^{2}, 0\right)$ é um $\varepsilon_{1}$-módulo isomorfo a $m_{1} \cdot \varepsilon_{1,2} \oplus m_{1} \cdot \varepsilon_{1,2}$.

Definição 4.1.1 O espaço tangente ao bigerme $f:(\mathbb{R}, S) \rightarrow\left(\mathbb{R}^{2}, 0\right)$ segundo o grupo $\mathcal{A}$ é dado por

$$
\begin{gathered}
T \mathcal{A} \cdot f=\left\{\begin{array}{cc}
\left(\begin{array}{ll}
\xi \frac{d f_{11}}{d x}(0) & \zeta \frac{d f_{21}}{d \bar{x}}(0) \\
\xi \frac{d f_{12}}{d x}(0) & \zeta \frac{d f_{22}}{d \bar{x}}(0)
\end{array}\right)+\left(\begin{array}{cc}
\eta_{1} \circ f_{1}(x) & \eta_{1} \circ f_{2}(x) \\
\eta_{2} \circ f_{1}(x) & \eta_{2} \circ f_{2}(x)
\end{array}\right), \\
\xi, \zeta \in m_{1} \text { ○ } \eta_{1}, \eta_{2} \in m_{2}
\end{array}\right\}
\end{gathered}
$$

E o espaço tangente estendido ao bigerme $f:(\mathbb{R}, 0) \rightarrow\left(\mathbb{R}^{2}, 0\right)$ segundo o grupo $\mathcal{A}$ é dado por

$$
\left.\begin{array}{rl}
T \mathcal{A}_{e} \cdot f= & \left\{\begin{array}{cc}
\xi \frac{d f_{11}}{d x}(0) & \zeta \frac{d f_{21}}{d \bar{x}}(0) \\
\xi \frac{d f_{12}}{d x}(0) & \zeta \frac{d f_{22}}{d \bar{x}}(0)
\end{array}\right)+\left(\begin{array}{cc}
\eta_{1} \circ f_{1}(x) & \eta_{1} \circ f_{2}(x) \\
\eta_{2} \circ f_{1}(x) & \eta_{2} \circ f_{2}(x)
\end{array}\right), \\
\xi, \zeta \in \varepsilon_{1} & \text { e } \eta_{1}, \eta_{2} \in \varepsilon_{2}
\end{array}\right\}
$$

Definição 4.1.2 Seja $f:(\mathbb{R}, S) \rightarrow\left(\mathbb{R}^{2}, 0\right)$. A $\mathcal{A}$-codimensão de $f$ é definida por

$$
\mathcal{A}-\operatorname{cod} f=\operatorname{dim} \frac{m_{1} \cdot \varepsilon_{1,2} \oplus m_{1} \cdot \varepsilon_{1,2}}{T \mathcal{A} \cdot f}
$$

e a $\mathcal{A}_{e}$-codimensão de $f$ é definida por

$$
\mathcal{A}_{e}-\operatorname{cod} f=\operatorname{dim} \frac{m_{1} \cdot \varepsilon_{1,2} \oplus m_{1} \cdot \varepsilon_{1,2}}{T \mathcal{A}_{e} \cdot f}
$$

Teorema 4.1.3 Seja $f:(\mathbb{R}, S) \rightarrow\left(\mathbb{R}^{2}, 0\right)$. Se $\mathcal{A}_{e}-\operatorname{cod} f>0$ então temos a seguinte relação

$$
\mathcal{A}_{e}-\operatorname{cod} f=\mathcal{A}-\operatorname{cod} f
$$




\section{Demonstração:}

Ver [8].

Seja $f:(\mathbb{R}, S) \rightarrow\left(\mathbb{R}^{2}, 0\right)$, definimos o espaço tangente a $f$ segundo o grupo $\mathcal{A}_{1}$ por

$$
\left.\begin{array}{rl}
T \mathcal{A}_{1} \cdot f= & \left\{\begin{array}{cc}
\xi \frac{d f_{11}}{d x}(0) & \zeta \frac{d f_{21}}{d \bar{x}}(0) \\
\xi \frac{d f_{12}}{d x}(0) & \zeta \frac{d f_{22}}{d \bar{x}}(0)
\end{array}\right)+\left(\begin{array}{cc}
\eta_{1} \circ f_{1}(x) & \eta_{1} \circ f_{2}(x) \\
\eta_{2} \circ f_{1}(x) & \eta_{2} \circ f_{2}(x)
\end{array}\right), \\
\xi, \zeta \in m_{1}^{2} \text { e } \eta_{1}, \eta_{2} \in m_{2}^{2}
\end{array}\right\}
$$

O resultado seguinte é conseqüência da Proposição 2.2.1.

Proposição 4.1.4 (Transversal Completa para jatos de bigermes) Sejam $f \in J^{k}(1,2) \oplus J^{k}(1,2)$ e $T \subset H^{k+1}(1,2) \oplus H^{k+1}(1,2)$ subespaços tais que

$$
T \mathcal{A}_{1} \cdot f+T+m_{1}^{k+2} \cdot \varepsilon_{1,2} \oplus m_{1}^{k+2} \cdot \varepsilon_{1,2} \supset m_{1}^{k+1} \cdot \varepsilon_{1,2} \oplus m_{1}^{k+1} \cdot \varepsilon_{1,2}
$$

Então qualquer $g \in J^{k+1}(1,2) \oplus J^{k+1}(1,2) \operatorname{com} j^{k} g(0)=f$ é $\mathcal{A}_{1}^{k}$-equivalente a $f+\beta \operatorname{com} \beta \in T$.

\subsection{A Classificação}

Seja $f:(\mathbb{R}, S) \rightarrow\left(\mathbb{R}^{2}, 0\right)$ um bigerme dado por

$$
f:\left\{\begin{array}{l}
x \mapsto f_{1}(x) \\
\bar{x} \mapsto f_{2}(\bar{x})
\end{array}\right.
$$

onde $f_{1}(x)=\left(f_{11}(x), f_{12}(x)\right)$ e $f_{2}(\bar{x})=\left(f_{21}(\bar{x}), f_{22}(\bar{x})\right)$.

Nesta seção faremos a classificação dos bigermes cujos ramos têm corank zero e uma pequena classificação dos bigermes onde cada ramo tem corank 
0 ou 1.

- Suponhamos que os corank de $f_{1}$ e $f_{2}$ sejam zero. Como $f_{1}$ é imersão, segue da Forma Local das Imersões (1.1.4) que $f$ é $\mathcal{A}$-equivalente a

$$
g:\left\{\begin{array}{l}
x \stackrel{g_{1}}{\longmapsto}(x, 0) \\
\bar{x} \stackrel{g_{2}}{\longmapsto}\left(g_{21}(\bar{x}), g_{22}(\bar{x})\right)
\end{array}\right.
$$

Se $\frac{d g_{21}}{d \bar{x}}(0) \neq 0$. Seja $c=\frac{d g_{21}}{d \bar{x}}(0)$. Tomando $K(x, y)=\left(\frac{1}{c} x, y\right)$ e $H(x)=$ $c x$, temos que

$$
K \circ j^{1} g_{1}(0) \circ H(x)=(x, 0) \text { e } K \circ j^{1} g_{2}(0)(\bar{x})=\left(\bar{x}, j^{1} g_{22}(0)\right)
$$

assim $j^{1} g(0)$ é $\mathcal{A}$-equivalente a

$$
h:\left\{\begin{array}{l}
x \longmapsto(x, 0) \\
\bar{x} \longmapsto\left(\bar{x}, j^{1} g_{22}(0)\right)
\end{array}\right.
$$

Aqui temos dois subcasos:

1) se $\frac{d g_{22}}{d \bar{x}}(0) \neq 0$. Sejam $c_{0}=\frac{d g_{21}}{d \bar{x}}(0), c_{1}=\frac{d g_{22}}{d \bar{x}}(0)$ e tomando $K(x, y)=$ $\left(x-\frac{c_{0}}{c_{1}} y, \frac{1}{c_{1}} y\right)$, temos que

$$
K \circ h:\left\{\begin{array}{l}
x \longmapsto(x, 0) \\
\bar{x} \longmapsto(0, \bar{x})
\end{array}\right.
$$

denotemos $\widetilde{h}=K \circ h$, assim $h$ é $\mathcal{A}^{1}$-equivalente a $\widetilde{h}$. E mais, ao aplicarmos a Transversal Completa (4.1.4) temos que os elementos em $J^{2}(2,1) \oplus J^{2}(2,1)$, cujo 1 -jato é $\widetilde{h}$, são $\mathcal{A}^{2}$-equivalentes a $\widetilde{h}$. Sejam

$$
\begin{aligned}
T \mathcal{K}_{e} \cdot \widetilde{h}= & \left\{\left(\begin{array}{cc}
\xi & 0 \\
0 & \zeta
\end{array}\right)+\left(\begin{array}{cc}
\delta \eta_{1}(x, 0) & \gamma \eta_{1}(0, \bar{x}) \\
\delta \eta_{2}(x, 0) & \gamma \eta_{2}(0, \bar{x})
\end{array}\right),\right. \\
& \left.\xi, \zeta, \delta, \gamma \in \varepsilon_{1} \text { e } \eta_{1}, \eta_{2} \in m_{2}\right\}
\end{aligned}
$$

e

$$
T \mathcal{A}_{e} \cdot \widetilde{h}=\left\{\left(\begin{array}{cc}
\xi & 0 \\
0 & \zeta
\end{array}\right)+\left(\begin{array}{ll}
\eta_{1}(x, 0) & \eta_{1}(0, \bar{x}) \\
\eta_{2}(x, 0) & \eta_{2}(0, \bar{x})
\end{array}\right), \xi, \zeta \in \varepsilon_{1} \text { e } \eta_{1}, \eta_{2} \in \varepsilon_{2}\right\}
$$


Temos que $m_{1}^{1} \cdot \varepsilon_{1,2} \oplus m_{1}^{1} \cdot \varepsilon_{1,2} \subset T \mathcal{K}_{e} \cdot \widetilde{h}$ e $m_{1}^{1} \cdot \varepsilon_{1,2} \oplus m_{1}^{1} \cdot \varepsilon_{1,2} \subset T \mathcal{A}_{e} \cdot \widetilde{h}$, pelo Teorema 3.1.5 temos que $\widetilde{h}$ é 2 - $\mathcal{A}$-determinado. Portanto $f$ é $\mathcal{A}$-equivalente a $\widetilde{h}$.

2) se $\frac{d g_{22}}{d x}(0)=0$, assim

$$
j^{1} g(0):\left\{\begin{array}{l}
x \longmapsto(x, 0) \\
\bar{x} \longmapsto(\bar{x}, 0)
\end{array}\right.
$$

Temos que $j^{1} g(0)$ tem codimensão infinita, pois

$$
\begin{aligned}
\left(\begin{array}{cc}
0 & 0 \\
0 & \bar{x}^{k}
\end{array}\right) \notin T \mathcal{A} \cdot j^{1} g(0)= & \left\{\begin{array}{ll}
\xi & \zeta \\
0 & 0
\end{array}\right)+\left(\begin{array}{ll}
\eta_{1}(x, 0) & \eta_{1}(\bar{x}, 0) \\
\eta_{2}(x, 0) & \eta_{2}(\bar{x}, 0)
\end{array}\right), \\
& \left.\xi, \zeta \in m_{1} \text { e } \eta_{1}, \eta_{2} \in m_{2}\right\}
\end{aligned}
$$

com $k \geq 1$. Ao aplicarmos a Transversal Completa 4.1.4 temos que os elementos em $J^{2}(1,2) \oplus J^{2}(1,2)$, cujo 1 -jato é $j^{1} g(0)$, são $\mathcal{A}^{2}$-equivalentes a

$$
h:\left\{\begin{array}{l}
x \longmapsto(x, 0) \\
\bar{x} \longmapsto\left(\bar{x}, a \bar{x}^{2}\right)
\end{array}\right.
$$

Se $a \neq 0$, tomando $K_{1}(x, y)=\left(x, \frac{1}{a} y\right)$ temos que

$$
K_{1} \circ h:\left\{\begin{array}{l}
x \longmapsto(x, 0) \\
\bar{x} \longmapsto\left(\bar{x}, \bar{x}^{2}\right)
\end{array}\right.
$$

seja $\widetilde{h}=K_{1} \circ h$, assim $h$ é $\mathcal{A}$-equivalente a $\widetilde{h}$. E mais, aplicando a Transversal Completa (4.1.4) temos que os elementos em $J^{k}(1,2) \oplus J^{k}(1,2)$, cujo 2-jato é $\widetilde{h}$, são $\mathcal{A}^{k}$-equivalentes a $\widetilde{h}, k \geq 3$.

Sejam

$$
\begin{aligned}
T \mathcal{K}_{e} \cdot \widetilde{h}= & \left\{\left(\begin{array}{cc}
\xi & \zeta \\
0 & 2 \zeta \bar{x}
\end{array}\right)+\left(\begin{array}{cc}
\delta \eta_{1}(x, 0) & \gamma \eta_{1}\left(\bar{x}, \bar{x}^{2}\right) \\
\delta \eta_{2}(x, 0) & \gamma \eta_{2}\left(\bar{x}, \bar{x}^{2}\right)
\end{array}\right),\right. \\
& \left.\xi, \zeta, \delta, \gamma \in \varepsilon_{1} \text { e } \eta_{1}, \eta_{2} \in m_{2}\right\}
\end{aligned}
$$

e

$$
T \mathcal{A}_{e} \cdot \widetilde{h}=\left\{\left(\begin{array}{cc}
\xi & \zeta \\
0 & 2 \zeta \bar{x}
\end{array}\right)+\left(\begin{array}{cc}
\eta_{1}(x, 0) & \eta_{1}\left(\bar{x}, \bar{x}^{2}\right) \\
\eta_{2}(x, 0) & \eta_{2}\left(\bar{x}, \bar{x}^{2}\right)
\end{array}\right), \xi, \zeta \in \varepsilon_{1} \text { e } \eta_{1}, \eta_{2} \in \varepsilon_{2}\right\}
$$


Observemos que $T \mathcal{A} \cdot \widetilde{h} \subset T \mathcal{K}_{e} \cdot \widetilde{h}$ e $T \mathcal{A} \cdot \widetilde{h} \subset T \mathcal{A}_{e} \cdot \widetilde{h}$, daí

$$
\frac{m_{1} \cdot \varepsilon_{1,2} \oplus m_{1} \cdot \varepsilon_{1,2}}{T \mathcal{A} \cdot \widetilde{h}} \supset \frac{m_{1} \cdot \varepsilon_{1,2} \oplus m_{1} \cdot \varepsilon_{1,2}}{T \mathcal{K}_{e} \cdot \widetilde{h}}
$$

e

$$
\frac{m_{1} \cdot \varepsilon_{1,2} \oplus m_{1} \cdot \varepsilon_{1,2}}{T \mathcal{A} \cdot \widetilde{h}} \supset \frac{m_{1} \cdot \varepsilon_{1,2} \oplus m_{1} \cdot \varepsilon_{1,2}}{T \mathcal{A}_{e} \cdot \widetilde{h}}
$$

Mas

$$
\frac{m_{1} \cdot \varepsilon_{1,2} \oplus m_{1} \cdot \varepsilon_{1,2}}{T \mathcal{A} \cdot \widetilde{h}}=\mathbb{R}\left\{\left(\begin{array}{ll}
0 & 0 \\
x & 0
\end{array}\right)\right\}
$$

$\operatorname{assim} m_{1}^{2} \cdot \varepsilon_{1,2} \oplus m_{1}^{2} \cdot \varepsilon_{1,2} \subset T \mathcal{K}_{e} \cdot \widetilde{h}$ e $m_{1}^{2} \cdot \varepsilon_{1,2} \oplus m_{1}^{2} \cdot \varepsilon_{1,2} \subset T \mathcal{A}_{e} \cdot \widetilde{h}$, pelo Teorema 3.1.5 temos que $\widetilde{h}$ é 4 - $\mathcal{A}$-determinado. Portanto $f$ é $\mathcal{A}$-equivalente a $\widetilde{h}$.

Queremos encontrar os elementos em $J^{k+1}(1,2) \oplus J^{k+1}(1,2)$ cujo o $k$-jato é

$$
h:\left\{\begin{array}{l}
x \longmapsto(x, 0) \\
\bar{x} \longmapsto(\bar{x}, 0)
\end{array}\right.
$$

com $k \geq 2$. Ao aplicarmos a Transversal Completa (4.1.4), obtemos que tais elementos são $\mathcal{A}^{k+1}$-equivalentes a

$$
\widetilde{h}:\left\{\begin{array}{l}
x \longmapsto(x, 0) \\
\bar{x} \longmapsto\left(\bar{x}, b \bar{x}^{k+1}\right)
\end{array}\right.
$$

Se $b \neq 0$, tomemos $K(x, y)=\left(x, \frac{1}{b} y\right)$, assim

$$
K \circ \widetilde{h}:\left\{\begin{array}{l}
x \longmapsto(x, 0) \\
\bar{x} \longmapsto\left(\bar{x}, \bar{x}^{k+1}\right)
\end{array}\right.
$$

Definindo $g=K \circ \widetilde{h}$, temos que ao aplicarmos a Transversal Completa (4.1.4) obtemos que os elementos em $J^{r+1}(1,2) \oplus J^{r+1}(1,2)$ cujo $r$-jato é $g$ são $\mathcal{A}^{r+1}$ equivalentes a $g, r \geq k+1$. Sejam

$$
\begin{aligned}
T \mathcal{K}_{e} \cdot g= & \left\{\begin{array}{cc}
\xi & 0 \\
0 & (k+1) \zeta \bar{x}^{k}
\end{array}\right)+\left(\begin{array}{cc}
\delta \eta_{1}(x, 0) & \gamma \eta_{1}\left(\bar{x}, \bar{x}^{k+1}\right) \\
\delta \eta_{2}(x, 0) & \gamma \eta_{2}\left(\bar{x}, \bar{x}^{k+1}\right)
\end{array}\right), \\
& \left.\xi, \zeta, \delta, \gamma \in \varepsilon_{1} \text { e } \eta_{1}, \eta_{2} \in m_{2}\right\}
\end{aligned}
$$

e

$$
\begin{aligned}
T \mathcal{A}_{e} \cdot g= & \left\{\begin{array}{cc}
\xi & 0 \\
0 & (k+1) \zeta \bar{x}^{k}
\end{array}\right)+\left(\begin{array}{cc}
\eta_{1}(x, 0) & \eta_{1}\left(\bar{x}, \bar{x}^{k+1}\right) \\
\eta_{2}(x, 0) & \eta_{2}\left(\bar{x}, \bar{x}^{k+1}\right)
\end{array}\right), \\
\left.\xi, \zeta \in \varepsilon_{1} \text { e } \eta_{1}, \eta_{2} \in \varepsilon_{2}\right\} &
\end{aligned}
$$


Assim $m_{1}^{k+1} \cdot \varepsilon_{1,2} \oplus m_{1}^{k+1} \cdot \varepsilon_{1,2} \subset T \mathcal{K}_{e} \cdot g$ e $m_{1}^{k} \cdot \varepsilon_{1,2} \oplus m_{1}^{k} \cdot \varepsilon_{1,2} \subset T \mathcal{A}_{e} \cdot g$, pelo Teorema 3.1.5 temos que $g$ é $(2 k+1)$ - $\mathcal{A}$-determinado. Portanto $f$ é $\mathcal{A}$-equivalente a $g$.

Com isto concluímos a classificação dos bigermes cujo os ramos têm corank zero.

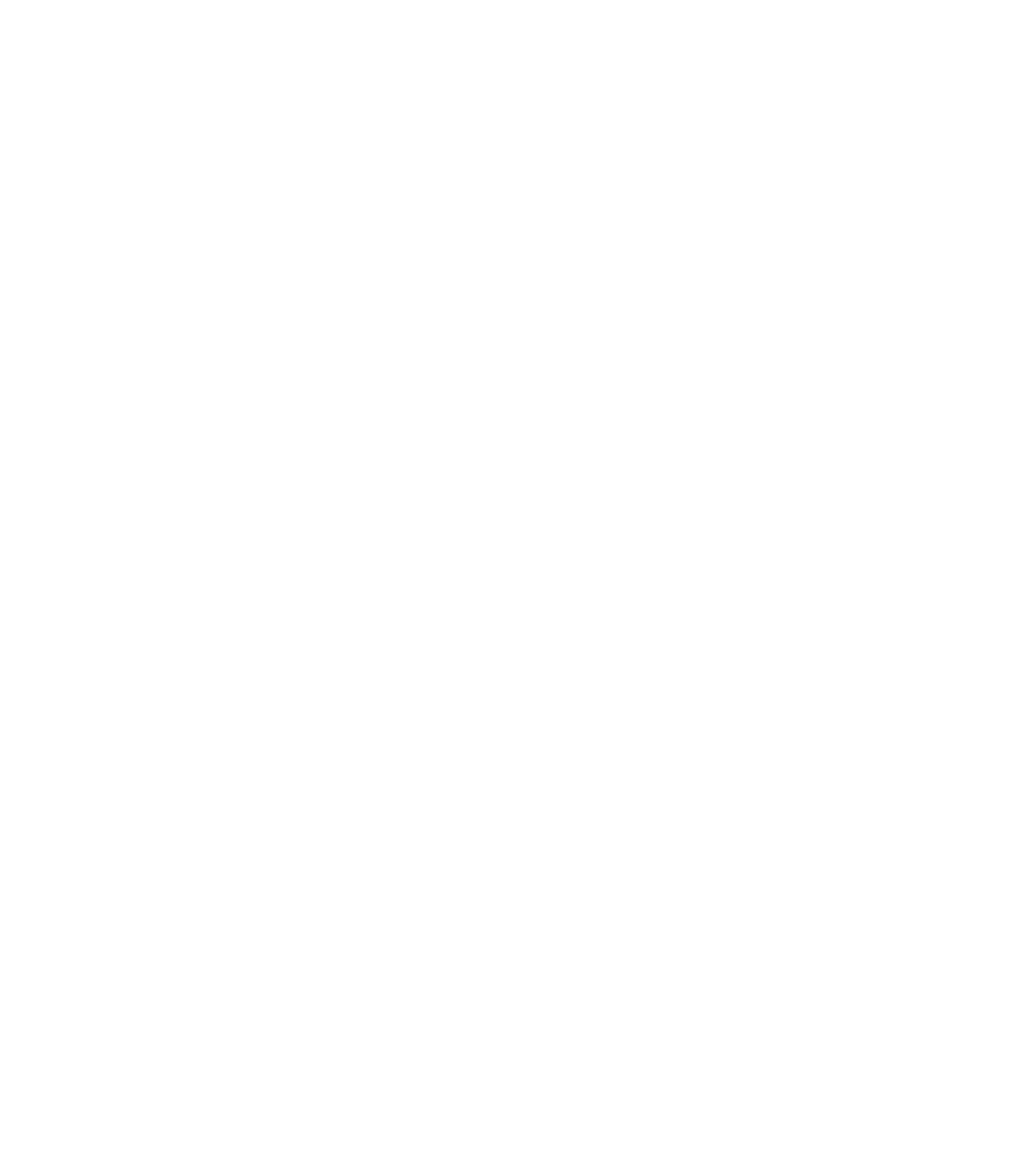


- Suponhamos que os corank de $f_{1}$ e $f_{2}$ sejam 0 e 1 respectivamente. Neste caso temos que $f_{1}$ é uma imersão, então pela Forma Local das Imersões (1.1.4) temos que $f$ é $\mathcal{A}$-equivalente a um bigerme cujo 1-jato é

$$
g:\left\{\begin{array}{l}
x \longmapsto(x, 0) \\
\bar{x} \longmapsto(0,0)
\end{array}\right.
$$

Observemos que $g$ tem $\mathcal{A}$-codimensão infinita, pois

$$
\left(\begin{array}{cc}
0 & 0 \\
0 & \bar{x}^{k}
\end{array}\right) \notin T \mathcal{A} \cdot g=\left\{\left(\begin{array}{cc}
\xi & 0 \\
0 & 0
\end{array}\right)+\left(\begin{array}{cc}
\eta_{1}(x, 0) & 0 \\
\eta_{2}(x, 0) & 0
\end{array}\right), \xi \in m_{1} \text { e } \eta_{1}, \eta_{2} \in m_{2}\right\}
$$

com $k \geq 1$. Apliquemos a Transversal Completa (4.1.4) em $J^{2}(1,2) \oplus J^{2}(1,2)$, encontramos os elementos cujo 1 -jato é $g$ são $\mathcal{A}^{2}$-equivalentes a

$$
h:\left\{\begin{array}{l}
x \stackrel{h_{1}}{\longmapsto}(x, 0) \\
\bar{x} \stackrel{h_{2}}{\longmapsto}\left(\alpha \bar{x}^{2}, \beta \bar{x}^{2}\right)
\end{array}\right.
$$

- Se $\alpha=0$ e $\beta \neq 0$. Tomando $K(x, y)=\left(x, \frac{1}{\beta} y\right)$, temos que

$$
\widetilde{h}=K \circ h:\left\{\begin{array}{l}
x \stackrel{\widetilde{h}_{1}}{\longmapsto}(x, 0) \\
\bar{x} \stackrel{\widetilde{h}_{2}}{\longmapsto}\left(0, \bar{x}^{2}\right)
\end{array}\right.
$$

Ao aplicarmos a Transversal Completa (4.1.4) obtemos que os elementos em $J^{3}(1,2) \oplus J^{3}(1,2)$ cujo 2-jato é $\widetilde{h}$ são $\mathcal{A}^{3}$-equivalentes a

$$
\widetilde{g}:\left\{\begin{array}{l}
x \stackrel{\widetilde{g}_{1}}{\longmapsto}(x, 0) \\
\bar{x} \stackrel{\widetilde{g}_{2}}{\longmapsto}\left(c \bar{x}^{3}, \bar{x}^{2}\right)
\end{array}\right.
$$

Se $c \neq 0$, tomando $H(x)=c x$ e $K(x, y)=\left(\frac{1}{c} x, y\right)$ temos que $K \circ \widetilde{g}_{1} \circ$ $H(x)=(x, 0)$ e $K \circ \widetilde{g}_{2}(\bar{x})=\left(\bar{x}^{3}, \bar{x}^{2}\right) \operatorname{logo} \widetilde{g}$ é $\mathcal{A}^{3}$-equivalente a

$$
\tilde{f}: \begin{cases}x \longmapsto & (x, 0) \\ \bar{x} \longmapsto\left(\bar{x}^{3}, \bar{x}^{2}\right)\end{cases}
$$

E mais, ao aplicarmos a Transversal Completa (4.1.4) em $J^{k}(1,2) \underset{\widetilde{f}}{\oplus}$ $J^{k}(1,2)$, temos que os elementos, cujo 3 -jato é $\widetilde{f}$, são $\mathcal{A}^{k}$-equivalentes a $\widetilde{f}$, $k \geq 4$. 
Sejam

$$
\begin{aligned}
T \mathcal{K}_{e} \cdot \tilde{f}= & \left\{\left(\begin{array}{cc}
\xi & 3 \zeta \bar{x}^{2} \\
0 & 2 \zeta \bar{x}
\end{array}\right)+\left(\begin{array}{cc}
\delta \eta_{1}(x, 0) & \gamma \eta_{1}\left(\bar{x}^{3}, \bar{x}^{2}\right) \\
\delta \eta_{2}(x, 0) & \gamma \eta_{2}\left(\bar{x}^{3}, \bar{x}^{2}\right)
\end{array}\right),\right. \\
& \left.\xi, \zeta, \delta, \gamma \in \varepsilon_{1} \text { e } \eta_{1}, \eta_{2} \in m_{2}\right\}
\end{aligned}
$$

$\mathrm{e}$

$T \mathcal{A}_{e} \cdot \tilde{f}=\left\{\left(\begin{array}{cc}\xi & 3 \zeta \bar{x}^{2} \\ 0 & 2 \zeta \bar{x}\end{array}\right)+\left(\begin{array}{ll}\eta_{1}(x, 0) & \eta_{1}\left(\bar{x}^{3}, \bar{x}^{2}\right) \\ \eta_{2}(x, 0) & \eta_{2}\left(\bar{x}^{3}, \bar{x}^{2}\right)\end{array}\right), \xi, \zeta \in \varepsilon_{1}\right.$ e $\left.\eta_{1}, \eta_{2} \in \varepsilon_{2}\right\}$

Assim $m_{1}^{2} \cdot \varepsilon_{1,2} \oplus m_{1}^{2} \cdot \varepsilon_{1,2} \subset T \mathcal{K}_{e} \cdot \tilde{f}$ e $m_{1}^{2} \cdot \varepsilon_{1,2} \oplus m_{1}^{2} \cdot \varepsilon_{1,2} \subset T \mathcal{A}_{e} \cdot \tilde{f}$, pelo Teorema 3.1.5 temos que $\tilde{f}$ é 4 - $\mathcal{A}$-determinado. Portanto $f$ é $\mathcal{A}$-equivalente a $\tilde{f}$.

Mas se $c=0$ temos que

$$
\widetilde{g}:\left\{\begin{array}{l}
x \stackrel{\widetilde{g}_{1}}{\longmapsto}(x, 0) \\
\bar{x} \stackrel{\widetilde{g}_{2}}{\longmapsto}\left(0, \bar{x}^{2}\right)
\end{array}\right.
$$

Aplicando a Transversal Completa temos que os elementos em $J^{k}(1,2) \oplus$ $J^{k}(1,2)$ cujo $(k-1)$-jato é $\widetilde{g}$ são $\mathcal{A}^{k}$-equivalentes a $\widetilde{g}$, se $k$ for par ou são $\mathcal{A}^{k}$-equivalentes a

$$
\widetilde{h}:\left\{\begin{array}{l}
x \stackrel{\widetilde{g}_{1}}{\longmapsto}(x, 0) \\
\bar{x} \stackrel{\widetilde{g}_{2}}{\longmapsto}\left(d \bar{x}^{k}, \bar{x}^{2}\right)
\end{array}\right.
$$

se $k$ for ímpar, com $k \geq 3$.

Se $d \neq 0, \widetilde{h}$ é $\mathcal{A}$-equivalente a

$$
h:\left\{\begin{array}{l}
x \longmapsto(x, 0) \\
\bar{x} \longmapsto\left(\bar{x}^{k}, \bar{x}^{2}\right)
\end{array}\right.
$$

Ao aplicarmos a Transversal Completa (4.1.4) obtemos que os elementos em $J^{r+1}(1,2) \oplus J^{r+1}(1,2)$, cujo $r$-jato é $h$, são $\mathcal{A}^{r+1}$-equivalentes a $h, r \geq k$. Sejam

$$
\begin{aligned}
& T \mathcal{K}_{e} \cdot h=\left\{\left(\begin{array}{cc}
\xi & k \zeta \bar{x}^{k-1} \\
0 & 2 \zeta \bar{x}
\end{array}\right)+\left(\begin{array}{cc}
\delta \eta_{1}(x, 0) & \gamma \eta_{1}\left(\bar{x}^{k}, \bar{x}^{2}\right) \\
\delta \eta_{2}(x, 0) & \gamma \eta_{2}\left(\bar{x}^{k}, \bar{x}^{2}\right)
\end{array}\right)\right. \\
& \left.\xi, \zeta, \delta, \gamma \in \varepsilon_{1} \text { e } \eta_{1}, \eta_{2} \in m_{2}\right\}
\end{aligned}
$$


e

$$
\begin{aligned}
T \mathcal{A}_{e} \cdot h= & \left\{\left(\begin{array}{cc}
\xi & k \zeta \bar{x}^{k-1} \\
0 & 2 \zeta \bar{x}
\end{array}\right)+\left(\begin{array}{cc}
\eta_{1}(x, 0) & \eta_{1}\left(\bar{x}^{k}, \bar{x}^{2}\right) \\
\eta_{2}(x, 0) & \eta_{2}\left(\bar{x}^{k}, \bar{x}^{2}\right)
\end{array}\right),\right. \\
& \left.\xi, \zeta \in \varepsilon_{1} \text { e } \eta_{1}, \eta_{2} \in \varepsilon_{2}\right\}
\end{aligned}
$$

Assim $m_{1}^{k} \cdot \varepsilon_{1,2} \oplus m_{1}^{k} \cdot \varepsilon_{1,2} \subset T \mathcal{K}_{e} \cdot h$ e $m_{1}^{k} \cdot \varepsilon_{1,2} \oplus m_{1}^{k} \cdot \varepsilon_{1,2} \subset T \mathcal{A}_{e} \cdot h$, pelo Teorema 3.1.5 temos que $h$ é $2 k$ - $\mathcal{A}$-determinado. Portanto $f$ é $\mathcal{A}$-equivalente a $h$. 


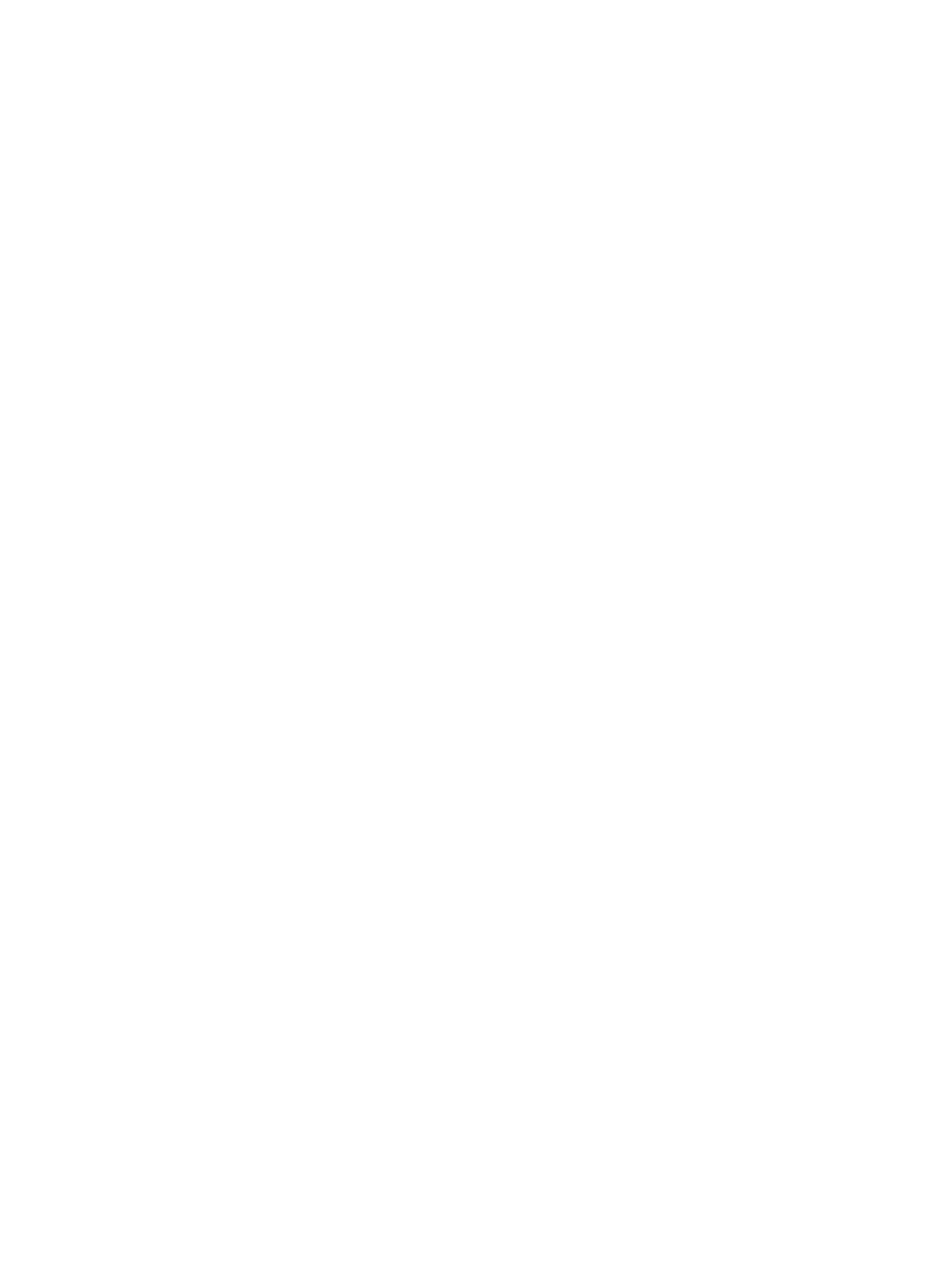


- Se $\alpha \neq 0$ e $\beta=0$. Temos que $h$ é $\mathcal{A}$-equivalente a

$$
\widetilde{h}:\left\{\begin{array}{l}
x \longmapsto(x, 0) \\
\bar{x} \longmapsto\left(\bar{x}^{2}, 0\right)
\end{array}\right.
$$

com $\mathcal{A}$-codimensão infinita, pois

$$
\begin{aligned}
\left(\begin{array}{cc}
0 & 0 \\
0 & \bar{x}^{2 k+1}
\end{array}\right) \notin T \mathcal{A} \cdot \widetilde{h}= & \left\{\left(\begin{array}{cc}
\xi & 2 \zeta \bar{x} \\
0 & 0
\end{array}\right)+\left(\begin{array}{cc}
\eta_{1}(x, 0) & \eta_{1}\left(\bar{x}^{2}, 0\right) \\
\eta_{2}(x, 0) & \eta_{2}\left(\bar{x}^{2}, 0\right)
\end{array}\right),\right. \\
& \left.\xi, \zeta \in m_{1} \text { e } \eta_{1}, \eta_{2} \in m_{2}\right\}
\end{aligned}
$$

com $k \geq 1$.

Aplicando a Transversal Completa (4.1.4) obtemos que estes elementos em $J^{k}(1,2) \oplus J^{k}(1,2)$, cujo $(k-1)$-jato é $\widetilde{h}$, são $\mathcal{A}^{k}$-equivalentes a

$$
\widetilde{g}:\left\{\begin{array}{l}
x \longmapsto(x, 0) \\
\bar{x} \longmapsto\left(\bar{x}^{2}, d \bar{x}^{k}\right)
\end{array}\right.
$$

com $k \geq 3$. Temos que se $d \neq 0, \widetilde{g}$ é $\mathcal{A}$-equivalente a

$$
g:\left\{\begin{array}{l}
x \longmapsto(x, 0) \\
\bar{x} \longmapsto\left(\bar{x}^{2}, \bar{x}^{k}\right)
\end{array}\right.
$$

I) Se $k$ for ímpar. Ao aplicarmos a Transversal Completa (4.1.4) temos que os elementos em $J^{r+1}(1,2) \oplus J^{r+1}(1,2)$, cujo $r$-jato é $g$, são $\mathcal{A}^{r+1}$-equivalentes a $g, \operatorname{com} r \geq k$.

Sejam

$$
\begin{aligned}
T \mathcal{K}_{e} \cdot g= & \left\{\begin{array}{cc}
\xi & 2 \zeta \bar{x} \\
0 & k \zeta \bar{x}^{k-1}
\end{array}\right)+\left(\begin{array}{cc}
\delta \eta_{1}(x, 0) & \gamma \eta_{1}\left(\bar{x}^{2}, \bar{x}^{k}\right) \\
\delta \eta_{2}(x, 0) & \gamma \eta_{2}\left(\bar{x}^{2}, \bar{x}^{k}\right)
\end{array}\right), \\
& \left.\xi, \zeta, \delta, \gamma \in \varepsilon_{1} \text { e } \eta_{1}, \eta_{2} \in m_{2}\right\}
\end{aligned}
$$

e

$$
\begin{aligned}
T \mathcal{A}_{e} \cdot g= & \left\{\begin{array}{cc}
\xi & 2 \zeta \bar{x} \\
0 & k \zeta \bar{x}^{k-1}
\end{array}\right)+\left(\begin{array}{cc}
\eta_{1}(x, 0) & \eta_{1}\left(\bar{x}^{2}, \bar{x}^{k}\right) \\
\eta_{2}(x, 0) & \eta_{2}\left(\bar{x}^{2}, \bar{x}^{k}\right)
\end{array}\right), \\
& \left.\xi, \zeta \in \varepsilon_{1} \text { e } \eta_{1}, \eta_{2} \in \varepsilon_{2}\right\}
\end{aligned}
$$

Assim $m_{1}^{k} \cdot \varepsilon_{1,2} \oplus m_{1}^{k} \cdot \varepsilon_{1,2} \subset T \mathcal{K}_{e} \cdot g$ e $m_{1}^{k} \cdot \varepsilon_{1,2} \oplus m_{1}^{k} \cdot \varepsilon_{1,2} \subset T \mathcal{A}_{e} \cdot g$, pelo Teorema 3.1.5 temos que $h$ é $2 k-\mathcal{A}$-determinado. Portanto $f$ é $\mathcal{A}$-equivalente a $g$. 
II) Se $k$ for par. Ao aplicarmos a Transversal Completa (4.1.4) temos que os elementos em $J^{r+1}(1,2) \oplus J^{r+1}(1,2)$, cujo $r$-jato é $g$, são $\mathcal{A}^{r+1}$-equivalentes a $g$, se $r$ for ímpar ou são $\mathcal{A}^{r+1}$-equivalentes a

$$
\widetilde{g}:\left\{\begin{array}{l}
x \mapsto(x, 0) \\
\bar{x} \mapsto\left(\bar{x}^{2}, \bar{x}^{k}+d \bar{x}^{r+1}\right)
\end{array}\right.
$$

se $r$ for par, com $r \geq k$.

Se $d \neq 0$, temos que $\widetilde{g}$ é $\mathcal{A}$-equivalente a

$$
h:\left\{\begin{array}{l}
x \mapsto(x, 0) \\
\bar{x} \mapsto\left(\bar{x}^{2}, \bar{x}^{k}+\bar{x}^{r+1}\right)
\end{array}\right.
$$

Ao aplicarmos a Transversal Completa (4.1.4) temos que os elementos em em $J^{l+1}(1,2) \oplus J^{l+1}(1,2)$, cujo $l$-jato é $h$, são $\mathcal{A}^{l+1}$-equivalentes a $h, l \geq r+1$.

Sejam

$$
T \mathcal{K}_{e} \cdot h=\left\{\begin{array}{cc}
\left(\begin{array}{cc}
\xi & 2 \zeta \bar{x} \\
0 & \zeta\left[k \bar{x}^{k-1}+(r+1) \bar{x}^{r}\right]
\end{array}\right)+\left(\begin{array}{cc}
\delta \eta_{1}(x, 0) & \gamma \eta_{1}\left(\bar{x}^{2}, \bar{x}^{k}+\bar{x}^{r+1}\right) \\
\delta \eta_{2}(x, 0) & \gamma \eta_{2}\left(\bar{x}^{2}, \bar{x}^{k}+\bar{x}^{r+1}\right)
\end{array}\right), \\
\left.\xi, \zeta, \delta, \gamma \in \varepsilon_{1} \text { e } \eta_{1}, \eta_{2} \in m_{2}\right\}
\end{array}\right.
$$

e

$$
T \mathcal{A}_{e} \cdot h=\left\{\begin{array}{cc}
\left(\begin{array}{cc}
\xi & 2 \zeta \bar{x} \\
0 & \zeta\left[k \bar{x}^{k-1}+(r+1) \bar{x}^{r}\right]
\end{array}\right)+\left(\begin{array}{cc}
\eta_{1}(x, 0) & \eta_{1}\left(\bar{x}^{2}, \bar{x}^{k}+\bar{x}^{r+1}\right) \\
\eta_{2}(x, 0) & \eta_{2}\left(\bar{x}^{2}, \bar{x}^{k}+\bar{x}^{r+1}\right)
\end{array}\right), \\
\left.\xi, \zeta \in \varepsilon_{1} \mathrm{e} \eta_{1}, \eta_{2} \in \varepsilon_{2}\right\}
\end{array}\right.
$$

Assim $m_{1}^{r} \cdot \varepsilon_{1,2} \oplus m_{1}^{r} \cdot \varepsilon_{1,2} \subset T \mathcal{K}_{e} \cdot h$ e $m_{1}^{r+1} \cdot \varepsilon_{1,2} \oplus m_{1}^{r+1} \cdot \varepsilon_{1,2} \subset T \mathcal{A}_{e} \cdot h$, pelo Teorema 3.1.5 temos que $h$ é $2 r+1$ - $\mathcal{A}$-determinado. Portanto $f$ é $\mathcal{A}$ equivalente a $g$. 


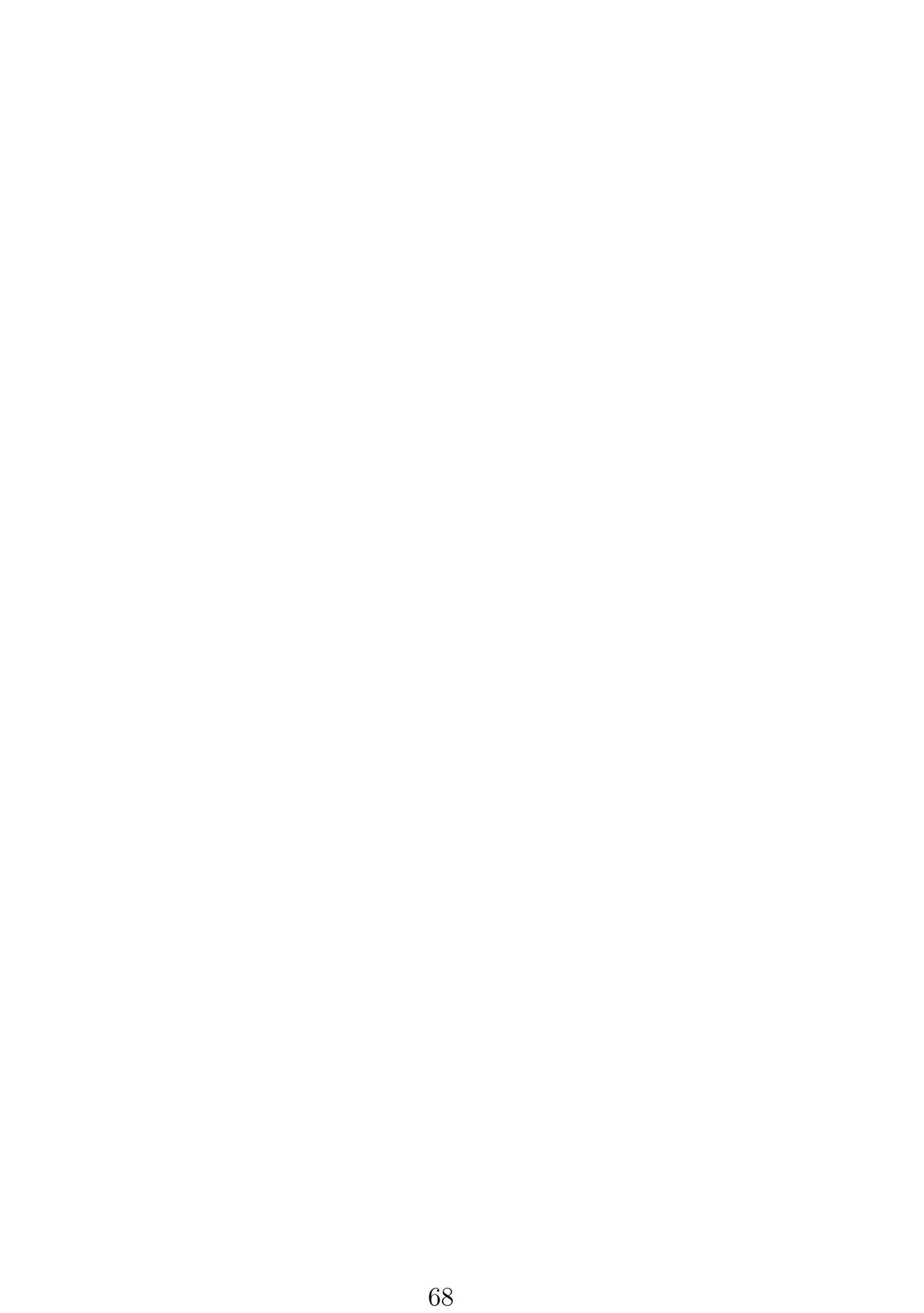




\section{Referências Bibliográficas}

[1] V.I. Arnold, Normal forms for functions near degenerate critical points, the Weyl groups of $A_{k}, D_{k}, E_{k}$ and Lagrangian Singularities, Funct. Anal. and its Appl. 6 (1972), 254-272.

[2] J.W. Bruce, Classification Problems in Singularity Theory, Notas de mini-curso apresentado no 5th Workshop on Real and Complex Singularities, ICMC-USP, São Carlos, SP, 1998.

[3] J.B. Conway, Functions of one complex variable, Graduate Text in Mathematics, vol. 11, Springer-Verlag, N.Y. 1978.

[4] C.G. Gibson, Singular points of smooth mappings. Research Notes in Maths. 25, Pitman, London 1973.

[5] M. Golubitsky and V. Guillemin, Stable mappings and their singularities. Graduate Text in Mathematics, vol. 14, Springer-Verlag, N.Y. 1973.

[6] V. Guillemin and A. Pollack, Differential Topology, Prentice-Hall, Inc., Englewood Cliffs, New Jersey, 1974.

[7] E.L. Lima, Álgebra Linear. Coleção Matemática Universitária, IMPA, 1998.

[8] C. Hobs e N. Kirk, On the classification and bifurcation of multigerms of maps from surfaces to 3-space, Math Scand 89 (2001), nº. 1, 57-96.

[9] F. Tari, Singularidades de aplicações difernciáveis, Notas didáticas do ICMC, $n^{o}$. 34, ICMC - USP - São Carlos, 1999.

[10] Neil P. Kirk, Transversal - A Maple Package For Singularity Theory, Version 3.1, November 1998. (Obs.: Este é um "software"). 
[11] M. Reid, Undergraduate Algebraic Geometry, London Mathematical Society, Student Texts 12, Cambridge University Press, 1988. 